\title{
Lipid peroxidation and antioxidants in humans : effects of oxidative stress and dietary n-3 fatty acids
}

Citation for published version (APA):

Oostenbrug, G. S. (1996). Lipid peroxidation and antioxidants in humans : effects of oxidative stress and dietary $n-3$ fatty acids. [Doctoral Thesis, Maastricht University]. Maastricht University. https://doi.org/10.26481/dis.19961219go

Document status and date:

Published: 01/01/1996

DOI:

10.26481/dis.19961219go

Document Version:

Publisher's PDF, also known as Version of record

\section{Please check the document version of this publication:}

- A submitted manuscript is the version of the article upon submission and before peer-review. There can be important differences between the submitted version and the official published version of record.

People interested in the research are advised to contact the author for the final version of the publication, or visit the DOI to the publisher's website.

- The final author version and the galley proof are versions of the publication after peer review.

- The final published version features the final layout of the paper including the volume, issue and page numbers.

Link to publication

\footnotetext{
General rights rights.

- You may freely distribute the URL identifying the publication in the public portal. please follow below link for the End User Agreement:

www.umlib.nl/taverne-license

Take down policy

If you believe that this document breaches copyright please contact us at:

repository@maastrichtuniversity.nl

providing details and we will investigate your claim.
}

Copyright and moral rights for the publications made accessible in the public portal are retained by the authors and/or other copyright owners and it is a condition of accessing publications that users recognise and abide by the legal requirements associated with these

- Users may download and print one copy of any publication from the public portal for the purpose of private study or research.

- You may not further distribute the material or use it for any profit-making activity or commercial gain

If the publication is distributed under the terms of Article $25 \mathrm{fa}$ of the Dutch Copyright Act, indicated by the "Taverne" license above, 


\section{Lipid peroxidation and antioxidants in humans}

EFFECTS OF OXIDATIVE STRESS AND DIETARY N-3 FATTY ACIDS 
Oostenbrug, Gerard Simon

Lipid peroxidation and antioxidants in humans. Effects of oxidative stress and dietary $n-3$ fatty acids / Gerard Simon Oostenbrug Thesis Maastricht University, Maastricht, The Netherlands - with summary in Dutch. ISBN 90-9009971-9

(C) 1996 Oostenbrug, Maastricht, The Netherlands 


\title{
Lipid peroxidation and antioxidants in humans
} EFFECTS OF OXIDATIVE STRESS AND DIETARY N-3 FATTY ACIDS

\section{PROEFSCHRIFT}

\author{
ter verkrijging van de graad van doctor \\ aan de Universiteit Maastricht, \\ op gezag van de Rector Magnificus, prof. mr. M.J. Cohen \\ volgens het besluit van het College van Dekanen, \\ in het openbaar to verdedigen \\ op donderdag 19 december 1996 om 16.00 uur
}

door

\section{Gerard Simon Oostenbrug}

geboren te Deventer op 18 februari 1962 
Promotor:

Co-promotor:

Beoordelingscommissie: Prof. dr. J.C.S. Kleinjans (voorzitter)

Prof. dr. C.E. Blanco

Dr. F. Brouns

Prof. dr. A.T. Diplock (University of London, United Kingdom)

Prof. dr. F.A. Huf (Technische Universiteit Eindhoven)

Financial support by the Netherlands Heart Foundation and the Foundation dr. ir. J.H.J. van de Laar for the publication of this thesis is gratefully acknowledged. 
Aan mijn ouders 



\section{CONTENTS}

Chapter 1

Chapter 2

Chapter 3

Chapter 4

Chapter 5

Chapter 6

Chapter 7

Samenvatting

General discussion lipoprotein in vitro supplementation stress in humans
Effects of fish oil and vitamin E supplementation on copper-catalyzed oxidation of human low density

Physical performance, red blood cell characteristics, plasma lipid-soluble antioxidant concentrations and in vitro oxidation of low density lipoproteins during exercise. The effects of fish oil and vitamin $E$

Maternal and neonatal plasma lipid-soluble antioxidant levels in normal pregnancy, and the relationship with fatty acid unsaturation

Pregnancy complicated with pregnancy-induced hypertension: maternal and neonatal plasma lipidsoluble antioxidant levels and its relationship with fatty acid unsaturation

Percutaneous transluminal coronary angioplasty as model for lipid peroxidation-associated oxidative

Dankwoord

Curriculum vitae 



\section{GENERAL INTRODUCTION}

\section{Oxygen free radicals}

Oxygen free radicals are oxygen species with one or more unpaired electrons, which are involved in many normal biological processes, like phagocytosis, eicosanoid metabolism and enzymatic reactions. Antioxidants and antioxidant enzymes inhibit the formation of radicals or reduce their reactivity ${ }^{1}$. However, any situation accompanied by increased oxidative stress and/or decreased antioxidant defences, may lead to an excess of free radicals and damage to biomolecules. In various clinical conditions, involvement of oxygen free radicals has been suggested (reviewed in ref. 2). Halliwell and Gutteridge ${ }^{2}$ pointed out, however, that the production of free radicals is usually a consequence of disease. On the other hand, evidence has accumulated that oxygen free radicals are involved in disease pathology of atherosclerosis and ischemia/reperfusion injury? ${ }^{2}$.

Due to their short half-lives, relatively low concentrations, and the ethical and logistical considerations in obtaining samples, however, the detection of free radicals in human tissue with spin labels is usually not feasible ${ }^{3}$. Also, it should be realized that production of free radicals does not mean that biomolecules will actually be damaged.

\section{Lipid peroxidation}

Several oxygen free radicals are capable of initiating lipid peroxidation by removing a hydrogen atom from a methylene group of an unsaturated fatty acid ${ }^{4}$ (Figure 1.1). Also non-radical reactive oxygen species, like hydrogen peroxide, may play a role in lipid peroxidation ${ }^{1}$. Polyunsaturated fatty acids (PUFA) in membranes are a major target of reactive oxygen species, since the presence of multiple double bonds facilitates abstraction of a hydrogen atom. In vitro studies have shown that after hydrogen abstraction, the PUFA radicals undergo molecular rearrangement to form conjugated dienes, and then take up oxygen to form peroxyl radicals ${ }^{4}$ (for an extensive review of oxygen radical chemistry of PUFA, see also ref. 5). Peroxyl radicals can 
attack membrane proteins, but are also capable of abstracting a hydrogen atom from another PUFA, and start a chain reaction while the peroxyl radical is converted to a hydroperoxide. This chain reaction may be terminated by a chain-breaking antioxidant, like vitamin $\mathrm{E}^{4}$. Peroxyl radicals may also be broken down to cyclic (endo)peroxides when the presence of good hydrogen donators, like vitamin E or PUFA themselves, is low $w^{5}$. Lipid hydroperoxides are fairly stable molecules under physiological conditions, but their decomposition is catalyzed by transition metals and metal complexes ${ }^{1,4}$. The subsequently formed alkoxyl and peroxyl radicals can re-initiate PUFA peroxidation, and amplify the initial events ${ }^{1}$. Various end-products arise from breakdown of lipid hydroperoxides and endoperoxides, like alkanes, alkenes, alkanals, and alkenals.

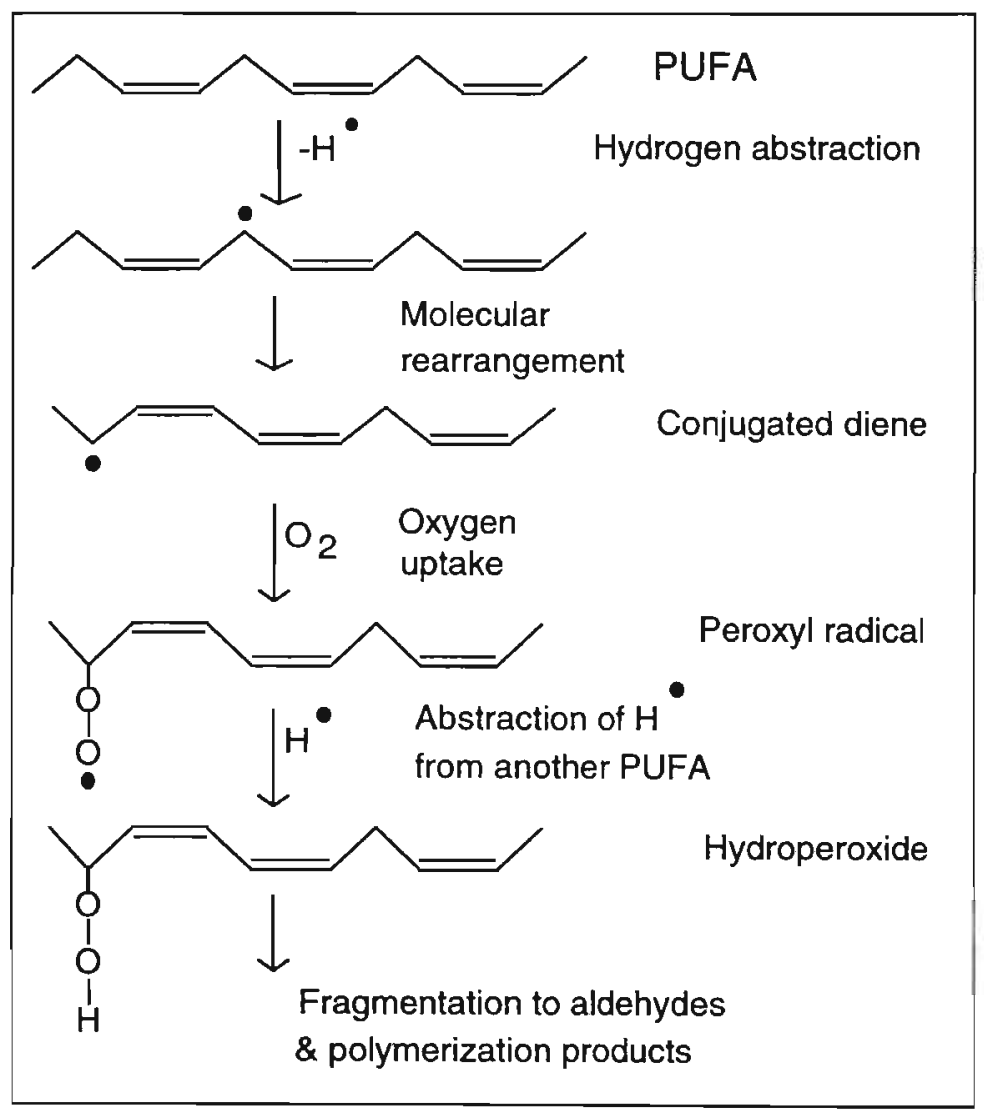

Figure 1.1. Initiation and propagation of peroxidation of a polyunsaturated fatty acid (PUFA) with three double bonds. 
The formation of many of these products has been used to monitor lipid peroxidation ${ }^{6}$. However, some of these indices can be non-specific, and their production may not necessarily be directly related to lipid peroxidation ${ }^{4}$. For example, alkanes like ethane (from n-3 fatty acids) and pentane (from n-6 fatty acids) are only minor end-products and their formation depends on the presence of transition metals to decompose lipid peroxides ${ }^{4}$. Furthermore, these alkanes may arise through flushing of adipose tissue, and pentane may be metabolized by the liver before its detection in breath ${ }^{7}$. A frequently used method to quantify the degree of lipid peroxidation is the detection of malondialdehyde (MDA) by the reaction with thiobarbituric acid (TBA). MDA is usually formed from fatty acids with three or more double bonds, and the amount of free MDA produced in most peroxidizing lipid systems would not give a significant color yield in the TBA-test. The majority of the MDA detected in the TBA test is formed from decomposition of lipid peroxides and further peroxidation during the acid-heating stage of the test itself ${ }^{2,8}$. In addition the TBA test is not specific for $M A^{8}$. However, as yet no ideal method exists to assess lipid peroxidation in humans, and results need to be interpreted with caution.

\section{Peroxidation of low density lipoproteins}

Lipid peroxidation is not only limited to cellular membranes, but also takes place in, for example, lipoproteins. Steinberg et al. ${ }^{9}$ have suggested that part of the etiology of atherosclerosis may be explained by lipid peroxidation of low density lipoproteins (LDL). This hypothesis, which has been supported by several other studies in recent years, is depicted in Figure 1.2. Briefly, LDL in the intima of vessel walls, may be oxidized when (1) oxidized lipids from within cells are transferred to LDL, (2) LDL lipids are directly oxidized by LDL-cell contact, or when (3) reactive oxygen species are generated within the cell and released into the medium, where oxidation of LDL lipids occurs. Oxidized LDL then (Figure 1.2, I) increases the recruitment of circulating monocytes, (II) inhibits the motility of resident macrophages to leave the intima, and (III) the oxidized LDL is taken up more rapidly by macrophages, leading to the formation of foam cells. In addition, oxidized LDL has a direct cytotoxic effect (IV), leading to loss of endothelial integrity. 
Esterbauer et al. ${ }^{10}$ developed a technique to monitor the susceptibility of LDL to oxidation in vitro. Briefly, LDL is isolated from plasma, and a fixed concentration of LDL is oxidized with copper ions. Although the mechanism of copper-catalyzed oxidation is not yet completely elucidated, copper probably generates radicals capable of propagating lipid peroxidation by decomposing lipid hydroperoxides which are also present in native LDL. The formation of conjugated dienes is monitored spectrophotometrically at $234 \mathrm{~nm}$ (Figure 1.3). After addition of copper, antioxidants contained in LDL are consumed, and during this lag phase the increase in conjugated dienes is minimal. The lag phase is followed by the propagation phase, characterized by rapid oxidation of polyunsaturated fatty acids. The rapid increase in conjugated dienes reaches a maximum, after which the absorbance at $234 \mathrm{~nm}$ decreases again, due to breakdown of the conjugated dienes.

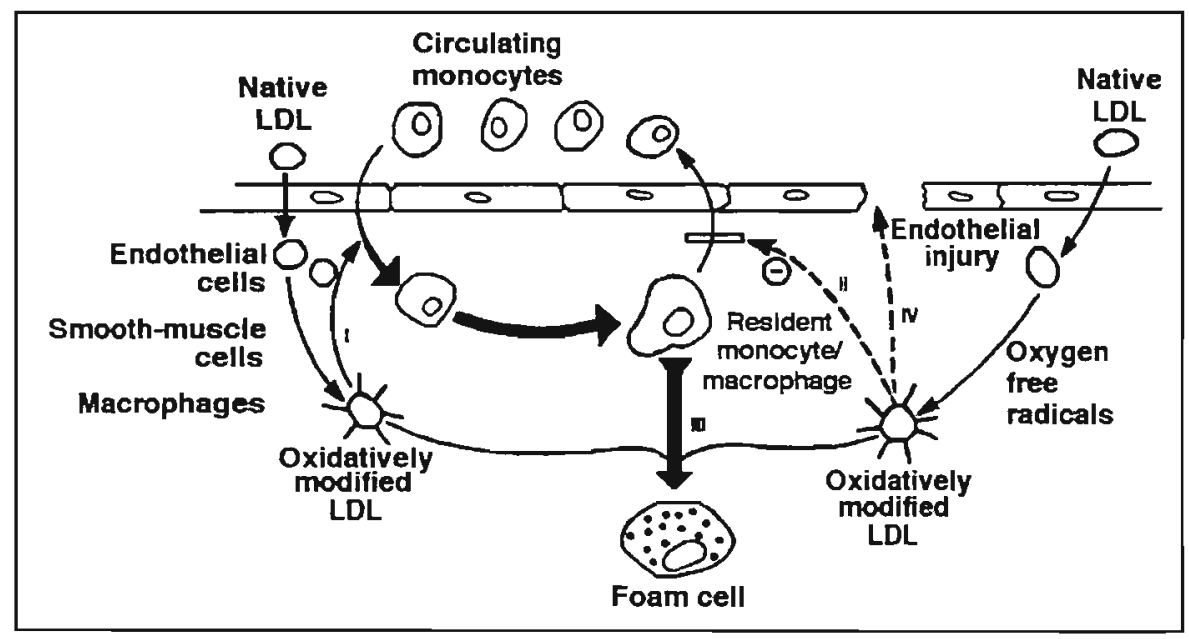

Figure 1.2. Four mechanisms by which oxidation of LDL may contribute to atherosclerosis (see text). [Reprinted by permission of The New England Journal of Medicine, Steinberg et al. ${ }^{9}$, p. 919. Copyright 1989. Massachusetts Medical Society. All rights reserved]

The susceptibility of LDL to oxidation in vitro is studied under conditions where the LDL is not protected by the interacting (water-soluble) antioxidant systems present in the human body, and with copper as prooxidant. Nevertheless, in vitro copper-oxidized LDL greatly resembles LDL extracted from human and rabbit atherosclerotic lesions ${ }^{11}$, and $\operatorname{lgG}$ isolated from these lesions recognizes copper-oxidized LDL but not native $\mathrm{LDL}^{12}$. Moreover, Regnström et al. ${ }^{13}$ showed an inverse relation 
between the lag phase for copper-catalyzed LDL oxidation in vitro and quantitative estimates of global coronary atherosclerosis in humans. This technique may therefore provide a useful tool to assess effects of interventions on LDL oxidizability.

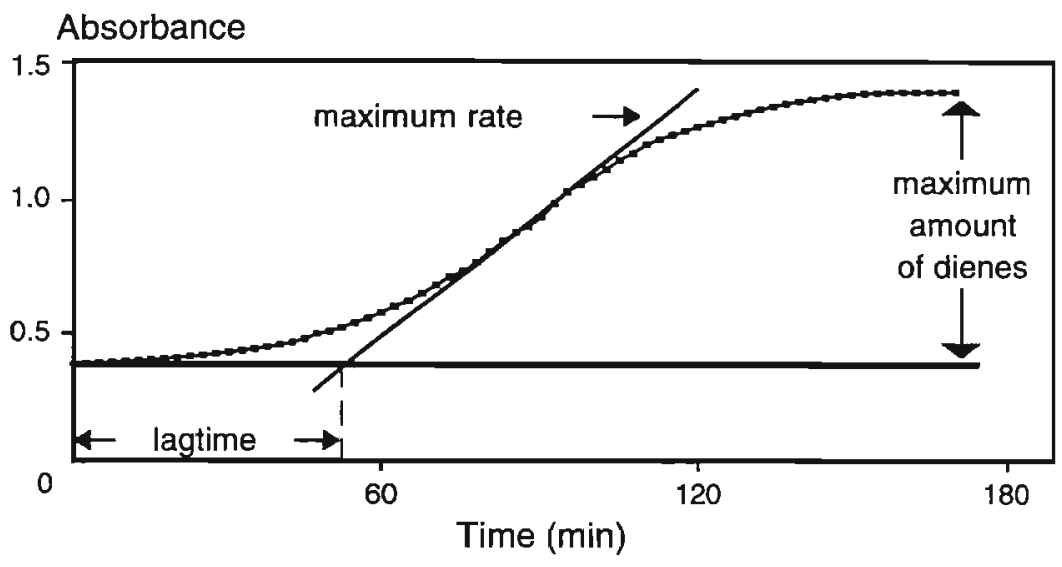

Figure 1.3. Formation of conjugated dienes during copper-catalyzed oxidation of LDL in vitro is monitored spectrophotometrically at $234 \mathrm{~nm}$. The lagtime before onset of rapid oxidation, the maximum rate of oxidation during the propagation phase, and the maximum amount of dienes formed, are used to described LDL oxidation characteristics.

\section{Outline of this thesis}

In situations in which oxidative stress is increased, and/or antioxidative protection is decreased, lipids, proteins, carbohydrates, and DNA may be subjected to oxidative damage. Antioxidative systems, like lipid-soluble antioxidants, antioxidant enzymes, water-soluble antioxidants, and antioxidant proteins may protect biomolecules from oxidative damage. This thesis focusses on the oxidative and antioxidative processes, closely related to lipids, under mainly physiological conditions.

Firstly, the effect of dietary manipulation of fatty acids on lipid peroxidation of LDL was studied (Chapter 2). The hypothesis for atherogenesis by Steinberg of al. ${ }^{9}$ suggests that the susceptibility of LDL to oxidation may be an important factor in atherosclerosis. Since PUFA are highly susceptible to oxidation due to the presence of multiple double bonds, diets were manipulated with fish oil, which contains a high 
degree of PUFA with 5 and 6 double bonds. Cosgrove et al. ${ }^{14}$ showed that the in vitro oxidizability of PUFA is linearly dependent on the number of double bonds, and that the oxidizability of docosahexaenoic acid, with 6 double bonds, was 5 times greater than the oxidizability of linoleic acid, with 2 double bonds. Therefore, incorporation of these highly unsaturated fish oil fatty acids in LDL might increase their susceptibility to oxidation.

In addition, the effects of dietary fish oil during strenuous physical exercise were studied (Chapter 3). Several studies have indicated that dietary fish oils increase the deformability of red blood cells $s^{15,16}$ and facilitate the transport of red blood cells through capillaries ${ }^{17}$, leading to improved oxygen supply and exercise performance. However, during exercise the production of free radicals increases ${ }^{18}$, and, especially in combination with the high degree of unsaturation of fish oils, may increase lipid peroxidation. Therefore, we have studied exercise performance and physical characteristics of red blood cells, as well as copper-catalyzed oxidation of LDL in vitro and plasma lipid-soluble antioxidant levels during intensive exercise.

During pregnancy, concentrations of various lipids increase in plasma. A previous study at our department showed that levels of plasma phospholipid fatty acids, which are rich in highly unsaturated PUFA with three or more double bonds, increased by more than $50 \%$ during pregnancy ${ }^{19}$. We have studied whether the increase in fatty acids, serving as substrate for lipid peroxidation, compromised antioxidant defences (Chapter 4). Also, postpartum maternal and neonatal plasma antioxidant levels were studied, since phospholipid fatty acid patterns were changed after delivery ${ }^{19}$, and the stressful event of labor may be associated with free radical production.

Furthermore, there is considerable evidence that endothelial cell injury is involved in the pathogenesis of pregnancy-induced hypertension $(\mathrm{PIH})^{20}$. Therefore, we investigated whether a hypothesis of free radical and lipid peroxidation associated pathogenesis of PIH was supported by altered antioxidant defences (Chapter 5).

As previously pointed out, oxygen free radicals may also be involved in disease pathology of ischemia/reperfusion injury. Percutaneous transluminal coronary angioplasty (PTCA) is a medical procedure associated with ischemia/reperfusion injury in humans. During this procedure, a balloon catheter is inflated intraarterially to reduce arterial stenosis in the heart. Reperfusion of the previously ischemic part, however, suddenly increases the oxygen supply, and the balance between reactive oxygen species, lipid peroxidation products, and antioxidative systems may become derailed. 
Since PTCA is the most routinely used technique under well standardized surgical conditions, it might provide a good model for oxidative stress-related lipid peroxidation in humans (Chapter 6).

The general conclusions emerging from these studies are discussed in Chapter 7.

\section{REFERENCES}

1. Rice-Evans $C$, Burdon R. Free radical-lipid interactions and their pathological consequences. Prog Lipid Res 1993; 32:71-110.

2. Halliwell $B$, Gutteridge JMC. Role of free radicals and catalytic metal ions in human disease: An overview. Meth Enzymol 1990; 186:1-85.

3. Slater TF Cheeseman KH, Davies MJ, Proudfoot $\mathrm{K}$, Xin W. Free radical mechanisms in relation to tissue injury. Proc Nutr Soc 1987; 46:1-12.

4. Halliwell B, Gutteridge JMC. The importance of free radicals and catalytic metal ions in human diseases. Mol Aspects Med 1985; 8:89-193.

5. Gardner HW. Oxygen radical chemistry of polyunsaturated fatty acids. Free Rad Biol Med 1989; 7:65-86.

6. Pryor WA, Shipley Godber S. Noninvasive measures of oxidative stress status in man. Free Rad Biol Med 1991; 10:177-184.

7. Duthie GG, Arthur JR, Mills CF. Tissue damage in vitamin E deficient rats is not detected by expired ethane and pentane. Free Rad Res Commun 1987; 4:21-25.

8. Janero DR. Malondialdehyde and thiobarbituric acid-reactivity as diagnostic indices of lipid peroxidation and peroxidative tissue injury. Free Rad Biol Med 1990; 9:515-540.

9. Steinberg D, Parthasarathy S, Carew TE, Khoo JC, Witztum JL. Beyond cholesterol. Modifications of low-density lipoprotein that increase its atherogenicity. N Engl J Med 1989; 320:915-924.

10. Esterbauer $H$, Striegl $G$, Puhl $H$, Rotheneder $M$. Continuous monitoring of in vitro oxidation of human low density lipoprotein. Free Rad Res Commun 1989; 6:67-75.

11. Ylā-Herttuala S, Palinski W, Rosenfeld ME, Parthasarathy S, Carew TE, Butler S, Witztum JL, Steinberg D. Evidence for the presence of oxidatively modified low density lipoprotein in atherosclerotic lesions of rabbit and man. $J$ Clin invest 1989; 84:1086-1095.

12. Ylā-Herttuala S, Palinski W, Butler SW, Picard S, Steinberg D, Witztum JL. Rabbit and human atherosclerotic lesions contain IgG that recognizes epitopes of oxidized LDL. Arterioscler Thromb 1994; 14:32-40.

13. Regnstrôm J, Nilsson J, Tornvall P, Landou C, Hamsten A. Susceptibility to low-density lipoprotein oxidation and coronary atherosclerosis in man. Lancet 1992; 339:1183-1186.

14. Cosgrove JP, Church DF, Pryor WA. The kinetics of the autoxidation of polyunsaturated fatty acids. Lipids 1987; 22:299-304.

15. Terano T, Hirai A, Hamazaki T, Kobayashi S, Fujita T, Tamura Y, Kumagai A. Effect of oral administration of highly purified eicosapentaenoic acid on platelel function, blood viscosity and red cell deformability in healthy human subjects. Atherosclerosis 1983; 46:321-331. 
16. Cartwright IJ, Pockley AG, Galloway JH, Greaves M, Preston FE. The effects of dietary w-3 polyunsaturated fatty acids on erythrocyte membrane phospholipids, erythrocyte deformability and blood viscosity in healthy volunteers. Atherosclerosis 1985; 55:267-281.

17. Bruckner G, Webb P, Greenwell L, Chow C, Richardson D. Fish oil increases peripheral capillary blood cell velocity in humans. Atherosclerosis 1987; 66:237-245.

18. Jenkins RR. Free radical chemistry. Relation to exercise. Sports Med 1988; 5:156-170.

19. AI MDM, van Houwelingen AC, Kester ADM, Hasaart THM, de Jong AEP, Homstra G. Matemal essential fatty acid pattems during normal pregnancy and their relationship to the neonatal essential fatty acid status. Br J Nutr 1995; 74:55-68.

20. Roberts JM, Taylor RN, Musci JT, Rodgers GM, Hubel CA, McLauglin MK. Preeclampsia: an endothelial cell disorder Am J Obstet Gynecol 1989; 161:1200-1204. 


\title{
Effects of fish oil and vitamin E supplementation on copper- catalyzed oxidation of human low density lipoprotein in vitro
}

\author{
Gerard S. Oostenbrug, Ronald P. Mensink \& Gerard Hornstra
}

Based on: European Joumal of Clinical Nutrition 1994; 48:895-898.

\section{ABSTRACT}

The highly unsaturated fish oils have beneficial health effects, but may increase the susceptibility of low density lipoproteins (LDLs) to oxidative modification. We, therefore, investigated this potentially atherogenic effect and the influence of the natural antioxidant vitamin $\mathrm{E}$ in humans.

Seven men received a daily supplement of $6 \mathrm{~g}$ of fish oil for 3 weeks; four of them received an additional $300 \mathrm{mg}$ of vitamin $\mathrm{E}$. Four additional men received no supplements, and served as controls. LDL was isolated before, during and after the supplementation period, and oxidized in vitro with $\mathrm{CuCl}_{2}$. Oxidation was measured spectrophotometrically by continuously monitoring the formation of conjugated dienes at $234 \mathrm{~nm}$.

After three weeks, the mean $( \pm S D$ ) amount of dienes formed in vitro (oxidizability) had increased in the fish oil group ( $20 \pm 6 \% ; P=0.006$ vs control group) and fish oil/vitamin $E$ group ( $18 \pm 4 \% ; P=0.008$ vs control group), but not in the control group ( $1 \pm 9 \%$ ).

The lagtime before onset of oxidation (oxidation resistance) had decreased by $22.7 \pm 8.4$ minutes $(29 \pm 7 \%)$ in the fish oil group, but had increased by $5.6 \pm 14.6$ minutes $(6 \pm 18 \%)$ in the fish oilvitamin $E$ group ( $P=0.008$ between groups). These changes, however, did not differ significantly from the decrease in the control group $(7.5 \pm 6.8$ minutes or $11 \pm 9 \%$; $P=0.10$ vs fish oil group; $P=0.12$ vs fish oil/vitamin $E$ group).

The rate of oxidation tended to be lower in the fish oil/vitamin $E$ group, but ditferences between groups did not reach statistical significance.

We conclude that in man, dietary fish oil increases the copper-induced oxidizability of LDL in vitro, and that vitamin $\mathrm{E}$ may prevent this potentially adverse condition by increasing the resistance against oxidative modification. 


\section{INTRODUCTION}

Reduction of plasma low density lipoproteins (LDL) can be achieved by replacing saturated fatty acids in the diet with linoleic acid, the most common $n-6$ polyunsaturated fatty acid (PUFA) ${ }^{1}$. Increasing the proportion of dietary linoleic acid causes an increase in the proportion of linoleic acid in the LDL particle ${ }^{2,3}$. This may present an unfavorable condition, because LDL can be modified by oxidation of its PUFAs ${ }^{4}$, and several studies have suggested that oxidatively modified LDL is more atherogenic than native LDL (for reviews, see refs 5,6 , and 7).

So far, most studies have compared the effects on LDL oxidation in vitro of dietary linoleic acid versus oleic acid, a monounsaturated fatty acid $^{3,8,9,10}$. Indeed it was found that a linoleic acid rich diet increased the susceptibility of LDL to oxidation in vitro. Less is known about the effect of dietary fish oil on LDL oxidation. Fish oils are rich in n-3 PUFAs, which may be beneficial to human health ${ }^{11}$, but which may, on the other hand, be very susceptible to oxidation because of their high degree of unsaturation. The present study therefore tested the effects of a dietary fish oil supplement on the susceptibility of LDL to copper-catalyzed oxidation in vitro.

Apart from its PUFA content, the antioxidant status of the LDL particle determines the susceptibility to oxidation as well ${ }^{12,13,14}$. We therefore also examined whether supplementation with vitamin $\mathrm{E}$, a natural, lipid-soluble antioxidant, could counteract the expected decrease in LDL oxidation resistance caused by an increased intake of dietary fish oil.

\section{METHODS}

\section{Design, subjects and dietary supplements}

Twelve male volunteers participated in this experiment. All were in good health, and none was taking any medication known to affect serum lipids. The protocol and aim of the study were fully explained, and all gave their written informed consent. Approval for the study was obtained from the Medical Ethics Committee of the University of Limburg. 
For three weeks, eight of the men received, three times per day, two capsules, each containing 1 gram of a fish oil concentrate (ETOS, Zaandam, The Netherlands). These capsules provided an additional $1.3 \mathrm{~g}$ of eicosapentaenoic acid (EPA; C20:5 $\mathrm{n}-3$ ) and $0.8 \mathrm{~g}$ of docosahexaenoic acid (DHA; C22:6 n-3) per day (Table 2.1). Four of these men also received a daily supplement of $300 \mathrm{mg}$ ( $300 \mathrm{IU}$ ) of vitamin $\mathrm{E}$ (dl- $\alpha$-tocopherol-acetate: "Ephynal 300" capsules, a generous gift of Hoffmann-La Roche, Basel, Switzerland). Capsules were handed out weekly. The men were asked to keep the capsules refrigerated, and to take out only the number necessary for one day. The remaining four men did not receive any capsules and served as a control group. All participants were urged not to change their habitual diets. On two consecutive days, 1 or 2 men from each of the three experimental groups entered the study on the same day. During the first week one man from the fish oil group withdrew from the study for reasons of illness.

Body weights were recorded weekly, prior to blood sampling. The 11 participants who completed the study were between 19 and 53 years of age (mean: 32 years). Body weights at the start of the study, ranged from 66 to $85 \mathrm{~kg}$ (mean: $78 \mathrm{~kg}$ ) and body mass indexes from 17.6 to $27.3 \mathrm{~kg} / \mathrm{m}^{2}$ (mean: $23.2 \mathrm{~kg} / \mathrm{m}^{2}$ ). Baseline plasma LDL cholesterol levels ranged from 1.64 to $3.54 \mathrm{mmol} / \mathrm{l}$ (mean: $2.38 \mathrm{mmol} / \mathrm{l}$ ). One participant smoked.

\section{Blood sampling and analysis}

After subjects had fasted for 12 hours, blood samples from an antecubital vein were obtained on days $1,8,15$ and 22 in $10 \mathrm{ml}$ vacutainer tubes with a final concentration of $4.7 \mathrm{mmol} / \mathrm{K} \mathrm{K}_{3}$ EDTA when filled with blood ("Venoject", Terumo Europe, Leuven, Belgium). Plasma was obtained after 15 minutes of centrifugation at $2000 \mathrm{~g}$ and $4{ }^{\circ} \mathrm{C}$ within one hour of venipuncture.

Isolation of LDL from fresh plasma was started on the same day by single spin density gradient ultracentrifugation ${ }^{15}$. Sudan Black $B$ to prestain the lipoproteins, however, was not added because it interfered with the LDL oxidation analysis (unpublished observations). All gradient solutions contained $1.0 \mathrm{~g} / /(2.7 \mathrm{mmol} / \mathrm{l})$ of $\mathrm{Na}_{2}$ EDTA.2 $\mathrm{H}_{2} \mathrm{O}$ to prevent initiation of oxidation of the LDL particle during isolation. LDL $(\rho=1.019-1.055 \mathrm{~g} / \mathrm{ml}$ ) was collected by aspiration, and $0.6 \mathrm{ml}$ of each sample was 
immediately dialyzed for 24 hours against a 100 -fold volume of dialysis buffer (10 mmol// KH $\mathrm{KO}_{4}, 0.15 \mathrm{~mol} / \mathrm{NaCl}, \mathrm{pH} 7.4,0.1 \mathrm{mg} / \mathrm{l}$ chloramphenicol) ${ }^{12}$, which was made oxygen-free by purging with nitrogen. Samples collected on the same day were dialyzed together in one buffer solution (60 $\mathrm{ml}$ buffer per sample). Dialysis was performed at $4{ }^{\circ} \mathrm{C}$ in Visking V8 dialysis tubing (Radiometer Holland, Zoetermeer, The Netherlands) under a nitrogen atmosphere in the dark. The buffer was changed once, after 8 hours. Cholesterol concentrations in the LDL fractions were determined colorimetrically, immediately after isolation (Monotest Cholesterol, Boehringer Mannheim, Mannheim, Germany). After dialysis, samples were diluted with oxygenpurged phosphate-buffered saline (PBS: $10 \mathrm{mmol} / / \mathrm{KH}_{2} \mathrm{PO}_{4}, 0.15 \mathrm{~mol} / \mathrm{l} \mathrm{NaCl}, \mathrm{pH}$ 7.4) to a final concentration of $0.26 \mathrm{mmol}$ cholesterol/l, and oxidation was initiated with $\mathrm{CuCl}_{2}$ (final concentration $1.66 \mu \mathrm{mol} / /$ ) in a quartz cuvette. Oxidation of PUFAs was measured spectrophotometrically by monitoring the formation of conjugated fatty acid dienes at $37^{\circ} \mathrm{C}^{4,12}$. The change in absorbance at $234 \mathrm{~nm}$ was recorded over a 5 -hour period, and the amount of dienes was calculated using a molar extinction coefficient for conjugated dienes of $\varepsilon_{234}=29,500 \mathrm{l} / \mathrm{mol} / \mathrm{cm}$ (for more detailed information, see ref. 12).

Oxidation of $L D L$ in vitro was described by the maximum amount of conjugated dienes formed, by the lagtime between the addition of copper to initiate oxidation and the onset of rapid oxidation, and by the maximum rate of formation of conjugated dienes during this rapid oxidation (propagation phase) ${ }^{12}$. Because samples from each experimental group were present in each LDL isolation, dialysis, and oxidation run, differences between the groups due to analytical variation were minimized.

Aliquots of plasma and LDL at baseline and at the end of the study were stored at $-80^{\circ} \mathrm{C}$ for further analyses. Vitamin $\mathrm{E}$ levels in LDL were determined by high performance liquid chromatography $(\mathrm{HPLC})^{16}$. LDL $(200 \mu \mathrm{l})$ was mixed with retinol acetate $(50 \mu \mathrm{l}, 15 \mathrm{mg} / \mathrm{l})$ as intemal standard, ethanol $(200 \mu \mathrm{l})$ and methanol $(200 \mu \mathrm{l})$. Then $\mathrm{n}$-hexane $(0.5 \mathrm{ml})$ was added, the samples were vortexed for 1 minute, and centrifuged at $4000 \mathrm{rpm}(2700 \mathrm{~g})$ for 5 minutes. The organic top phase was removed and the extraction repeated. The pooled hexane phase was evaporated to dryness under nitrogen. The residue was redissolved in $500 \mu \mathrm{l}$ methanol, and an aliquot of 50

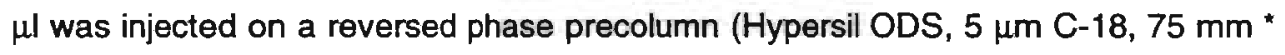
$4.6 \mathrm{~mm}$ I.D.; Chrompack, Middelburg, The Netherlands) followed by a reversed phase column (Hypersil ODS, $5 \mu \mathrm{m} \mathrm{C-18,} 150 \mathrm{~mm}$ * $4.6 \mathrm{~mm}$ I.D.; Chrompack), and eluted 
with $99 \%$ methanol at a flow rate of $1.0 \mathrm{~m} / \mathrm{min}$ using a Spectroflow 400 pump (Separations, H.I. Ambacht, The Netherlands). Detection wavelength (Spectroflow 783, Separations) was set at $340 \mathrm{~nm}$ (retinol acetate) during the first 5 minutes, and at 280 $\mathrm{nm}$ (vitamin E) during the 5 minutes thereafter. Peak areas were calculated by a Spectra-Physics 4400 integrator and calibrated against standard $\alpha$-tocopherol solutions $(2.5,5$ and $10 \mathrm{mg} / \mathrm{l}$ in ethanol). Vitamin E levels were lipid-standardized using (LDL) cholesterol values.

Due to limited amounts of LDL, no fatty acid composition of LDL could be measured. It is conceivable, however, that the fatty acid composition of LDLs and plasma phospholipids changed similarly during the study. The fatty acid composition of plasma phospholipids was determined by gas chromatography. Briefly, total lipid was extracted from $100 \mu$ l plasma samples with $3 \mathrm{ml}$ methanol:chloroform $(1: 2, \mathrm{vol}: \mathrm{vol})$ via a modified Folch method ${ }^{17,18}$. Dinonadecanoyl phosphatidylcholine (31 $\mu \mathrm{g}$ per $100 \mu$ l plasma; Avanti, Alabaster, USA) was used as internal standard. Phospholipids were separated from the total lipid extract with a Bond Elut $\mathrm{NH}_{2}$-aminopropyl column (Rochrom, Rotterdam, The Netherlands) ${ }^{19}$, and then saponified and methylated with $14 \%$ boron trifluoride in methanol (Fluka Chemie AG, Buchs, Switzerland) at $100{ }^{\circ} \mathrm{C}$ for 1 hour ${ }^{20}$. The fatty acid methylesters were extracted with pentane, evaporated to dryness, and redissolved in $50 \mu \mathrm{l}$ isooctane. Three microliters (split ratio of 1:40) were then injected on a HP 5890 II gas chromatograph fitted with a $50 \mathrm{~m}, 0.25 \mathrm{~mm}$ I.D., $0.12 \mu \mathrm{m}$ film thickness, CP-Sil $5 \mathrm{CB}$ non-polar capillary column (Chrompack), using $\mathrm{N}_{2}$ as carrier gas. A standard mixture of fatty acid methylesters was used to optimize the temperature programming, and to identify the fatty acid methylesters by relative retention times. Fatty acids were quantified based on the peak areas relative to the intemal standard. To measure its fatty acid composition, the fish oil concentrate was directly methylated, with trinonadecanoin as internal standard, and the methylesters were analyzed as described above.

All chemicals were purchased from Merck (Darmstadt, Germany) or Sigma Chemical Company (Saint Louis, USA), unless otherwise indicated. Methanol used for HPLC was of HPLC grade. 


\section{Statistical analysis}

For LDL oxidation parameters, responses to the supplements were calculated per subject as the changes from day 1 to days 8,15 and 22, and for all other parameters from day 1 to day 22 . Thus, each subject served as his own control and interindividual variation was excluded. Differences at baseline and differences in responses between the experimental groups were examined by oneway analysis of variance using the General Linear Models procedure of the Statistical Analysis System ${ }^{21}$. Pearson correlation coefficients were calculated between various parameters at baseline. Values are reported as mean \pm SD.

\section{RESULTS}

No significant differences between the experimental groups were present at the start of the study for any of the parameters studied (Table 2.1 and 2.2). Over the 3 weeks of the study, slight increases in body weight $(0.6 \pm 0.9 \mathrm{~kg})$ and LDL cholesterol $(0.16 \pm 0.24 \mathrm{mmo} / \mathrm{l})$ were noticed. Changes, however, were not significantly different between the groups.

Compliance to the supplements was confirmed by a significant increase in the proportion of EPA ( $P \leq 0.0001$ vs control group) and DHA ( $P=0.0004$ and $P=0.0002$ for respectively the fish oil and the fish oil/vitamin $E$ group vs the control group) in the plasma phospholipids of the fish oil supplemented volunteers (Table 2.1), and by an increase of vitamin E in LDL of the fish oil/vitamin E supplemented group ( $P \leq 0.0001$ vs both other groups; Table 2.2). The increase in the proportion of $n-3$ fatty acids was accompanied by a decrease in the proportion of $n-6$ fatty acids. Although the total amount of PUFAs was therefore similar in all groups, the total number of double bonds contained in the PUFAs (PUFA unsaturation index) was higher in the two groups receiving the fish oil supplement $(P=0.003$ and $P=0.002$ for respectively the fish oil and the fish oil/vitamin $E$ group vs the control group; Table 2.1). 
Table 2.1. Fatty acid composition of the fish oil concentrate and the effects on the fatty acid composition of plasma phospholipids after three weeks of fish oil supplementation.

Plasma phospholipid fatty acids in experimental groups

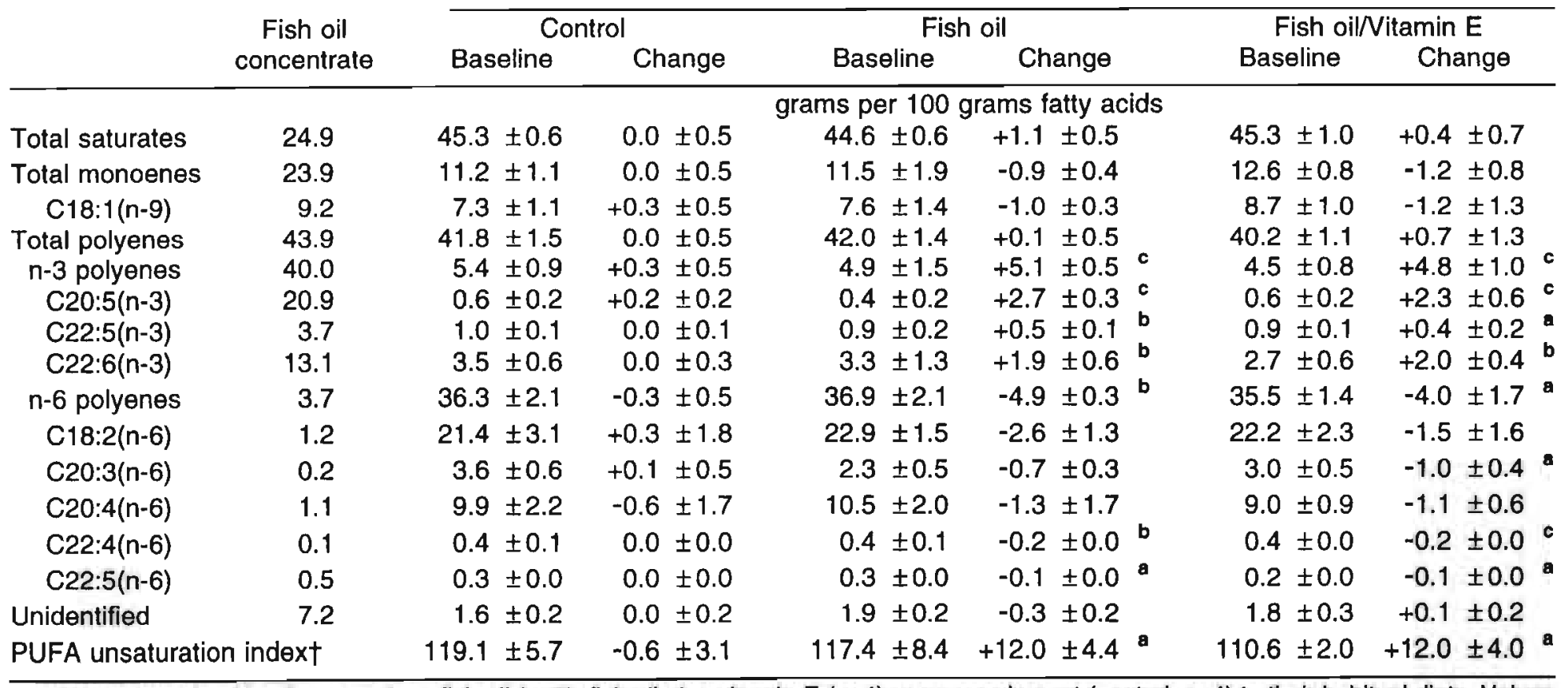

Eleven men received for three weeks a fish oil $(n=3)$, fish oil plus vitamin $E$ ( $n=4$ ) or no supplement (control, $n=4)$ to their habitual diets. Values for phospholipid fatty acid composition are means \pm SD. Asterisks indicate significant differences in change from control group (oneway analysis of variance): $a: P<0.01 ; b: P<0.001 ; c: P \leq 0.0001$. No significant differences were noticed at baseline between the three groups, nor in change between the fish oil and fish oil/vitamin $E$ group.

† Calculated as the sum of the percentage of each PUFA (moles/moles total fatty acids * $100 \%$ ) multiplied with its number of double bonds. 
Table 2.2. Effects of fish oil supplementation, with or without vitamin $E$, on cholesterol levers, cholesterol standardized vitamin $E$ levels and copper-catalyzed oxidation of low density lipoprotein (LDL) in vitro.

Experimental group

\begin{tabular}{|c|c|c|c|c|c|c|}
\hline & Control & & sh oil & Fish oil & vita & $\min E$ \\
\hline \multicolumn{7}{|c|}{$\overline{\mathrm{LDL}}$ cholesterol (mmol/) } \\
\hline baseline & $2.48 \pm 0.79$ & 2.33 & \pm 0.59 & 2.33 & \pm & 0.73 \\
\hline end & $2.55 \pm 1.00$ & 2.43 & \pm 0.60 & 2.63 & \pm & 0.56 \\
\hline change & $+0.07 \pm 0.26$ & +0.10 & \pm 0.13 & +0.31 & \pm & 0.27 \\
\hline \multicolumn{7}{|c|}{ LDL vitamin $E$ (mg vitamin E/mmol LDL cholesterol) } \\
\hline baseline & $1.56 \pm 0.62$ & 1.54 & \pm 0.42 & 1.18 & \pm & 0.19 \\
\hline end & $1.70 \pm 0.51$ & 1.78 & \pm 0.30 & 2.90 & \pm & 0.28 \\
\hline change & $+0.14 \pm 0.19^{c}$ & +0.24 & $\pm 0.12^{c}$ & +1.73 & \pm & $0.19^{\circ}$ \\
\hline
\end{tabular}

LL oxidation:

Conjugated dienes ( $\mu \mathrm{mol}$ dienes/mmol LDL cholesterol)

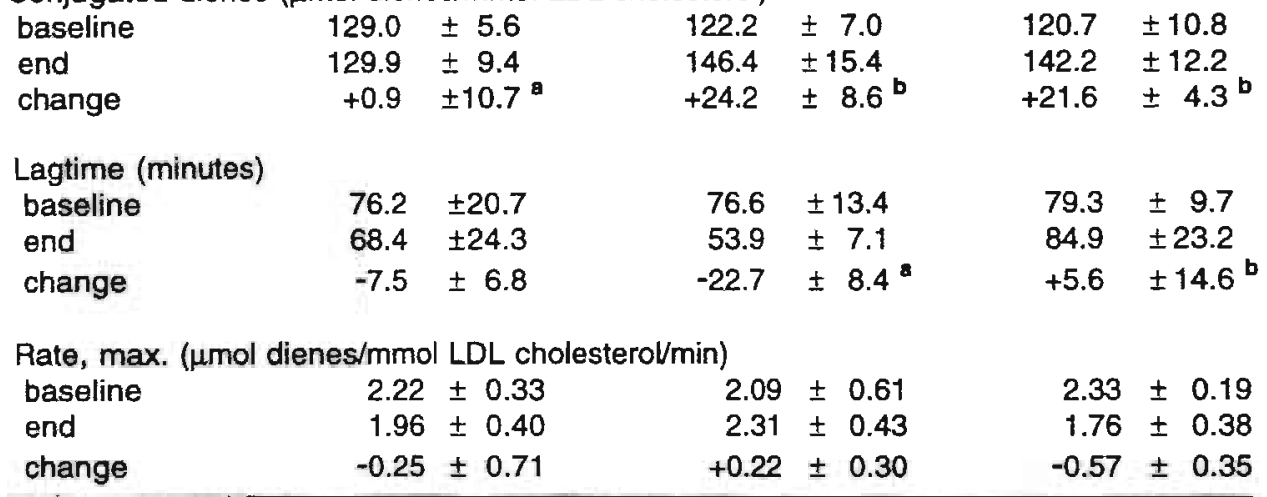

Values are means \pm SD. Male subjects received no supplement (control group, $n=4$ ), or 6 g/day of a fish oil supplement, without (fish oil group, $n=3$ ) or with (fish oilvitamin $E$ group, $n=4$ ) 300 Ul/day vitamin E. Baseline values and changes over the 3 weeks of the study were compared by oneway analysis of variance: superscripts $b$ and $c$ indicate significant differences from a-superscript: $a$ vs $b$ : $P<0.01$; a vs $c: P \leq 0.0001$. ", $n=3$ for $L D L$ vitamin $E$ analysis (one sample lost during analysis).

Within one week, the amount of dienes formed during oxidation of LDL in vitro, was higher in both groups on fish oil supplementation (Figure 2.1, top panel). After three weeks, the amount of dienes in the fish oil group had increased by $20 \%$ (24.2 $\pm 8.6 \mu \mathrm{mol}$ dienes $/ \mathrm{mmol} \mathrm{LDL}$ cholesterol; $\mathrm{p}=0.006 \mathrm{vs}$ control group) and in the fish oilvitamin $E$ group by $18 \%(21.6 \pm 4.3 \mu \mathrm{mol}$ dienes $/ \mathrm{mmol}$ LDL cholesterol; $\mathrm{P}=0.008$ vs control group), while the change in the control group was less than $1 \%$ (0.9 $\pm 10.7 \mu \mathrm{mol} / \mathrm{mmol}$ LDL cholesterol; Table 2.2). 

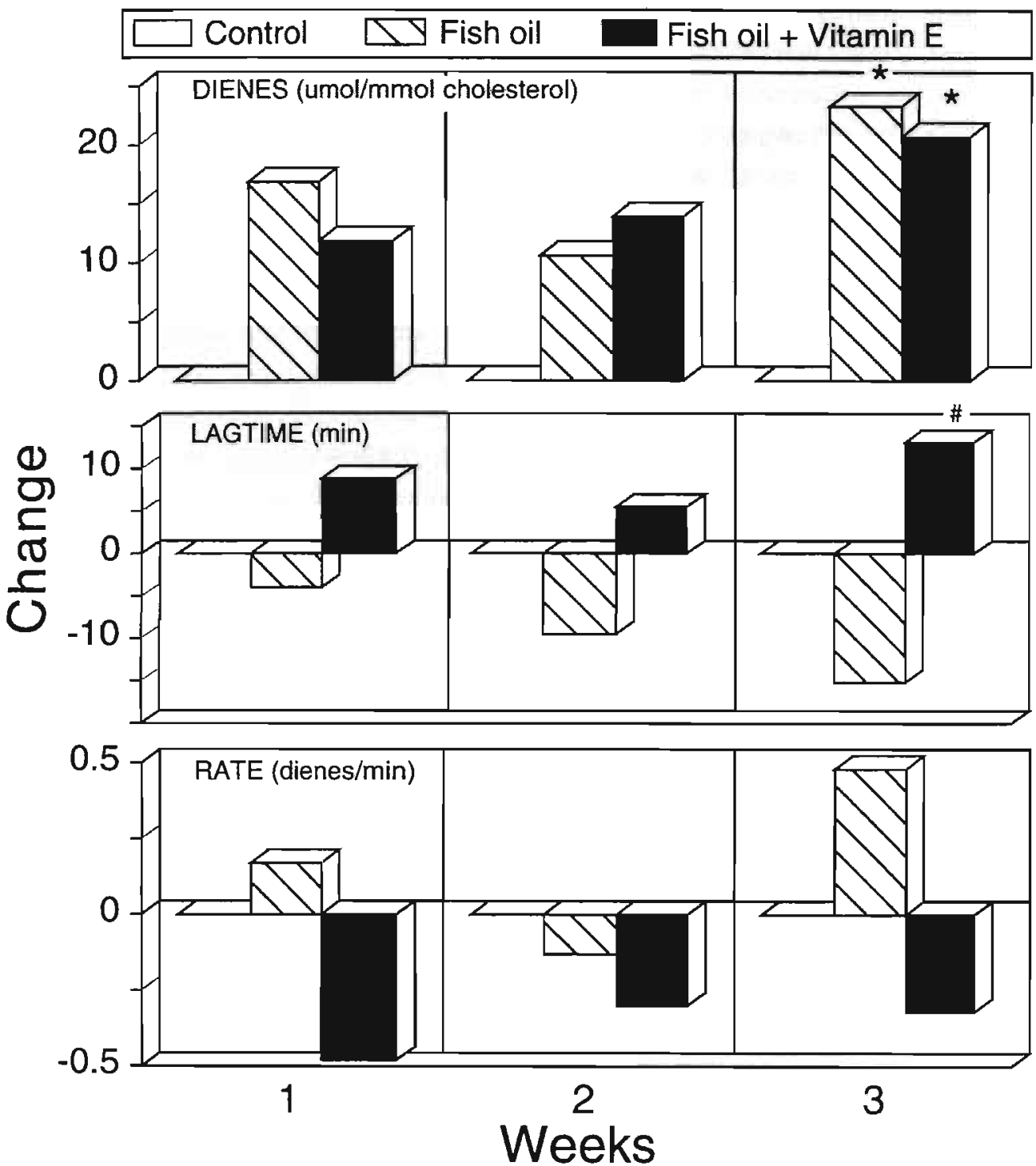

Figure 2.1. Effect of fish oil and vitamin $E$ supplementation on maximum amount of conjugated dienes (top panel), lagtime before onset of oxidation (center panel), and maximum rate of diene formation (bottom panel) during oxidation of human low density lipoprotein in vitro. Results are shown as mean changes relative to the start of the study. Changes are corrected for unknown drifts with time as measured by the changes in the control group, which are set to zero. ", \# Significant difference $(P<0.01)$ : * vs control group, \# vs fish oil group. 
Compared to the control group, the lagtime decreased in the fish oil group and increased in the fish oil/vitamin E group (Figure 2.1, center panel). After three weeks, the lagtime in the control group had decreased by $7.5 \pm 6.8$ minutes $(11 \pm 9 \%)$. The lagtime in the fish oil group, however, had decreased by $22.7 \pm 8.4$ minutes $(29 \pm 7 \%$; $P=0.10$ vs control group), whereas the fish oil/vitamin $E$ group showed an increase of $5.6 \pm 14.6$ minutes $(6 \pm 18 \% ; P=0.008$ vs fish oil group; $P=0.12$ vs control group; Table 2.2).

The maximum rate of diene formation during oxidation decreased after 3 weeks by $0.57 \pm 0.35 \mu \mathrm{mol}$ dienes $/ \mathrm{mmol} \mathrm{LDL}$ cholesterol/min in the fish oil/vitamin $E$ group ( $P=0.40$ vs control group; $P=0.07$ vs fish oil group). Mean changes were $-0.25 \pm 0.71$ in the control group and $+0.22 \pm 0.30 \mu \mathrm{mol}$ dienes $/ \mathrm{mmol} \mathrm{LDL}$ cholesterol $/ \mathrm{min}$ in the fish oil group ( $P=0.25$ between groups; Table 2.2). Differences between groups were not consistent throughout the study, but decreases were always most pronounced in the fish oilvitamin E group (Figure 2.1, bottom panel).

At baseline, no significant correlations were found between the cholesterol standardized vitamin E concentration in LDL $(n=10)$ and lagtime $(r=0.304 ; P=0.39)$, amount of dienes $(r=0.490 ; P=0.15)$ or rate of diene formation $(r=0.294 ; P=0.41)$.

\section{DISCUSSION}

The present study examined the effects of dietary fish oil and vitamin E on coppercatalyzed in vitro oxidation of human LDL. Three parameters were measured to determine the extent of oxidation.

\section{Maximum amount of conjugated dienes (oxidizability)}

Increasing the dietary fish oil intake for three weeks caused a marked increase in the amount of conjugated dienes formed during copper-catalyzed oxidation of LDL in vitro. This increased oxidizability of LDL was also found when the proportion of dietary n-6 PUFAs (i.c. linoleic acid) was increased at the expense of oleic acid, both in rabbits ${ }^{8}$ and in $\operatorname{man}^{3,9,10}$. Only a few studies looked at the effects of dietary $n-3$ 
PUFAs on LDL oxidation in vitro. Nenseter et al. ${ }^{2}$ analyzed LDL samples for lipid peroxides after oxidation with copper for 1,6 , and 24 hours. The levels of lipid peroxides in samples from two volunteers receiving a fish oil supplement did not differ from samples from two volunteers receiving a com oil supplement, although the unsaturation index of LDL was higher in the fish oil group. Due to the small number of samples and the absence of baseline values, however, no final conclusion can be drawn from this part of the study. Harats et al. ${ }^{22}$ found increased levels of thiobarbituric acid reactive substances (TBArs) in native and in vitro oxidized LDL of fish oil supplemented volunteers as compared with not-supplemented volunteers. Oxidation of LDL was performed by a fixed period of incubation with either copper or smooth muscle cells. Additional dietary supplementation with vitamin $E$ (fish oil + vitamin $E$ group) attenuated the increase in TBArs caused by the fish oil. In the present study, vitamin $\mathrm{E}$ did not affect the maximum amount of dienes formed in vitro, but only increased the time needed to reach this maximum. Levels of TBArs (or other oxidation products) after a fixed period of oxidation, however, may not necessarily represent maximum levels. Indeed, two studies showed that supplementation with vitamin $E^{23}$, or probucol, another chain-breaking antioxidant ${ }^{24}$, decreased levels of both TBArs and conjugated dienes in LDL measured after 3 hours of oxidation in vitro in PBS, but that maximum levels (after 5 or 8 hours of oxidation) were similar to maximum levels in the placebo supplemented group. The lower pre-maximum values in the vitamin $E$ and probucol supplemented groups can (at least partly) be explained by the significantly lower rate of oxidation in the vitamin $E$ group (ref. 23, lagtime not determined), and by a significantly longer lagtime in the probucol group (ref. 24, rate of oxidation not determined). In a recent study of Lussier-Cacan et $a .^{25}$, the effect of dietary fish oil and the antioxidant probucol on copper-catalyzed oxidation of LDL in vitro was studied. Six hypertriglyceridemic patients (3 with type III, and 3 with type IV) received a low-saturated-fat, low-cholesterol diet with no supplement, a fish oil supplement, and a fish oil plus probucol supplement in three consecutive periods. TBArs were measured each hour during the 5 hours of oxidation, and in the fish oil period, levels of TBArs in LDL were systematically higher as compared with the not-supplemented period. Additional supplementation with probucol drastically decreased the level of TBArs. Although a clear maximum was not reached after 5 hours of oxidation, their data indicated that the propagation phase was near the end, thus a further large increase of TBArs in the fish oillprobucol period seems 


\section{REFERENCES}

1. Mensink RP, Katan MB. Effect of dietary fatty acids on serum lipids and lipoproteins. A metaanalysis of 27 trials. Arterioscler Thromb 1992; 12:911-919.

2. Nenseter MS, Rustan AC, Lund-Katz S, Søyland E, Mælandsmo G, Phillips MC, Drevon CA. Effect of dietary supplementation with n-3 polyunsaturated fatty acids on physical properties and metabolism of low density lipoprotein in humans. Arterioscler Thromb 1992; 12:369-379.

3. Abbey M, Belling GB, Noakes M, Hirata F, Nestel PJ. Oxidation of low-density lipoproteins: Intraindividual variability and the effect of dietary linoleate supplementation. Am J Clin Nutr 1993; 57:391-398.

4. Halliwell $B$, Gutteridge JMC. The importance of free radicals and catalytic metal ions in human diseases. Mol Aspects Med 1985; 8:89-193.

5. Steinberg D, Parthasarathy S, Carew TE, Khoo JC, Witztum JL. Beyond cholesterol. Modifications of low-density lipoprotein that increase its atherogenicity. N Engl J Med 1989; 320:915-924.

6. Steinbrecher UP, Zhang $H$, Lougheed $M$. Role of oxidative modified LDL in atherosclerosis. Free Rad Biol Med 1990; 9:155-168.

7. Parthasarathy S, Rankin SM. Role of oxidized low density lipoprotein in atherosclerosis. Prog Lipid Res 1992; 31:127-143.

8. Parthasarathy S, Khoo JC, Miller E, Barnett J, Witztum JL, Steinberg D. Low density lipoprotein rich in oleic acid is protected against oxidative modification: Implications for dietary prevention of atherosclerosis. Proc Natl Acad Sci USA 1990; 87:3894-3898.

9. Reaven P, Parthasarathy S, Grasse BJ, Miller E, Almazan F, Mattson FH, Khoo JC, Steinberg D, Witztum JL. Feasibility of using an oleate-rich diet to reduce the susceptibility of low-density lipoprotein to oxidative modification in humans. Am J Clin Nutr 1991; 54:701-706.

10. Reaven P, Parthasarathy S, Grasse BJ, Miller E, Steinberg D, Witztum JL. Effects of oleate-rich and linoleate-rich diets on the susceptibility of low density lipoprotein to oxidative modification in mildly hypercholesterolemic subjects. J Clin Invest 1993; 91:668-676.

11. Leaf A, Weber PC. Medical progress. Cardiovascular effects of $n-3$ fatty acids. N Engl J Med 1988; 318:549-557.

12. Esterbauer H, Striegl G, Puhl H, Rotheneder M. Continuous monitoring of in vitro oxidation of human low density lipoprotein. Free Rad Res Commun 1989; 6:67-75.

13. Esterbauer $H$, Dieber-Rotheneder $M$, Striegl $G$, Waeg $G$. Role of vitamin $E$ in preventing the oxidation of low-density lipoprotein. Am J Clin Nutr 1991; 53:314S-321S.

14. Dieber-Rotheneder $M$, Puhl $H$, Waeg G, Striegl G, Esterbauer $H$. Effect of oral supplementation with $D$ - $\alpha$-tocopherol on the vitamin $E$ content of human low density lipoproteins and resistance to oxidation. J Lipid Res 1991; 32:1325-1332.

15. Terpstra AHM, Woodward $\mathrm{CJH}$, Sanchez-Muniz FJ. Improved techniques for the separation of serum lipoproteins by density gradient ultracentrifugation: Visualization by prestaining and rapid separation of serum lipoproteins from small volumes of serum. Anal Biochem 1981; 111:149-157.

16. Cuesta Sanz D, Castro Santa-Cruz M. Simultaneous measurement of retinol and $\alpha$-tocopherol in human serum by high-performance liquid chromatography with ultraviolet detection. J Chromatogr 1986; 380:140-144.

17. Folch J, Lees $\mathrm{M}$, Sloane Stanley GH. A simple method for the isolation and purification of total lipides from animal tissues. J Biol Chem 1957; 226:497-509. 
18. Hoving EB, Jansen G, Volmer M, van Doormaal JJ, Muskiet FAJ. Profiling of plasma cholesterol ester and triglyceride fatty acids as their methylesters by capillary gas chromatography, preceded by a rapid aminopropylsilica column chromatographic separation of lipid classes. $J$ Chromatogr 1988; 434:395-409.

19. Kaluzny MA, Duncan LA, Merritt MV, Epps DE. Rapid separation of lipid classes in high yield and purity using bonded phase columns. J Lipid Res 1985; 26:135-140.

20. Morrison WR, Smith LM. Preparation of fatty acid methyl esters and dimethylacetals from lipids with boron fluoride-methanol. J Lipid Res 1964; 5:600-608.

21. SAS Institute Inc., SAS/STAT User's Guide, Version 6, Fourth Edition, Vol. 2. Cary NC: SAS Institute Inc., 1989.

22. Harats D, Dabach Y, Hollander G, Ben-Naim M, Schwartz R, Berry EM, Stein O, Stein Y. Fish oil ingestion in smokers and nonsmokers enhances peroxidation of plasma lipoproteins. Atherosclerosis 1991; 90:127-139.

23. Jialal I, Grundy SM. Effect of dietary supplementation with alpha-tocopherol on the oxidative modification of low density lipoprotein. J Lipid Res 1992; 33:899-906.

24. Cristol LS, Jialal I, Grundy SM. Effect of low-dose probucol therapy on LDL oxidation and the plasma lipoprotein profile in male volunteers. Atherosclerosis 1992; 97:11-20.

25. Lussier-Cacan S, Dubreuil-Quidoz S, Roederer G, Leboeuf N, Boulet L, de Langavant GC, Davignon $J$, Naruszewicz $M$. Influence of probucol on enhanced LDL oxidation after fish oil treatment of hypertriglyceridemic patients. Arterioscler Thromb 1993; 13:1790-1797.

26. Cominacini L, Garbin U, Cenci B, Davoli A, Pasini C, Ratti E, Gaviraghi G, Lo Cascio V, Pastorino AM. Predisposition to LDL oxidation during copper-catalyzed oxidative modification and its relation to $\alpha$-tocopherol content in humans. Clin Chim Acta 1991; 204:57-68.

27. Jessup W, Rankin SM, De Whalley CV, Hoult JRS, Scott J, Leake DS. $\alpha$-Tocopherol consumption during low-density-lipoprotein oxidation. Biochem J 1990; 265:399-405.

28. Princen HMG, van Poppel G, Vogelezang C, Buytenhek R, Kok FJ. Supplementation with vitamin $E$ but not $\beta$-carotene in vivo protects low density lipoprotein from lipid peroxidation in vitro. Effect of cigarette smoking. Arterioscler Thromb 1992; 12:554-562.

29. Hepbum FN, Exler J, Weihrauch JL. Provisional tables on the content of omega-3 fatty acids and other fat components of selected foods. J Am Diet Assoc 1986; 86:788-793.

30. Millet $P$, Guilland JC, Fuchs F, Klepping J. Nutrient intake and vitamin status of healthy French vegetarians and nonvegetarians. Am J Clin Nutr 1989; 50:718-727.

31. Bolton-Smith C, Woodward M, Tunstall-Pedoe $H$. The Scottish Heart Health Study. Dietary intake by food frequency questionnaire and odds ratios for coronary heart disease risk. II. The antioxidant vitamins and fibre. Eur $J$ Clin Nutr 1992; 46:85-93.

32. Berlin E, Bhathena SJ, Judd J, Nair PP, Peters RC, Bhagavan HN, Ballard-Barbash R, Taylor PR. Effects of omega-3 fatty acid and vitamin E supplementation on erythrocyte membrane fluidity, tocopherols, insulin binding, and lipid composition in adult men. J Nutr Biochem 1992; 3:392-400.

33. Ylä-Herttuala S, Palinski W, Rosenfeld ME, Parthasarathy S, Carew TE, Butler S, Witztum JL, Steinberg $D$. Evidence for the presence of oxidatively modified low density lipoprotein in atheroscierotic lesions of rabbit and man. J Clin Invest 1989; 84:1086-1095.

34. Ylä-Herttuala S, Palinski W, Rosenfeld ME, Steinberg D, Witztum JL. Lipoproteins in normal and atherosclerotic aorta. Eur Heart J 1990; 11(suppl E):88-99.

35. Ylă-Herttuala S, Palinski W, Butler SW, Picard S, Steinberg D, Witztum JL. Rabbit and human atherosclerotic lesions contain IgG that recognizes epitopes of oxidized LDL. Arterioscler Thromb $1994 ; 14: 32-40$.

36. Regnström J, Nilsson J, Tornvall P, Landou C, Hamsten A. Susceptibility to low-density lipoprotein oxidation and coronary atherosclerosis in man. Lancet 1992; 339:1183-1186. 



\section{Physical performance, red blood cell characteristics, plasma lipid- soluble antioxidant concentrations and in vitro oxidation of low density lipoproteins during exercise. The effects of fish oil and vitamin E supplementation}

Gerard S. Oostenbrug, Ronald P. Mensink, Max R. Hardeman, Tjeerd de Vries, Fred Brouns \& Gerard Hornstra

Based on: "Exercise performance, erythrocyte characteristics, and lipid peroxidation: effects of fish oil and vitamin E" (submitted).

\section{ABSTRACT}

Previous studies have indicated that fish oil supplementation increases red blood cell (RBC) deformability, which may improve exercise performance. Exercise alone, and the combination with an increase in fatty acid unsaturation, however, may enhance lipid peroxidation, and limit the potentially beneficial effects of fish oil supplementation. Therefore, we investigated the effects of exercise and fish oil supplementation, without (FO) or with vitamin $\mathrm{E}$ (FE), on RBC characteristics, plasma lipid-soluble antioxidant concentrations, and in vitro oxidation of low density lipoproteins (LDL), and the effects of FO and FE on exercise performance, in trained cyclists. RBC deformability appeared to decrease during endurance exercise. After correction for hemoconcentration, plasma total tocopherol and carotenoid concentrations decreased by $2.9 \%(P=0.012)$ and $4.5 \%(P=0.0008)$, respectively. Endurance exercise did not affect the lagtime and rate of in vitro oxidation of $L D L$, but the maximum amount of conjugated dienes formed decreased by $1.2 \%(P=0.042)$. FO and FE did not affect RBC characteristics or exercise performance. Both supplements decreased the rate of LDL oxidation, and FE delayed oxidation. The amount of dienes, however, was not affected. The supplements also did not change effects of exercise. We conclude that the changes observed during endurance exercise may indicate increased oxidative stress. Fish oil supplementation did not improve endurance performance, but also did not cause or augment adverse changes in antioxidant concentrations or LDL oxidation during exercise. 


\section{INTRODUCTION}

Several studies have indicated that dietary fish oils increase the deformability of red blood cells $(\mathrm{RBC})$ as a result of the incorporation of the highly unsaturated fish oil fatty acids in the membrane ${ }^{1,2}$. This may facilitate the transport of RBC through the microcirculation ${ }^{3}$, leading to improved oxygen supply, reduced lactic acid production, and improved exercise performance. In addition, exercise increases intravascular hemolysis ${ }^{4}$, which may be counteracted by fish oil supplementation ${ }^{5}$.

However, incorporation of the highly unsaturated fatty acids in membranes increases the membranes' susceptibility to lipid peroxidation ${ }^{6}$. Previously, we have reported that the susceptibility to oxidation of low density lipoproteins (LDL) was increased by fish oil supplementation, and that this potentially unbeneficial effect could be counteracted by vitamin $E^{7}$. Lipid peroxidation may be especially important during exercise, since exercise increases the formation of reactive oxygen species capable of oxidizing unsaturated fatty acids leading to altered membrane characteristics (for reviews, see Refs 8 and 9). Indeed, exercise ${ }^{10.11,12}$, and treatment of RBC with malondialdehyde (MDA) ${ }^{13}$, a product of lipid peroxidation, have been shown to decrease RBC deformability. Possible benefits of fish oil supplementation for athletes may therefore be counteracted by increased susceptibility to oxidation of the RBC.

Therefore, we investigated the effects of exercise and fish oil supplementation, with or without the antioxidant vitamin E, on RBC characteristics and lipid peroxidation, and the effects of the supplements on exercise performance.

\section{SUBJECTS AND METHODS}

\section{Study design}

Twenty-four well-trained male athletes (aged 19 to 42 years), who were experienced in cycling, entered the study between January 11 and 26, 1995. They trained at least 2 times per week, and their 'maximal workload capacity' ( $W_{\max }$ ) on a cycle ergometer (see below), exceeded 4 watt $/ \mathrm{kg}$ body weight. All participants were healthy as determined by a medical questionnaire. Written informed consent was 
received from each subject, and prior approval for the study was obtained from the Medical Ethics Committee of the University of Limburg.

Participants were asked to abstain from intense physical activity for 36 hours prior to the measurements, and were instructed not to use any antioxidant supplements during and two weeks preceding the study. At the start of the study, $W_{\max }$ of each participant was determined. Four to seven days later, the subjects performed an endurance exercise test on a cycle ergometer, during which changes in hematological variables, RBC characteristics, plasma viscosity, plasma antioxidant status, and LDL oxidation were measured. For the next 3 weeks, 8 subjects (PLA group) received a placebo supplement, 8 subjects (FO group) a fish oil supplement (6 $\mathrm{g}$ of Fish-EPA daily), and 8 subjects (FE group) the fish oil supplement together with vitamin $E$ (Ephynal 300, $300 \mathrm{IU}$ dl- $\alpha$-tocopherol acetate daily). The three groups were stratified for $W_{\max }$, and, as far as possible, one subject from each group was tested on the same day. At the end of the study, exercise testing procedures were repeated.

Placebo capsules, containing micro-crystalline cellulose, were supplied by the pharmacy of the Academic Hospital Maastricht (Maastricht, The Netherlands). Fish-EPA capsules were a generous gift from Orthica (Weesp, The Netherlands), and Ephynal 300 capsules from Hoffmann-La Roche (Basel, Switzerland). The fish oil capsules contained (w/w) 17.6\% EPA and $12.5 \%$ DHA, and only $0.12 \%$ vitamin $E$ ( $0.01 \% \delta$-tocopherol, $0.03 \% \beta+\gamma$-tocopherol, $0.08 \% \alpha$-tocopherol), as analyzed by gas chromatography and HPLC respectively.

\section{Exercise performance protocols}

Maximal workload capacity test

$W_{\max }$ was determined during an incremental cycle ergometer test with 3 min intervals ${ }^{14}$. The workload corresponding to a plasma lactate concentration of 4 $\mathrm{mmol} / \mathrm{l}$ (anaerobic threshold) was calculated assuming a linear change between the two workloads corresponding to lactate concentrations just below and above this value. Oxygen uptake was measured continuously using a computerized automatic system (Oxycon Delta, Mijnhard, The Netherlands). All $W_{\max }$ tests were performed between 12.00 and 18.00 hour. $W_{\max }$ of each subject, as measured the week before supplementation started, was used for both the endurance exercise tests, before and 
after 3 weeks of supplementation. The second $W_{\max }$ test, performed after 15 to 17 days of supplementation, was used to detect changes in $\mathrm{W}_{\max }$ and plasma lactate concentrations due to the supplements.

\section{Endurance exercise test}

Before and after the 3-week period of supplementation, subjects were asked to perform a prefixed absolute workload on a linearly functioning cycle ergometer (Lode Excalibur Sport, Lode, Groningen, The Netherlands), in as little time as possible. The workload was based on $70 \%$ of the $W_{\max }$ of each subject, and the workload output depended directly on the pedalling frequency. During the test, subjects were only informed about the cumulative achieved amount of work, as displayed by a 0 to $100 \%$ indicator of the total workload. Time as well as pedalling frequency were blinded to the subjects. Subjects were allowed to drink tap water ad libitum. The time needed to complete the test was used as a measure of physical performance. This new validated endurance exercise test, resembling a time trial of approximately 1 hour, has been described in detail by Jeukendrup et al. ${ }^{15}$. All endurance exercise tests were carried out between 8.30 and 11.00 hour.

\section{Blood sampling}

During the $W_{\max }$ tests, an intravascular teflon catheter (Baxter, Utrecht, The Netherlands) was positioned in a fore-arm vein. At the end of each workload step, blood was collected in $\mathrm{Na}_{2}$ EDTA.2 $\mathrm{H}_{2} \mathrm{O}$ (f.c. $2 \mathrm{~g} / \mathrm{l}$ ), and kept on ice. After the test, samples were centrifuged for 10 minutes at $1600 \mathrm{~g}$ and $4{ }^{\circ} \mathrm{C}$, and plasma was stored at $-40{ }^{\circ} \mathrm{C}$ for lactate analysis later.

Before and immediately after each endurance exercise test, fasting free-flowing antecubital venous blood was collected in two decapped $\mathrm{K}_{3}$ EDTA-containing Monojecttubes (f.c. $1.5 \mathrm{~g} /$; Sherwood Medical, Ballymoney, Northem Ireland), while the subjects were in supine position. Blood was also collected in heparin (f.c. 143 U.S.P. units/5 $\mathrm{ml}$; Monoject-tubes, Sherwood Medical) for measurement of osmotic fragility of RBC. Tubes were immediately closed after collection, and blood and anticoagulant were carefully mixed. One tube with EDTA-anticoagulated blood was sent to Amsterdam (ambient temperature), and analyzed the next day for RBC deformability and plasma 
viscosity. Extensive pilot experiments demonstrated that such transport did not significantly affect RBC deformability (results not reported). Hematological variables were analyzed in the other EDTA-tube, which was then centrifuged for 10 minutes at $1300 \mathrm{~g}$ and $4{ }^{\circ} \mathrm{C}$. Part of the fresh EDTA-plasma was used for LDL oxidation, and an aliquot was stored at $-80^{\circ} \mathrm{C}$ for antioxidant analysis. RBC were immediately washed for phospholipid fatty acid analysis.

\section{Blood analyses}

\section{Plasma lactate}

EDTA-plasma, collected during $\mathrm{W}_{\max }$ tests, was analyzed for lactate (enzymatic LDH method; LDH, No. 106984, Boehringer Mannheim, Mannheim) on a COBAS-BIO centrifugal analyzer (F. Hoffmann-La Roche, Basel, Switzerland).

Hematological variables, $R B C$ characteristics, and plasma viscosity

Hematological variables were analyzed on a Coulter MD 18 (Counter, Miami, FL, USA), and changes in blood and plasma volume during endurance exercise were calculated using hematocrit $(\mathrm{Ht})$ and hemoglobin $(\mathrm{Hb})$ values $^{16}$.

RBC deformability was measured based on the ektacytometric principle using a Laser-assisted Optical Rotational Cell Analyser, LORCA (R\&R Mechatronics, Hoom, The Netherlands) ${ }^{17}$. Briefly, $25 \mu$ of EDTA-anticoagulated blood were suspended in $5 \mathrm{ml}$ of a $5 \%$-solution of polyvinylpyrrolidone ( $300 \mathrm{mOsm} / \mathrm{l}$, Sigma Chemical Company, Saint Louis, MO, USA). This sample was then subjected to several rotational speeds at $21{ }^{\circ} \mathrm{C}$, giving final shear stresses ranging from 0.30 to 53.35 Pascal $(\mathrm{Pa})$. Laser diffraction was used to follow the change of the RBC population from biconcave towards an ellipsoid morphology under increased shear stress, and the deformability (elongation index, El) of the RBC was calculated from the major and minor axis of the ellipsoid diffraction pattern (Figure 3.1). Apart from the near-maximum values at $30 \mathrm{~Pa}$ as previously used for evaluation of RBC deformability ${ }^{12,17,18}$, we also report values at $0.95,3$ and $9.5 \mathrm{~Pa}$, since, for example, rigidification of $\mathrm{RBC}$ with glutaraldehyde decreases the elongation index only at lower shear stresses ${ }^{18}$. The rest of the EDTAblood was centrifuged for 10 minutes at $3000 \mathrm{~g}$ and room temperature, and the viscosity of the plasma was measured in duplicate at a shear rate of $70 / \mathrm{s}$ using a 
Contraves LS 30 viscometer (Contraves, Zürich, Switzerland).

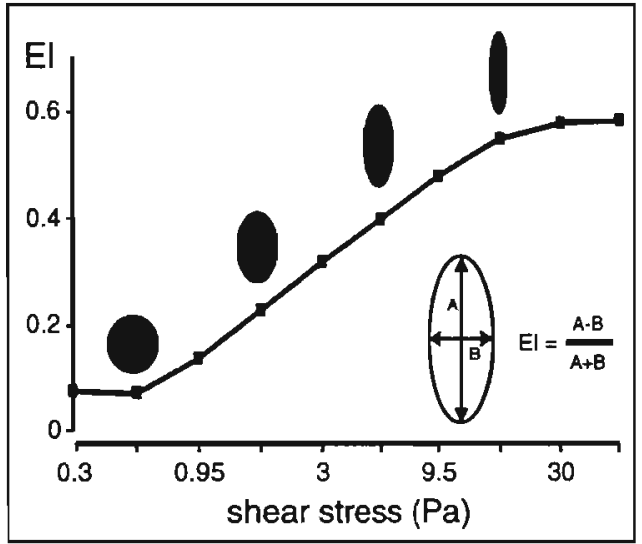

Figure 3.1. Change in diffraction pattern during elongation of RBC exposed to various shear stresses in vitro (log scale). The elongation index $(E I)$ is calculated from the major (A) and minor $(B)$ axis of the ellipsoid diffraction pattern.

For measurement of the osmotic fragility of RBC, each week 16 solutions ranging from $0.70 \%$ to $0.10 \%$ were prepared from a $1 \%$ salt solution $(9.0 \mathrm{~g} \mathrm{NaCl} / 1,1.71 \mathrm{~g}$ $\mathrm{Na}_{2} \mathrm{HPO}_{4} \cdot 2 \mathrm{H}_{2} \mathrm{O} / \mathrm{l}, 0.24 \mathrm{~g} \mathrm{NaH}_{2} \mathrm{PO}_{4} \cdot 2 \mathrm{H}_{2} \mathrm{O} / \mathrm{l}, \mathrm{pH} 7.4$ ), and stored for a maximum of 5 days at $4{ }^{\circ} \mathrm{C}$. To $5 \mathrm{ml}$ of each solutions, $25 \mu$ l of fresh heparinized blood were added, and carefully mixed. The samples were then left standing for $30 \mathrm{~min}$ at room temperature, carefully mixed again, and centrifuged for $10 \mathrm{~min}$ at $600 \mathrm{~g}$. The degree of hemolysis (osmotic fragility) was determined by measuring the release of $\mathrm{Hb}$ in the supematant spectrophotometrically at $540 \mathrm{~nm}$. Hemolysis in the $0.10 \%$ salt solution was considered as $100 \%$. Osmotic fragility in 5 salt solutions, demonstrating approximately 5 to $90 \%$ hemolysis, was used for interpretation of the results.

\section{Red blood cell phospholipid fatty acids}

RBC were washed twice with EDTA-containing saline (28.64 g NA ${ }_{2} E_{D T A} 2 \mathrm{H}_{2} \mathrm{O} /$, $7.00 \mathrm{~g} \mathrm{NaCl} / \mathrm{l}$ ), and stored under nitrogen at $-40^{\circ} \mathrm{C}$ for a maximum of 4 days. After thawing, phospholipids fatty acids were extracted from RBC, and analyzed by gas chromatography as described by Foreman-van Drongelen ${ }^{19}$, with the modification that fatty acids were separated on a BPX70 polar and a BP1 non-polar capillary column (Scientific Glass Engineering, Austin, TX, USA). Fish oil from the capsules was directly methylated with triheptadecanoin as intemal standard. The fatty acid composition of RBC phospholipids was expressed as weight percentage of total fatty 
acids $(g / g * 100 \%)$, and the polyunsaturated fatty acid (PUFA) unsaturation index as the sum of the molar percentage of each PUFA ( $\mathrm{mol} / \mathrm{mol}$ total fatty acids ${ }^{*} 100 \%$ ), multiplied with its number of double bonds.

\section{Plasma antioxidants}

EDTA-plasma was analyzed for retinol, tocopherols and carotenoids by HPLC with simultaneous absorbance and fluorescence detection as described by Hess et al..$^{20}$, with the modification that an intemal standard (retinol acetate) was used, and extraction of lipids was performed twice to improve recovery. Also, no ammonium acetate was added to the mobile phase. All samples from one subject were analyzed in the same analytical run, and the same number of subjects from each group was present in each run. Chromatogram peak areas were calculated with the Gynkosoft Chromatography Data System (Gynkotek, Germany), and calibrated against a mixture of the various standard substances dissolved in ethanol/dioxane/acetonitrile (1:1:3). Since pure phytofluene was not available, quantitative determination of this compound was not possible. B-Tocopherol co-eluted with $\gamma$-tocopherol, and they are therefore reported together. The canthaxanthin standard eluted separately from the lutein standard, but in plasma samples canthaxanthin concentrations were too low to be chromatographically separated from the lutein peak. Therefore, concentrations of lutein reported here may also include small traces of canthaxanthin.

Fish oil capsules were dissolved in ethanol/dioxane/acetonitrile $(20 \mathrm{mg} \mathrm{oil} / \mathrm{ml})$, and directly analyzed for tocopherols by HPLC.

$\alpha$-Tocopherol (all-rac), $\gamma$-tocopherol, B-cryptoxanthin, lycopene, and B-carotene standards were a generous gift from F. Hoffmann-La Roche (Basel, Switzerland). Lutein, retinol (all-trans), $\delta$-tocopherol and $\alpha$-carotene were obtained from Sigma Chemical Company, canthaxanthin from Fluka Chemie (Bomem, Belgium). Retinyl acetate and all other chemicals were purchased from Merck (Darmstadt, Germany).

\section{$L D L$ isolation and oxidation in vitro}

LDL was isolated from fresh EDTA-plasma immediately after collection by short-run single spin density gradient ultracentrifugation. In a $4.9 \mathrm{ml}$ OptiSeal polyallomer centrifuge tube (Beckman instruments, Palo Alto, CA, USA), $0.85 \mathrm{ml}$ of a $0.49 \mathrm{~g} / \mathrm{ml}$ potassium bromide solution and $1.52 \mathrm{ml}$ of plasma were gently mixed with a spatula. This mixture was carefuily overlayered with distilled water, containing $1 \mathrm{~g} / \mathrm{l}$ 
$\mathrm{Na}_{2}$ EDTA. $2 \mathrm{H}_{2} \mathrm{O}$, to the neck of the tube. The tube was then plugged, and centrifuged for 1 hour at $80,000 \mathrm{rpm}$ using a near vertical tube rotor (NVT-90, Beckman Instruments) in an XL-80 ultracentrifuge (Beckman Instruments). The upper $0.6 \mathrm{ml}$ was removed, and the next $1.6 \mathrm{ml}$, containing $L D L$, was collected. EDTA was removed from LDL samples by gel filtration, using two PD-10 Sephadex G25-M gel filtration columns (Pharmacia, Roosendaal, The Netherlands) placed on top of each other, and a nitrogen-purged mobile phase (PBS: $9.61 \mathrm{mmol} / / \mathrm{Na}_{2} \mathrm{HPO}_{4}, 1.56 \mathrm{mmol} / \mathrm{NaH} \mathrm{NaO}_{4}$, $0.154 \mathrm{~mol} / \mathrm{NaCl}, \mathrm{pH}$ 7.4). The EDTA-free LDL, was kept under a nitrogen atmosphere to prevent oxidation, and analyzed for its cholesterol content (Monotest Cholesterol, Boehringer Mannheim, Mannheim, Germany).

Within 15 minutes after gel filtration, an aliquot of each sample was diluted in a quartz cuvette with PBS (not purged with nitrogen) to a final concentration of 0.26 mmol cholesterol/, and oxidation was initiated with $\mathrm{CuCl}_{2}$ (f.c. $2 \mu \mathrm{mol} / \mathrm{l}$ ). Immediately thereafter a second aliquot of $\mathrm{LDL}(0.26 \mathrm{mmol} / \mathrm{l})$ was oxidized with $15 \mu \mathrm{mol} / / \mathrm{CuCl}_{2}$, since at copper/cholesterol ratios over $1 / 20(\mathrm{~mol} / \mathrm{mol})$ the copper concentration does not affect LDL oxidation parameters anymore (unpublished observation). Oxidation of PUFA at $37^{\circ} \mathrm{C}$ was measured spectrophotometrically by monitoring the formation of conjugated fatty acid dienes at $234 \mathrm{~nm}$. The lagtime before rapid formation of conjugated dienes was calculated from the intercept of linear lines through the point of maximum rate of diene formation and the absorbance immediately after addition of copper $^{21}$. In addition, the time at which the maximum rate of oxidation was reached $\left(T R_{m}\right)$ was also determined. $T R_{m}$ represents a combined effect of the lagtime and the rate of oxidation.

\section{Statistics}

Effects of endurance exercise were examined before supplementation for all 24 subjects together by Student's paired two-tailed $t$-test. To evaluate the effects of the supplements, responses were calculated for each subject as the changes in exercise parameters and pre-endurance exercise biochemical values over the period of supplementation. Differences in responses between the three experimental groups were then compared by analysis of variance (ANOVA). The placebo group allowed us to correct for possible drifts with time. Differences between the experimental groups 
in changes during exercise after supplementation as compared with changes during exercise before supplementation, were also compared by ANOVA. Pearson correlation coefficients ( $r$ ) were calculated to evaluate the relationship between RBC deformability and RBC osmotic fragility. Possible interference of the method of measurement of $\mathrm{ABC}$ deformability by changes in blood cell concentrations during exercise was also studied by computing Pearson correlation coefficients. The overall level of significance was set at $P<0.05$. As between-group ANOVA involved three simultaneous comparisons, between group levels of significance were set to $P<0.017$, according to the Bonferroni method. All statistical analyses were performed using the Statistical Analysis System (SAS Institute Inc., 1989). Values are reported as means \pm SEM. Although responses to exercise or supplementation may be given as percentages change, statistics were always performed on absolute changes.

\section{RESULTS}

\section{Effects of endurance exercise before supplementation}

Body weights (mean, $73 \mathrm{~kg}$; range, 64 to $92 \mathrm{~kg}$ ) were similar in all groups throughout the study. During the pre-supplementation endurance exercise test, subjects lost on average $0.56 \pm 0.07 \mathrm{~kg}$ (P $\leq 0.0001)$ of body weight, and consumed on average $0.65 \pm 0.06$ liters of water.

Effect of endurance exercise on hematological variables, plasma viscosity, and RBC characteristics

During the pre-supplementation endurance exercise test, the average RBC concentration, total $\mathrm{Hb}$, and $\mathrm{Ht}$ increased by 5.3 to $5.7 \%$. The mean corpuscular volume (MCV) and mean corpuscular hemoglobin concentration (MCHC) of the RBC did not change. White blood cell (WBC) concentrations increased from $5.38 \pm 0.16$ ${ }^{*} 10^{9} / \mathrm{l}$ before exercise to $7.48 \pm 0.29 * 10^{9} / \mathrm{l}$ after exercise, an average increase of $39 \%$ $(P \leq 0.0001)$. Platelet concentrations increased by $29 \%$, from $202 \pm 7^{*} 10^{9} / /$ to $259 \pm 10$ ${ }^{*} 10^{9} / /(\mathrm{P} \leq 0.0001)$. Blood volume decreased by $5.0 \pm 0.6 \%$ during exercise, and plasma volume by $9.0 \pm 1.0 \%$. 
Table 3.1. Effect of endurance exercise on red blood cell characteristics and the modification by fish oil and vitamin E supplementation

\section{AFTER SUPPLEMENTATION}

\section{BEFORE SUPPLEMENTATION}

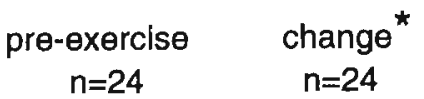

\begin{tabular}{|c|c|}
\hline PLA & \\
\hline $\begin{array}{c}\text { e-exercise } \\
n=8\end{array}$ & $\begin{array}{c}\text { change } \\
n=8\end{array}$ \\
\hline
\end{tabular}

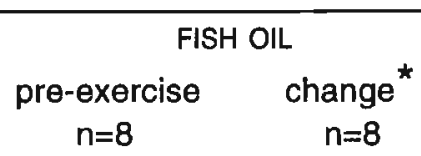

$\begin{array}{cc}\text { FISH OIL + VITAMIN E } \\ \begin{array}{c}\text { pre-exercise } \\ n=8\end{array} & \begin{array}{c}\text { change } \\ n=8\end{array}\end{array}$

\section{Deformability of red blood cells}

shear $(\mathrm{Pa})$

0.95

$0.129 \pm 0.002-0.005 \pm 0.003$

3

$0.319 \pm 0.004-0.005 \pm 0.002 \dagger$

9.5

$0.490 \pm 0.002$

$-0.002 \pm 0.002$

$0.582 \pm 0.002$

$0.139 \pm 0.008$
$0.334 \pm 0.010$
$0.503 \pm 0.004$
$0.586 \pm 0.002$

elongation index (EI)

$\begin{array}{lll}-0.002 \pm 0.005 & 0.138 \pm 0.007 & -0.007 \pm 0.003 \\ -0.005 \pm 0.007 & 0.332 \pm 0.007 & -0.009 \pm 0.002 \\ -0.003 \pm 0.003 & 0.504 \pm 0.004 & -0.009 \pm 0.002 \\ +0.002 \pm 0.002 & 0.587 \pm 0.002 & -0.002 \pm 0.002\end{array}$

$0.132 \pm 0.006+0.005 \pm 0.004$

$0.326 \pm 0.006+0.004 \pm 0.004$

$0.497 \pm 0.004+0.002 \pm 0.003$

$0.585 \pm 0.003+0.001 \pm 0.001$

Osmotic fragility of red blood cells salt solution
$0.52 \%$
$3.1 \pm 0.5$
$0.49 \%$
$17.3 \pm 1.6$
$+0.3 \pm 0.3$
$0.47 \%$
$37.0 \pm 2.8$
$+1.3 \pm 1.0$
$0.45 \%$
$68.5 \pm 2.3$
$+1.1 \pm 1.9$
$0.43 \%$
$87.4 \pm 1.2$
$+0.9 \pm 1.4$
$+0.3 \pm 0.7$

hemolysis (\%)

$\begin{array}{rr}5.8 \pm 2.6 & +0.7 \pm 0.5 \\ 24.2 \pm 6.5 & +2.2 \pm 1.2 \\ 41.7 \pm 8.3 & +2.7 \pm 2.2 \\ 68.1 \pm 6.3 & +2.1 \pm 2.8 \\ 84.5 \pm 3.3 & +1.8 \pm 1.4\end{array}$

$$
\begin{array}{r}
4.1 \pm 0.9 \\
22.7 \pm 4.3 \\
43.0 \pm 5.1 \\
74.0 \pm 3.9 \\
90.9 \pm 3.3
\end{array}
$$

$\begin{aligned} 4.8 & \pm 0.9 \\ 24.5 & \pm 3.6 \\ 45.8 & \pm 4.4 \\ 76.3 & \pm 2.6 \\ 90.9 & \pm 0.9\end{aligned}$

$+1.3 \pm 0.4$

$+4.7 \pm 1.3$

$+4.2 \pm 2.5$

$+3.0 \pm 1.2$

$+1.3 \pm 0.4$

Subjects performed a time trial of approximately 1 hour on a bicycle ergometer, before and after supplementation with placebo, fish oil (6 g/day) or fish oil (6 g/day) plus vitamin E (300 IU/day) for three weeks. Deformability and osmotic fragility of red blood cells were measured as described in "subjects and methods". Values are means \pm SEM.

* Before supplementation, changes during exercise were examined by Student's paired $t$-test: $\dagger, P<0.05$. After supplementation, changes during exercise in the 3 groups were compared by ANOVA, but no significant differences in change were noticed between the groups. 
Plasma viscosity increased from $1.46 \pm 0.02 \mathrm{mPa}$.s before exercise to $1.52 \pm 0.02$ $\mathrm{mPa} \cdot \mathrm{s}$ after exercise, an average increase of $4.2 \%(\mathrm{P}=0.005)$.

RBC deformability decreased during exercise (Table 3.1), but only the $1.6 \%$ decrease at a shear rate of $3 \mathrm{~Pa}$ reached statistical significance $(P=0.035)$. The decreases in deformability correlated with increases in RBC concentration (e.g. at 3 $\mathrm{Pa}: r=-0.494, \mathrm{P}=0.014$; at $30 \mathrm{~Pa}: r=-0.454, \mathrm{P}=0.026$ ), but not with increases in WBC concentration, platelet concentration, or plasma viscosity. Although the changes in deformability also correlated with changes in plasma volume ( $3 \mathrm{Pa:} r=0.455, P=0.025$; $30 \mathrm{~Pa}: r=0.410, P=0.047$ ), correlations with changes in blood volume ( $3 \mathrm{~Pa}: r=0.334$, $\mathrm{P}=0.110 ; 30 \mathrm{~Pa}: r=0.385, \mathrm{P}=0.064$ ) did not reach statistical significance.

The osmotic fragility of RBC (\% hemolysis) was not significantly affected by endurance exercise (Table 3.1). Interestingly, a high RBC deformability at low shear stresses $(0.95,3,9.5 \mathrm{~Pa})$, but not at $30 \mathrm{~Pa}$, was generally associated with a high osmotic fragility. For example, Pearson correlation coefficients of the osmotic fragility in a $0.47 \%$ salt solution versus $\mathrm{RBC}$ deformability at $3 \mathrm{~Pa}$ and $30 \mathrm{~Pa}$ were 0.477 $(P=0.018)$ and $0.002(P=0.992)$, respectively.

\section{Effect of endurance exercise on plasma antioxidants}

Plasma antioxidant concentrations, except for $\delta$-tocopherol and $B+\gamma-$ tocopherol, increased significantly $(P \leq 0.0001)$ during endurance exercise. Retinol concentrations increased by $10.1 \pm 0.7 \%$, $\alpha$-tocopherol concentrations by $7.2 \pm 0.7 \%$, and total carotenoid concentrations by $4.8 \pm 0.9 \%$. After correction for the decrease in plasma volume in each subject, however, plasma antioxidant concentrations decreased significantly, except for retinol (Table 3.2). Total tocopherol concentrations decreased by $2.9 \pm 1.1 \% \quad(P=0.012)$, and total carotenoid concentrations by $4.5 \pm 1.2 \%$ $(\mathrm{P}=0.0008)$.

Effect of endurance exercise on LDL oxidation in vitro

LDL was more rapidly oxidized in vitro by the high copper concentration, as compared with the low copper concentration. However, at both concentrations, the lagtime before onset of rapid oxidation of LDL in vitro, the maximum rate of oxidation, and the time at which this maximum was reached $\left(T_{m}\right)$ were not affected by endurance exercise. 
Table 3.2. Effect of endurance exercise on plasma antioxidant concentrations, and the modification by fish oil and vitamin $\mathrm{E}$ supplementation

\section{AFTER SUPPLEMENTATION}

BEFORE SUPPLEMENTATION

$\underset{n=24}{\text { pre-exercise change }}$ *
PLACEBO

pre-exercise $n=8$
FISH OIL
pre-exercise

change *

$$
n=8
$$

$1.77 \pm 0.11$

$-0.043 \pm 0.026$
FISH OIL + VITAMIN E pre-exercise change ${ }^{*}$ $n=8$ $n=8$

\begin{tabular}{|c|c|c|c|c|c|c|c|c|c|}
\hline Retinol & $1.82 \pm 0.06$ & $+0.004 \pm 0.020$ & & $1.77 \pm 0.11$ & $-0.043 \pm 0.026$ & $2.09 \pm 0.09$ & $+0.011 \pm 0.025$ & $1.83 \pm 0.11$ & $+0.031 \pm 0.016$ \\
\hline Tocopherols & $25.1 \pm 1.0$ & $-0.77 \pm 0.28$ & $t$ & $26.4 \pm 2.2$ & $-1.29 \pm 0.52$ & $27.1 \pm 1.7$ & $-0.26 \pm 0.23$ & $36.4 \pm 2.6$ & $-0.17 \pm 0.42$ \\
\hline$\delta$-tocopherol & $0.15 \pm 0.01$ & $-0.011 \pm 0.004$ & $\dagger$ & $0.17 \pm 0.03$ & $0.022 \pm 0.009$ & $0.18 \pm 0.02$ & $-0.015 \pm 0.008$ & $0.09 \pm 0.01$ & $0.000 \pm 0.001$ \\
\hline$B+\gamma$-tocopherol & $2.19 \pm 0.19$ & $-0.162 \pm 0.035$ & $\S$ & $2.79 \pm 0.44$ & $0.283 \pm 0.097$ & $.40 \pm 0.30$ & $-0.121 \pm 0.028$ & $.75 \pm 0.09$ & $-0.026 \pm 0.014$ \\
\hline$\alpha$-tocopherol & $22.7 \pm 0.9$ & $-0.60 \pm 0.26$ & $\dagger$ & $23.4 \pm 1.9$ & $-0.98 \pm 0.42$ & $24.5 \pm 1.6$ & $-0.12 \pm 0.22$ & $35.5 \pm 2.5$ & $-0.15 \pm 0.42$ \\
\hline aly & 1.89 & $-0.082 \pm 0$ & $\S$ & & & & & & \\
\hline lute & $0.51 \pm 0.02$ & $-0.026 \pm 0.006$ & $\S$ & $0.58 \pm 0.06$ & -0.0 & 07 & $-0.016 \pm 0$ & $0.47 \pm$ & $05 \pm 0$ \\
\hline B-cryp & $0.32 \pm c$ & $-0.014 \pm 0.005$ & $\ddagger$ & & & & $05 \pm 0$. & & 002 \\
\hline Iycopene & $0.61 \pm 0.06$ & $-0.022 \pm 0.007$ & $\ddagger$ & $0.62 \pm 0.14$ & $-0.044 \pm 0.028$ & $0.53 \pm 0.05$ & $-0.012 \pm 0.011$ & $0.47 \pm 0.07$ & $-0.007 \pm 0.005$ \\
\hline$\alpha$-carotene & $0.07 \pm 0.01$ & $-0.003 \pm 0.001$ & $\ddagger$ & $0.06 \pm 0$ & $-0.003 \pm c$ & 01 & $-0.001 \pm 0$ & 22 & $03 \pm 0.001$ \\
\hline B-carotene & $0.38 \pm 0.03$ & $-0.017 \pm 0.004$ & $\S$ & $0.35 \pm 0.04$ & $-0.018 \pm 0.008$ & $0.41 \pm 0.03$ & $-0.006 \pm 0.010$ & $0.42 \pm 0.14$ & $-0.010 \pm 0.005$ \\
\hline 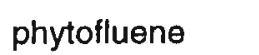 & $0.58 \pm 0.07$ & $-0.033 \pm 0.009$ & $\S$ & $0.65 \pm 0.19$ & $-0.060 \pm 0.038$ & $0.52 \pm 0.04$ & $-0.018 \pm 0.012$ & $0.46 \pm 0.12$ & $-0.014 \pm 0.006$ \\
\hline
\end{tabular}

Subjects performed a time trial before and after supplementation (for details, see Table 3.1 and text). Antioxidant concentrations are expressed in $\mu \mathrm{mol} / \mathrm{l}$ plasma. For phytofluene, chromatogram peak area are expressed in $\mathrm{mV} \times \mathrm{min} / \mu$ l plasma (amplificatlon 100). Values are means $\pm \mathrm{SEM}$.

* Changes during exercise are adjusted for changes in plasma volume in each subject. Before supplementation, changes during exercise were examined by Student's paired $t$-test: $† P<0.05, \ddagger P<0.01, \S P<0.001$. After supplementation, changes during exercise in the 3 groups were compared by ANOVA, but no significant differences in change were noticed between the groups. 
Table 3.3. Effect of endurance exercise on oxidation of LDL in vitro, and the modification by fish oil and vitamin $E$ supplementation

AFTER SUPPLEMENTATION

BEFORE SUPPLEMENTATION

\begin{tabular}{|c|c|}
\hline pre-exercise & change ${ }^{\star}$ \\
\hline$n=24$ & $n=24$ \\
\hline
\end{tabular}

$\begin{array}{cc}\text { PLACEBO } \\ \text { pre-exercise } \\ n=8 & \text { change } \\ & n=8\end{array}$

\begin{tabular}{|c|c|}
\hline \multicolumn{2}{|c|}{ FISH OIL } \\
\hline pre-exercise & change * \\
\hline$n=8$ & $n=8$ \\
\hline
\end{tabular}

\begin{tabular}{|c|c|}
\hline \multicolumn{2}{|c|}{ FISH OIL + VITAMIN E } \\
\hline $\begin{array}{c}\text { pre-exercise } \\
n=8\end{array}$ & $\begin{array}{c}\text { change } \\
n=8\end{array}$ \\
\hline
\end{tabular}

Oxidation induced with $2 \mu \mathrm{mol} / \mathrm{l} \mathrm{CuCl}_{2}$

Lagtime

$\mathrm{A}_{\text {max }}$

$66.4 \pm 1.8$

$3.48 \pm 0.08$

$-0.5 \pm 0.9$

$T_{\mathrm{m}}$

$95.0 \pm 2.1$

$-0.06 \pm 0.05$

Dienes
$-0.5 \pm 1.1$

$-2.1 \pm 1.0^{\dagger}$

$\begin{array}{rr}65.3 \pm 3.5 & +3.4 \pm 1.9 \\ 3.48 \pm 0.06 & -0.02 \pm 0.03 \\ 94.0 \pm 3.9 & +4.0 \pm 1.9 \\ 156.6 \pm 3.5 & -1.1 \pm 1.4\end{array}$

$\begin{array}{rrr}59.7 \pm 2.0 & -0.1 \pm 1.8 \\ 3.02 \pm 0.10 & +0.13 \pm 0.09 \\ 93.0 \pm 2.2 & -0.9 \pm 2.9 \\ 159.2 \pm 4.0 & -1.4 \pm 0.6\end{array}$

$75.9 \pm 4.0$

$2.66 \pm 0.09$

$114.8 \pm 5.0$

$162.1 \pm 3.6$
$-0.7 \pm 1.8$

$+0.02 \pm 0.06$

$-3.4 \pm 2.9$

$-3.9 \pm 1.6$

Oxidation induced with $15 \mu \mathrm{mol} / / \mathrm{CuCl}_{2}$

\begin{tabular}{lrrrrrrrrrr} 
Lagtime & $59.0 \pm 1.8$ & $+1.4 \pm 1.6$ & $56.0 \pm 6.3$ & $+4.8 \pm 5.0$ & $52.2 \pm 3.6$ & $-2.4 \pm 1.7$ & $65.1 \pm 2.8$ & $+1.6 \pm 3.9$ \\
$\mathrm{R}_{\max }$ & $5.00 \pm 0.18$ & $-0.18 \pm 0.20$ & $5.24 \pm 0.36$ & $-0.28 \pm 0.45$ & $4.46 \pm 0.34$ & $+0.20 \pm 0.22$ & $3.92 \pm 0.28$ & $-0.41 \pm 0.24$ \\
$\mathrm{TR}_{\mathrm{m}}$ & $78.3 \pm 2.2$ & $+2.0 \pm 2.3$ & $73.9 \pm 7.3$ & $+5.0 \pm 6.0$ & $73.9 \pm 4.5$ & $-3.3 \pm 2.6$ & $91.4 \pm 3.8$ & $+2.4 \pm$ & 5.0 \\
Dienes & $154.4 \pm 2.2$ & $-2.4 \pm 1.1$ & $153.7 \pm 3.2$ & $-1.6 \pm 1.4$ & $152.3 \pm 3.0$ & $-2.2 \pm 1.4$ & $157.9 \pm 3.2$ & $-4.6 \pm 1.9$ \\
\hline
\end{tabular}

Subjects performed a time trial before and after supplementation (for details, see Table 3.1 and text). LDL (0.26 mmol cholesterol/l) was oxidized in vitro with 2 and $15 \mu \mathrm{mol} \mathrm{CuCl} / 1$ at $37^{\circ} \mathrm{C}$, and the formation of conjugated dienes was monitored spectrophotometrically at $234 \mathrm{~nm}$. Lagtime before onset of rapid oxidation and the time at which the maximum rate of diene formation was reached $\left(T R_{m}\right)$ are expressed in minutes, the maximum rate $\left(R_{m a x}\right)$ in $\mu \mathrm{mol} / \mathrm{mmol} \mathrm{LDL}$ cholesterol $/ \mathrm{min}$, and the amount of conjugated dienes formed in $\mu \mathrm{mol} / \mathrm{mmol} L D L$ cholesterol. Values are means \pm SEM.

* Before supplementation, changes during exercise were examined by Student's paired $t$-test: $† P<0.05$. After supplementation, changes during exercise in the 3 groups were compared by ANOVA, but no significant differences in change were noticed between the groups. 
The maximum amount of conjugated dienes formed during low-copper oxidation in vitro decreased by $2.1 \pm 1.0 \mathrm{nmol} / \mu \mathrm{mol} L D L$ cholesterol $(\mathrm{P}=0.042)$ during the presupplementation endurance exercise test. A similar decrease was noticed in LDL oxidized with the high copper concentration (Table 3.3).

\section{Effects of fish oil and vitamin E supplementation}

\section{Effect of supplementation on RBC phospholipid fatty acids}

Compliance to the fish oil supplements was confimed by an increase in the proportion of $n-3$ PUFA in RBC from $5.2 \%$ to $7.0 \%$ in the FO group (P $\leq 0.0001$ vs PLA) and from $5.2 \%$ to $7.6 \%$ in the FE group ( $P \leq 0.0001$ vs $P L A$ ) during the study (Table 3.4). The increase in EPA was larger in the FE group ( $P=0.001$ vs $F O$ ), but other indicators of compliance, like changes in DHA and intake of capsules, were similar. Although increases in n-3 PUFA were accompanied by decreases in n-6 PUFA, the degree of unsaturation was significantly increased after supplementation with fish oil (Table 3.4).

\section{Effect of supplementation on maximal workload capacity}

Pre-stratification by $W_{\max }$ at the start of the study resulted in similar values of $5.0 \pm 0.2,4.9 \pm 0.2,5.0 \pm 0.2$ watt $/ \mathrm{kg}$ in the PLA, FO, and FE group, respectively. During the study, $W_{\max }$ slightly improved in all groups $(0.12$ watt $/ \mathrm{kg}$ or $2.5 \%)$, but no significant differences between the groups were noticed.

At the start of the study, mean maximal oxygen consumption $\left(\mathrm{VO}_{2 \text { max }}\right)$ during the test was $4.4 \pm 0.1 \mathrm{~V} / \mathrm{min}$, and did not differ significantly between the groups. After supplementation, $\mathrm{VO}_{2 \max }$ increased by $0.01 \pm 0.09 \mathrm{Vmin}$ in the PLA, by $0.13 \pm 0.13$ $\mathrm{V} / \mathrm{min}$ in the FO group, and by $0.15 \pm 0.09 \mathrm{~V} / \mathrm{min}$ in the FE group. However, these changes were not significantly different between the groups $(P>0.3)$.

After PLA and FE supplementation, lactate concentrations appeared to increase less rapidly during the $W_{\max }$ test, as compared with the test at the start of the study. In the PLA group, lactate concentrations of $4 \mathrm{mmol} / \mathrm{l}$ were reached at a workload of $3.77 \pm 0.17$ watt $/ \mathrm{kg}$ during the first test, and at $4.06 \pm 0.15$ watt $/ \mathrm{kg}$ during the second test (change: $+0.29 \pm 0.08$ watt $/ \mathrm{kg} ; \mathrm{P}=0.022$ vs $\mathrm{FO}$ ). In the $\mathrm{FE}$ group these values were $3.77 \pm 0.22$ and $3.99 \pm 0.15$, respectively $(+0.22 \pm 0.08$ watt $/ \mathrm{kg} ; P=0.074$ vs $F O)$. 
Table 3.4. Fatty acid composition of the fish oil concentrate and the effects on the fatty acid composition of red blood cell phospholipids after three weeks of fish oil supplementation

Red blood cell phospholipid fatty acids in experimental groups

\begin{tabular}{|c|c|c|c|c|c|c|c|c|}
\hline \multirow{3}{*}{$\begin{array}{c}\text { Fish oil } \\
\text { concentrate }\end{array}$} & \multirow{2}{*}{\multicolumn{2}{|c|}{ PLACEBO }} & \multirow{2}{*}{\multicolumn{3}{|c|}{ FISH OIL }} & \multirow{2}{*}{\multicolumn{3}{|c|}{ FISH OIL + VITAMIN E }} \\
\hline & & & & & & & & \\
\hline & baseline & change & baseline & change & & baseline & change & \\
\hline \multicolumn{9}{|c|}{ grams per 100 grams fatty acids } \\
\hline 25.9 & $47.9 \pm 0.3$ & $-0.4 \pm 0.2$ & $47.4 \pm 0.2$ & $+0.2 \pm 0.2$ & & $47.7 \pm 0.3$ & $-0.2 \pm 0.3$ & \\
\hline 26.5 & $17.8 \pm 0.2$ & $-0.1 \pm 0.2$ & $18.2 \pm 0.4$ & $-0.2 \pm 0.1$ & & $17.7 \pm 0.3$ & $-0.1 \pm 0.1$ & \\
\hline 39.7 & $33.5 \pm 0.2$ & $+0.4 \pm 0.1$ & $33.4 \pm 0.4$ & $+0.2 \pm 0.1$ & & $33.7 \pm 0.2$ & $+0.2 \pm 0.2$ & \\
\hline 3.8 & $28.4 \pm 0.2$ & $+0.3 \pm 0.1$ & $28.0 \pm 0.4$ & $-1.7 \pm 0.2$ & $\ddagger$ & $28.3 \pm 0.5$ & $-2.1 \pm 0.3$ & $\ddagger$ \\
\hline 1.2 & $12.4 \pm 0.4$ & $+0.2 \pm 0.2$ & $12.2 \pm 0.3$ & $-0.7 \pm 0.2$ & $\dagger$ & $13.2 \pm 0.4$ & $-1.3 \pm 0.2$ & $¥$ \\
\hline 1.1 & $10.7 \pm 0.2$ & $+0.1 \pm 0.1$ & $10.4 \pm 0.2$ & $-0.5 \pm 0.1$ & $\ddagger$ & $10.0 \pm 0.3$ & $-0.3 \pm 0.1$ & $t$ \\
\hline 35.7 & $4.9 \pm 0.2$ & $0.0 \pm 0.1$ & $5.2 \pm 0.2$ & $+1.8 \pm 0.1$ & $¥$ & $5.2 \pm 0.3$ & $+2.4 \pm 0.1$ & $\ddagger, \S$ \\
\hline 17.6 & $0.5 \pm 0.1$ & $0.0 \pm 0.0$ & $0.5 \pm 0.1$ & $+0.8 \pm 0.1$ & $\ddagger$ & $0.5 \pm 0.1$ & $+1.2 \pm 0.1$ & $\ddagger, \S$ \\
\hline 12.5 & $2.4 \pm 0.2$ & $0.0 \pm 0.0$ & $2.6 \pm 0.1$ & $+0.7 \pm 0.1$ & $\ddagger$ & $2.8 \pm 0.2$ & $+0.8 \pm 0.0$ & $\ddagger$ \\
\hline 7.9 & $1.0 \pm 0.1$ & $+0.1 \pm 0.1$ & $1.0 \pm 0.1$ & $0.0 \pm 0.1$ & & $1.0 \pm 0.1$ & $+0.1 \pm 0.1$ & \\
\hline n index ${ }^{\star}$ & $102.1 \pm 0.9$ & $+0.9 \pm 0.5$ & $102.4 \pm 0.8$ & $+3.5 \pm 0.6$ & $t$ & $102.0 \pm 0.6$ & $+5.3 \pm 0.4$ & $\ddagger$ \\
\hline
\end{tabular}

Twenty-four athletes received for three weeks a placebo, fish oil ( $6 \mathrm{~g} /$ day), or fish oil $(6 \mathrm{~g} /$ day) plus vitamin $\mathrm{E}$ ( $300 \mathrm{lU} /$ day) supplement to their habitual diets. Values for red blood cell phospholipid fatty acid composition are means \pm SEM. Changes after supplementation in the 3 groups were compared by ANOVA.

$t_{1} \neq$ Significant difference from change in placebo group: $\dagger P<0.01, \mp P \leq 0.0001$.

$\S$ Significant difference from change in fish oil group: $P<0.01$.

* Calculated as the sum of the percentage of each polyene (moles/moles total fatty acids ${ }^{*} 100 \%$ ) multiplied with its number of double bonds. 
In the FO group, however, lactate concentrations of $4 \mathrm{mmo} / /$ were reached at similar workloads in both tests $(3.87 \pm 0.20$ and $3.89 \pm 0.19$ watt $/ \mathrm{kg}$, respectively; change: $+0.01 \pm 0.08$ watt $/ \mathrm{kg}$ ). Since increases in lactate concentrations during the test may have slightly deviated from linearity around the $4 \mathrm{mmol} / \mathrm{l}$ point, we also looked at lactate concentrations at workloads just below (250 watt) and above ( 300 watt) this point. At 250 watt, lactate concentrations in the PLA group were $0.80 \pm 0.28$ ( $P=0.085$ vs $\mathrm{FO}$ ) and in the FE group $0.68 \pm 0.21$ ( $P=0.166$ vs $\mathrm{FO}$ ) $\mathrm{mmol} / \mathrm{l}$ lower during the second test as compared with the first test, whereas they were only $0.19 \pm 0.22$ $\mathrm{mmo} / / \mathrm{l}$ lower in the FO group. At 300 watt, these changes were $-1.23 \pm 0.41$ (PLA, $P=0.107$ vs $F O$ ), $-1.46 \pm 0.44$ (FE, $P=0.046$ vs $F O$ ), and $-0.32 \pm 0.28$ (FO) $\mathrm{mmol} /$.

\section{Effect of supplementation on endurance exercise performance}

The average time to complete the endurance exercise test at the start of the study was $55.9 \pm 0.6 \mathrm{~min}(n=24)$. After supplementation, performances were similar in all groups, and changed only by $+0.11 \pm 0.49,-0.23 \pm 1.35$, and $-0.07 \pm 1.16 \mathrm{~min}$ in the PLA, FO, and FE group, respectively ( $P>0.8$ between groups).

Effect of supplementation on hematological variables, plasma viscosity, and RBC characteristics

After supplementation, $\mathrm{RBC}$ concentrations, $\mathrm{Hb}, \mathrm{Ht}, \mathrm{MCV}$, and $\mathrm{MCHC}$ were similar to values before supplementation. Average changes over the 3 weeks were no more than $3 \%$. Plasma viscosity decreased by $0.05 \pm 0.03 \mathrm{mPa} \cdot \mathrm{s}$ in the PLA group, by $0.09 \pm 0.04 \mathrm{mPa} \cdot \mathrm{s}$ in the $\mathrm{FO}$ group, and by $0.02 \pm 0.03 \mathrm{mPa} \cdot \mathrm{s}$ in the FE group. Differences between treatments were not significant. Changes during exercise were also similar in the three groups.

RBC deformability increased slightly during the study. Atthough the increases during the study at lower stress rates appeared to be larger in the FO group, differences between treatments were not statistically significant. A decrease in RBC deformability during endurance exercise as seen before supplementation, was not present anymore in all groups after supplementation. Differences between the groups in changes during exercise, however, did not reach statistical significance (Table 3.1).

The osmotic fragility of RBC was not significantly affected by the supplements, nor did they induce changes during exercise (Table 3.1). 
Effect of supplementation on plasma antioxidants

Intake of the vitamin E capsules in the FE group was verified by an increase in the mean plasma $\alpha$-tocopherol concentration from $20.0 \pm 1.0$ to $35.5 \pm 2.5 \mu \mathrm{mol} / /$ ( $\mathrm{P} \leq 0.0001$ versus other groups). This was accompanied by a non-significant decrease in $\delta$-tocopherol concentrations, and a significant decrease in $\beta+\gamma$-tocopherol concentrations from $1.93 \pm 0.25$ to $0.75 \pm 0.09 \mu \mathrm{mol} / \mathrm{l}$ ( $P<0.004$ vs other groups). Before supplementation, retinol concentrations in the FO group were non-significantly higher than concentrations in the other groups. During the study, plasma retinol concentrations changed by $-0.02 \pm 0.05 \mu \mathrm{mol} / \mathrm{l}$ in the PLA group, by $+0.08 \pm 0.06$ $\mu \mathrm{mol} / \mathrm{l}$ in the $\mathrm{FO}$ group ( $\mathrm{P}=0.207 \mathrm{vs} P L A)$, and by $+0.15 \pm 0.06 \mu \mathrm{mol} / \mathrm{l}$ in the $\mathrm{FE}$ group $(P=0.041$ vs $P L A ; P=0.390$ vs $F O)$. Carotenoid concentrations were also not affected by the supplements.

Decreases in plasma antioxidant concentrations during endurance exercise were smaller after FO and FE supplementation, than exercise-induced decreases after PLA supplementation (Table 3.2). However, differences between the groups did not reach statistical significance.

\section{Effect of supplementation on $L D L$ oxidation in vitro}

Before supplementation, LDL oxidation parameters were similar in all 3 groups. At the end of the supplementation period, the lagtime during low-copper oxidation tended to increase in the FE group as compared with the other groups ( $P=0.052$ vs $F O$; $P=0.113$ vs $P L A ;$ Figure 3.2). The rate of oxidation in vitro, decreased by $0.52 \pm 0.16$ $\mathrm{nmol}$ dienes/mmol LDL cholesterol/min in the FO group ( $P=0.010$ vs $P L A$ ), and by $0.82 \pm 0.18 \mathrm{nmol} / \mathrm{mmol}$ in the FE group ( $P=0.0003$ vs $P L A ; P=0.159$ vs $F O$ ), whereas the PLA group showed a slight increase $(0.07 \pm 0.08 \mathrm{nmol} / \mathrm{mmol}$; Figure 3.2$)$. $\mathrm{TR}_{\mathrm{m}}$ remained unchanged in the PLA and FO group, but increased significantly in the FE group (change: $+15.0 \pm 5.7 ; P=0.007$ vs $P L A ; P=0.040$ vs FO; Figure 3.2). The maximum amount of dienes formed in vitro did not change significantly during the study, and no differences were therefore noticed between the groups (Figure 3.2). Similar results were found for oxidation of LDL with a high copper concentration (figure not shown).

After supplementation, changes in parameters of LDL oxidation during exercise, if any, did not differ significantly between the groups (Table 3.3). 


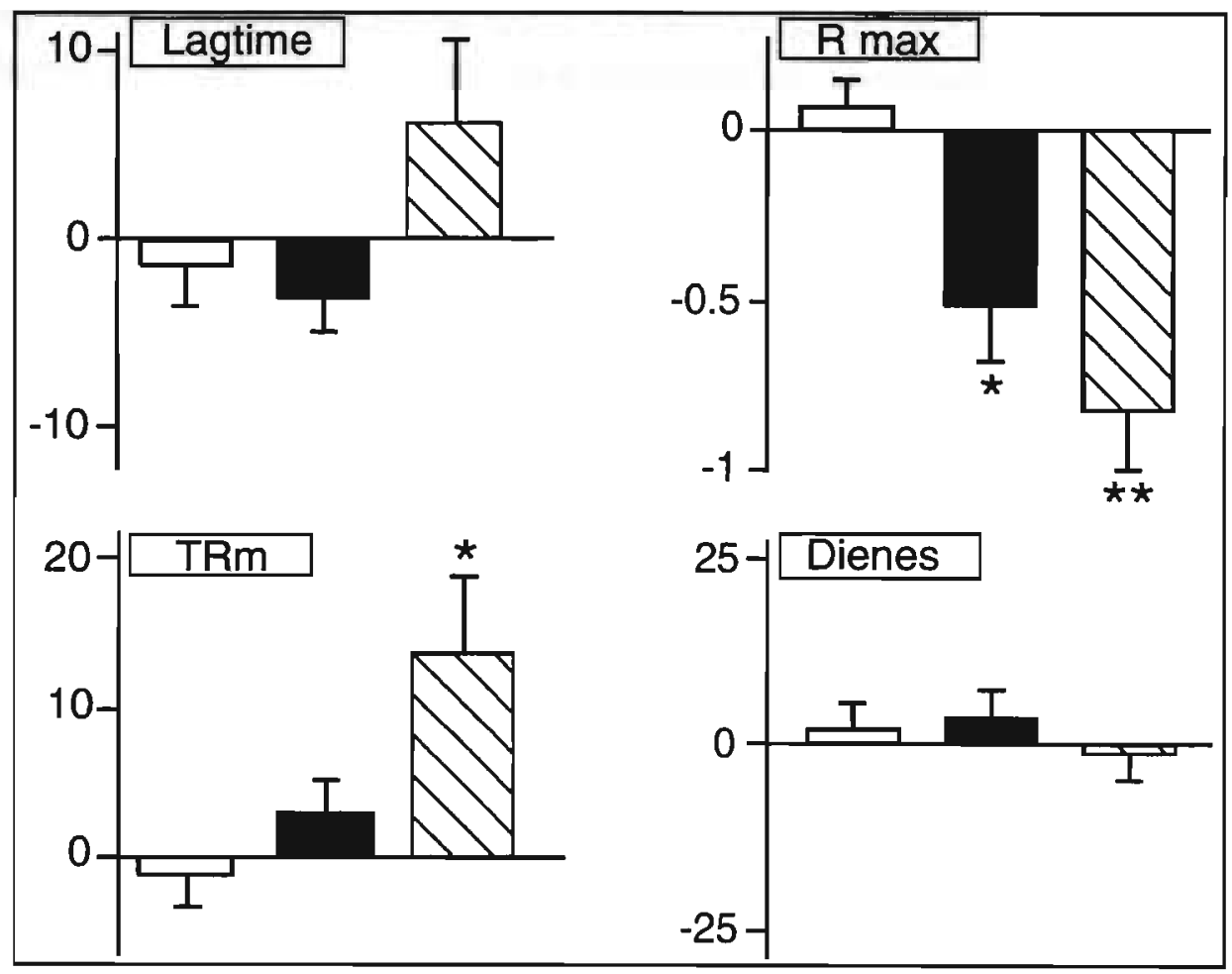

Figure 3.2. Effect of placebo (open bars), fish oil (solid bars), and fish oil plus vitamin $E$ (hatched bars) supplementation on oxidation of $\mathrm{LDL}$ in vitro catalyzed by $2 \mu \mathrm{mol} / \mathrm{l}$ of $\mathrm{CuCl}_{2}$. Bars indicate the mean change (\& SEM) after three weeks of supplementation. Lagtimes and the time of maximum rate $(T R m)$ are expressed in minutes, the maximum rate of oxidation (R max) in $\mathrm{nmol} / \mu \mathrm{mol} \mathrm{LDL}$ cholesterol/min, and the maximum amount of conjugated dienes in $\mathrm{nmol} / \mu \mathrm{mol} \mathrm{LDL}$ cholesterol. Asterisks indicate significant differences from change in placebo group (ANOVA): ", P $\leq 0.01 ; "{ }^{*}, \mathrm{P}<0.001$.

\section{DISCUSSION}

The present study examined the effects of fish oil and vitamin $E$ supplementation on exercise performance, RBC characteristics, plasma lipid-soluble antioxidant status, and in vitro LDL oxidation. 


\section{Effects of endurance exercise before supplementation}

Effect of endurance exercise on hematological variables and RBC characteristics

During endurance exercise a hemoconcentration of approximately $5 \%$ was observed, which was caused by a $9 \%$ reduction of the plasma compartment. At the same time, RBC deformability slightly decreased, but the osmotic fragility was not affected. The measured decrease in RBC deformability appeared to be related to hemoconcentration, although Hardeman et al. ${ }^{17}$ showed that the elongation index does not decrease with increasing numbers of RBC per $\mathrm{ml}$ of polyvinylpyrrolidone solution. The increases in WBC, platelets and plasma viscosity during exercise did not correlate with the decreases in RBC deformability, and it is therefore unlikely that these changes have affected the measurement of RBC deformability.

Van der Brug et al. ${ }^{12}$ measured the elongation index at $30 \mathrm{~Pa}$, and found a significant decrease of $0.7 \%$ after a combination of two cycling bouts and two running bouts, with an average total duration of $140 \mathrm{~min}$. After a $100-\mathrm{km} \mathrm{race}^{10}$, and after a marathon ${ }^{11}$, RBC deformability was also impaired, as measured by filterability of RBC and whole blood, respectively. Others, however, did not find a decrease in RBC deformability due to exercise as evaluated by RBC filterability after a marathon ${ }^{22}$ or by relative blood vs plasma viscosity at high shear rates after a 1-h cycling exercise ${ }^{23}$. Wood et al. ${ }^{24}$ even found an increase in relative viscosity after a 3 to 6 hour cross-country run, performed at an altitude of 1700-3000 m. Conversely, Guezennec et al..$^{25}$ found a decrease in RBC filterability during a 1 hour cycling exercise at a simulated altitude of $\mathbf{3 0 0 0} \mathrm{m}$ in a hypobaric chamber, but not at sea level. However, the high variability of filtration techniques and the possibility of analytical artefacts ${ }^{18,26}$ complicate comparison with the sensitive and reproducible technique used in the present study.

In the present study, the change in RBC deformability was small $(<2 \%)$, but together with the $4.2 \%$ increase in plasma viscosity may have a significant effect on whole blood viscosity, as demonstrated by van der Brug et al. ${ }^{12}$.

The unchanged osmotic fragility of RBC following exercise appears to be in conflict with results from Smith et $a l^{27}$, who reported a decrease. They suggested that this decrease was caused by RBC dehydration, as indicated by a decrease in MCV and an increase in RBC density. In the present study MCV did not change during exercise, however. 


\section{Effect of endurance exercise on plasma antioxidants}

Plasma antioxidant concentrations increased during exercise, but after correction for hemoconcentration, tocopherol and carotenoid concentrations decreased significantly.

Comparable to the present study, an increase in plasma vitamin $E$ was reported after 3 times of $15 \mathrm{~min}$ of running exercise ${ }^{28}$. However, correction for hemoconcentration neutralized this change. No changes in plasma vitamin $E$ concentration were found after a half-marathon ${ }^{29}$ or after 90 minutes of submaximal cycling ${ }^{30}$, but these data were not adjusted for hemoconcentration. A decrease in plasma vitamin $E$ is therefore conceivable. Duthie et al. ${ }^{29}$ found an $18 \%$ increase in plasma retinol concentration after a half-marathon, which was only partly explained by the $6 \%$ decrease in plasma volume. Antioxidant concentrations, however, may have been influenced by food intake during exercise, which was not allowed in our study.

It is unlikely that the loss of plasma tocopherols during exercise in the present study was caused by a transfer to $\mathrm{RBC}^{31}$, since it has been shown that $\mathrm{RBC}$ vitamin $E$ concentrations do not increase immediately after a half-marathon ${ }^{29}$. In addition, Nair et al. ${ }^{32}$ found no detectable concentrations of B-carotene in RBC. However, exercise-activated lipoprotein lipase ${ }^{33}$ may have caused a transfer of tocopherols ${ }^{34,35}$, and maybe also of carotenoids, from the circulation to tissues. This might also explain why plasma concentrations of retinol, which is mainly transported via a specific retinol binding protein, and much less via lipoproteins ${ }^{31}$, did not decrease during exercise. However, others showed that vitamin $\mathrm{E}$ concentrations in muscle tissue also decrease following exercise ${ }^{28}$.

\section{Effect of endurance exercise on LDL oxidation in vitro}

LDL oxidation in vitro was little affected by endurance exercise. The amount of conjugated dienes formed in vitro decreased after exercise by $1.2 \pm 0.6 \%$, and theoretically this indicates that the relative amounts of PUFA in the native LDL had decreased ${ }^{36,37}$. Recently, Sumikawa et al..$^{38}$ showed that exercise decreased the proportion PUFA in RBC phospholipids of untrained subjects. 


\section{Effects of fish oil and vitamin E supplementation}

\section{Effect of supplementation on exercise performance}

Exercise performance as measured by validated exercise tests, was not significantly altered by FO or FE supplementation in the present study. The maximum workload capacity increased slightly during the study, but this was similar in all groups. This small increase, however, was insufficient to improve endurance performance during the time trial. In sedentary healthy female $e^{39}$ and male ${ }^{40}$ subjects, no effects of fish oil supplementation on $\mathrm{W}_{\max }{ }^{39}$ or $\mathrm{VO}_{2}$ max ${ }^{39,40}$ was found either. In well-trained subjects, Leaf and Rauch ${ }^{41}$ found no improvement in treadmill performance after fish oil supplementation. Although they found an increase in $\mathrm{VO}_{2} \max$ (predicted $\mathrm{VO}_{2 \max }$, based on the treadmill performance) after daily supplementation with $6 \mathrm{~g}$ of fish oil, no such increase was noticed after supplementation with $12 \mathrm{~g} /$ day. Analysis of covariance between pre- and post-supplementation predicted $\mathrm{VO}_{2 \max }$ values, however, showed no significant differences between the placebo, low $(6 \mathrm{~g} / \mathrm{d})$, and high (12 $\mathrm{g} / \mathrm{d})$ fish oil group. Therefore, results from this study are difficult to interpret.

\section{Effect of supplementation on RBC characteristics}

The approximately $2 \%$ increase in n-3 PUFA in membrane phospholipids of RBC after fish oil supplementation did not affect RBC deformability. Results from other studies investigating changes in RBC deformability after fish oil supplementation are conflicting. Earlier studies showed that, in young subjects, fish oil supplementation increased whole blood, and $\mathrm{RBC}^{2}$ filterability, but no control groups were used. Recently, Kobayashi et $a l^{42}$ also found an increase in whole blood filterability after fish oil supplementation in healthy young subjects, but not in healthy middle-aged subjects. Guezennec et al. ${ }^{25}$ reported that fish oil supplementation in young trained subjects, as compared with non-supplemented young trained controls, improved filterability of washed RBC after hypoxic exercise. Pre-exercise RBC filterability, however, was not improved by fish oil supplementation ${ }^{25}$. A dose-response study also showed no effect of supplementation with up to $6 \mathrm{~g} /$ day of $\mathrm{n}-3$ ethyl ester fatty acids on filterability of $10 \%$ RBC solutions ${ }^{43}$.

Results were not in agreement even when the proportion of $n-3$ fatty acids in erythrocyte phospholipids increased similarly (the present study vs Refs 1 and 42; Ref 2 vs Ref 25). However, since not all studies were specifically designed to investigate 
the effect of fish oil per se, some of them lack a control group and data should be viewed with caution.

Although decreases in RBC deformability during exercise appeared to be prevented by vitamin $E$, this was not statistically significant. However, a protective effect of vitamin $E$ supplementation on RBC filterability was previously shown during a high altitude ascent ${ }^{44}$.

The average osmotic fragility of RBC was not significantly altered by fish oil supplementation, similar to results from Miller et al. ${ }^{45}$. In contrast, Hagve et al. ${ }^{5}$ reported a decrease in osmotic fragility after 2 weeks of fish oil supplementation, but after 4 weeks of supplementation the fragility approached pre-supplementation values again. They suggested that the initial decrease due to increased unsaturation of membrane fatty acids, was followed by adaption, mediated by a change in phospholipid sub-class distribution.

\section{Effect of supplementation on plasma antioxidants}

FO supplementation did not affect plasma antioxidant concentrations. FO and FE supplementation limited the loss of plasma antioxidants during exercise, but this was not statistically significant. A decrease in plasma vitamin $E$ concentrations after fish oil supplementation has been reported ${ }^{32}$, but this was not confirmed in the present study, or by others ${ }^{46}$. Also, no significant increase in plasma retinol and B-carotene concentrations, as reported by Nair et al. ${ }^{32}$, were noticed. Additional vitamin $\mathrm{E}$ supplementation, however, increased plasma $\alpha$-tocopherol and decreased plasma $\beta+\gamma$-tocopherol concentrations, as has been found by others ${ }^{28}$.

\section{Effect of supplementation on LDL oxidation in vitro}

The lagtime during oxidation of LDL in vitro was not affected by fish oil supplementation, and was only non-significantly increased by additional vitamin $E$ supplementation. A previous study in sedentary male subjects ${ }^{7}$ showed a larger increase in lagtime after FE supplementation as compared with subjects receiving FO supplementation, who showed a decrease in lagtime. Recently, Suzukawa et al. ${ }^{47}$ also found a decrease in lagtime after fish oil as compared with com oil supplementation in drug-treated hypertensive subjects. A dose-response study by Jialal $\theta t a l .^{48}$, showed prolonged lagtimes in subjects supplemented with vitamin $E$, but changes were only significant after $400 \mathrm{IU} /$ day or more, and not after 
supplementation up to $200 \mathrm{IU} /$ day. Lagtimes of EDTA-containing LDL, oxidized with high copper concentrations, were already prolonged after supplementation with 25 to $50 \mathrm{IU} / \mathrm{day}^{49}$.

In the present study, both $\mathrm{FO}$ and FE supplementation reduced the rate of LDL oxidation in vitro. This was not found in a previous study ${ }^{7}$, but recently also reported by Suzukawa et al. ${ }^{47}$. On the other hand, n-6 PUFA increase the rate of oxidation ${ }^{36}$. Kleinveld et al ${ }^{37}$ reported that the rate of oxidation of LDL in vitro, correlated positively with its n-6 PUFA content and negatively with its monoene content. Correlations with $\mathrm{n}-3$ fatty acids, however, were not investigated. Fish oil supplementation in the present study also decreased the ratio of n-6 PUFA to monoenes in RBC phospholipids, and the decrease in rate of oxidation may therefore be related to a similar change in LDL fatty acids.

The decrease in rate of oxidation in the FO group hardly affected $T R_{m}$. Additional vitamin E supplementation, however, delayed oxidation of LDL, although the apparent difference with the FO group was only of borderline significance.

Much to our surprise fish oil supplementation did not affect the amount of conjugated dienes formed during LDL oxidation in vitro, whereas a previous study ${ }^{7}$ showed a $20 \%$ increase after supplementation with a similar amount of fish oil. In addition, the in vitro formation in LDL samples of thiobarituric acid reactive substances after fish oil supplementation ${ }^{46,47}$, and of conjugated dienes after an n-6 PUFA rich diet as compared with a monoene rich diet $^{36}$, were also increased. Although the fast method of LDL preparation used in the present study was different from the time-consuming method in the previous study ${ }^{7}$, comparison of the two methods in six not supplemented sedentary volunteers showed no significant difference in the amount of conjugated dienes formed in vitro (results not reported).

In conclusion, this study showed that exercise decreases RBC deformability, causes consumption or a shift of plasma tocopherols and carotenoids, and decreases the formation of conjugated dienes during oxidation of LDL in vitro. These changes support the theory that oxidative stress during exercise ${ }^{8}$ may decrease RBC deformability (for reviews, see Refs 4 and 9 ). Fish oil supplementation, with or without vitamin $\mathrm{E}$, does not improve RBC deformability or physical performance, but it also does not compromise the plasma antioxidant status. Contrary to previous findings, which indicated that fish oil supplementation increases LDL oxidation, this was not 
found in the present study. Moreover, fish oil supplementation decreased the rate of oxidation in vitro, and additional vitamin $E$ supplementation also delayed the time at which this maximum was reached by a combined effect with a prolonged lagtime.

\section{Acknowledgment}

This study was supported by a grant of the Isostar Sport Nutrition Foundation.

\section{REFERENCES}

1. Terano $T$, Hirai A, Hamazaki $T$, Kobayashi $S$, Fujita $T$, Tamura $Y$, Kumagai A. Effect of oral administration of highly purified eicosapentaenoic acid on platelet function, blood viscosity and red cell deformability in healthy human subjects. Atherosclerosis 1983; 46:321-331.

2. Cartwright IJ, Pockley AG, Galloway JH, Greaves M, Preston FE. The effects of dietary w-3 polyunsaturated fatty acids on erythrocyte membrane phospholipids, erythrocyte deformability and blood viscosity in healthy volunteers. Atherosclerosis 1985; 55:267-281.

3. Bruckner G, Webb P, Greenwell L, Chow C, Richardson D. Fish oil increases peripheral capillary blood cell velocity in humans. Atherosclerosis 1987; 66:237-245.

4. Szygula Z. Erythrocytic system under the influence of physical exercise and training. Sports Med 1990; 10:181-197.

5. Hagve TA, Lie $\emptyset$, Grønn $M$. The effect of dietary $n-3$ fatty acids on osmotic fragility and membrane fluidity of human erythrocytes. Scand J Clin Lab Invest 1993; 53(suppl 215):75-84.

6. Halliwell B, Gutteridge JMC. The importance of free radicals and catalytic metal ions in human diseases. Mol Aspects Med 1985; 8:89-193.

7. Oostenbrug GS, Mensink RP, Homstra G. Effects of fish oil and vitamin E supplementation on copper-catalysed oxidation of human low density lipoprotein in vitro. Eur J Clin Nutr 1994; 48:895-898.

8. Jenkins RR. Free radical chemistry. Relation to exercise. Sports Med 1988; 5:156-170.

9. Smith JA. Exercise, training and red blood cell tumover. Sports Med 1995; 19:9-31.

10. Reinhart $W H$, Stäubli M, Straub PW. Impaired red cell filterability with elimination of old red blood cells during a 100-km race. J Appl Physiol 1983; 54:827-830.

11. Galea G, Davidson RJL. Hemormeology of marathon running. Int J Sports Med 1985; 6:136-138.

12. van der Brug GE, Peters HPF, Hardeman MR, Schep G, Mosterd WL. Hemorheological response to prolonged exercise; no effects of different kinds of feedings. Int J Sports Med 1995; 16:231-237.

13. Pfafferott $C$, Meiselman $H J$, Hochstein P. The effect of malonyldialdehyde on erythrocyte deformability. Blood 1982; 59:12-15.

14. Keizer HA, Kuipers $H$, van Kranenburg G, Geurten P. Influence of liquid and solid meals on muscle glycogen resynthesis, plasma fuel hormone response, and maximal physical working capacity. Int J Sports Med 1986; 8:99-104.

15. Jeukendrup A, Saris WHM, Brouns F, Kester ADM. A new validated endurance performance test. Med Sci Sports Exerc 1996; 28:266-270. 
16. Dill DB, Costill DL. Calculation of percentage changes in volumes of blood, plasma, and red cells in dehydration. J Appl Physiol 1974; 37:247-248.

17. Hardeman MR, Goedhart PT, Dobbe JGG, Lettinga KP. Laser-assisted optical rotational cell analyser (L.O.R.C.A.): I. A new instrument for measurement of various structural hemorheological parameters. Clin Hemorheol 1994; 14:605-618.

18. Hardeman MR, Goedhart PT, Schut NH. Laser-assisted optical rotational cell analyser (L.O.R.C.A.); II Red blood cell deformability: elongation index versus cell transit time. Clin Hemorheol 1994; 14:619-630.

19. Foreman-van Drongelen MMHP, van Houwelingen AC, Kester ADM, Blanco CE, Hasaart THM, Homstra $G$. Influence of feeding artificial formulas containing docosahexaenoic and arachidonic acids on the postnatal long chain polyunsaturated fatty acid status of healthy preterm infants. $\mathrm{Br}$ $J$ Nutr (in press).

20. Hess D, Keller HE, Oberlin B, Bonfanti R, Schũep W. Simultaneous determination of retinol, tocopherols, carotenes and lycopene in plasma by means of high-performance liquid chromatography on reversed phase. Int J Vitam Nutr Res 1991; 61:232-238.

21. Esterbauer $\mathrm{H}$, Striegl $\mathrm{G}$, Puhl $\mathrm{H}$, Rotheneder $\mathrm{M}$. Continuous monitoring of in vitro oxidation of human low density lipoprotein. Free Rad Res Commun 1989; 6:67-75.

22. Neuhaus D, Behn C, Gaehtgens P. Haemorheology and exercise: intrinsic flow properties of blood in marathon running. Int $\mathrm{J}$ Sports Med 1992; 13:506-511.

23. Vandewalle $\mathrm{H}$, Lacombe $\mathrm{C}$, Lelièvre $\mathrm{JC}$, Poirot $\mathrm{C}$. Blood viscosity after a 1-h submaximal exercise with and without drinking. Int J Sports Med 1988; 9:104-107.

24. Wood SC, Doyle MP, Appenzeller O. Effects of endurance training and long distance running on blood viscosity. Med Sci Sports Exerc 1991; 23:1265-1269.

25. Guezennec $C Y$, Nadaud JF, Satabin $P$, Lafargue $P$. Influence of polyunsaturated fatty acid diel on the hemornheological response to physical exercise in hypoxia. Int J Sports Med 1989; 10:286-291.

26. Stuart J. Erythrocyte rheology. J Clin Pathol 1985; 38:965-977.

27. Smith JA, Kolbuch-Braddon M, Gillam I, Telford RD, Weidemann MJ. Changes in the susceptibility of red blood cells to oxidative and osmotic stress following submaximal exercise. Eur J Appl Physiol 1995; 70:427-436.

28. Meydani M, Evans WJ, Handelman G, Biddle L, Fielding RA, Meydani SN, Bumill J, Fiatarone MA, Blumberg JB, Cannon JG. Protective effect of vitamin E on exercise-induced oxidative damage in young and older adults. Am J Physiol 1993; 264:R992-R998.

29. Duthie GG, Robertson JD, Maughan RJ, Morrice PC. Blood antioxidant status and erythrocyte lipid peroxidation following distance running. Arch Biochem Biophys 1990; 282:78-83.

30. Vigule CA, Frei B, Shigenaga MK, Ames BN, Packer L, Brooks GA. Antioxidant status and indexes of oxidative stress during consecutive days of exercise. J Appl Physiol 1993; 75:566-572.

31. Combs GF. The Vitamins. Fundamental Aspects in Nutrition and Health. San Diego: Academic Press, 1992.

32. Nair PP, Judd JT, Berlin E, Taylor PR, Shami S, Sainz E, Bhagavan HN. Dietary fish oil-induced changes in the distribution of $\alpha$-tocopherol, retinol, and $\beta$-carotene in plasma, red blood cells, and platelets: modulation by vitamin E. Am J Clin Nutr 1993; 58:98-102.

33. Taskinen M-R, Nikkila EA, Rehunen S, Gordin A. Effect of acute vigorous exercise on lipoprotein lipase activity of adipose tissue and skeletal muscle in physically active men. Artery 1980; 6:471-483.

34. Traber MG, Olivecrona T, Kayden HJ. Bovine milk lipoprotein lipase transfers tocopherol to human fibroblasts during triglyceride hydrolysis in vitro. J Clin Invest 1985; 75:1729-1734. 
35. Kayden HJ, Traber MG. Absorption, lipoprotein transport, and regulation of plasma concentrations of vitamin $E$ in humans. J Lipid Res 1993; 34:343-358.

36. Reaven P, Parthasarathy S, Grasse BJ, Miller E, Almazan F, Mattson FH, Khoo JC, Steinberg D, Witztum JL. Feasibility of using an oleate-rich diet to reduce the susceptibility of low-density lipoprotein to oxidative modification in humans. Am J Clin Nutr 1991; 54:701-706.

37. Kleinveld HA, Naber AHJ, Stalenhoef AFH, Demacker PNM. Oxidation resistance, oxidation rate, and extent of oxidation of human low-density lipoprotein depend on the ratio of oleic acid content to linoleic acid content: studies in vitamin E deficient subjects. Free Rad Biol Med 1993; 15:273-280.

38. Sumikawa $K, M u Z$, Inoue T, Okochi T, Yoshida T, Adachi $K$. Changes in erythrocyte membrane phospholipid composition induced by physical training and physical exercise. Eur J Appl Physiol 1993; 67:132-137.

39. Agren JJ, Pekkarinen $\mathrm{H}$, Litmanen $\mathrm{H}$, Hanninen $\mathrm{O}$. Fish diet and physical fitness in relation to membrane and serum lipids, prostanoid metabolism and platelet aggregation in female students. Eur J Appl Physiol 1991; 63:393-398.

40. Brilla LR, Landerholm TE. Effect of fish oil supplementation and exercise on serum lipids and aerobic fitness. J Sports Med Phys Fitness 1990; 30:173-180.

41. Leaf DA, Rauch CR. Omega-3 supplementation and estimated $\mathrm{Vo}_{2}$ max: a double blind randomized controlled trial in athletes. Ann Sport Med 1988; 4:37-40.

42. Kobayashi $S$, Hamazaki $T$, Sawazaki $S$, Nakamura $H$. Reduction in the ADP release from shear-stressed red blood cells by fish oil administration. Thromb Res 1992; 65:353-364.

43. Blonk MC, Bilo HJ, Nauta JJ, Popp-Snijders C, Mulder C, Donker AJ. Dose-response effects of fish-oil supplementation in healthy volunteers. Am J Clin Nutr 1990; 52:120-127.

44. Simon-Schnass I, Korniszewski $L$. The influence of vitamin $E$ on theological parameters in high altitude mountaineers. Int J Vitam Nutr Res 1990; 60:26-34.

45. Miller ME, Anagnostou AA, Ley B, Marshall P, Steiner M. Effect of fish oil concentrates on hemorheological and hemostatic aspects of diabetes mellitus: a preliminary study. Thromb Res 1987; 47:201-214.

46. Harats D, Dabach Y, Hollander G, Ben-Naim M, Schwartz R, Berry EM, Stein O, Stein Y. Fish oil ingestion in smokers and nonsmokers enhances peroxidation of plasma lipoproteins. Atherosclerosis 1991; 90:127-139.

47. Suzukawa M, Abbey M, Howe PR, Nestel PJ. Effects of fish oil fatty acids on low density lipoprotein size, oxidizability, and uptake by macrophages. J Lipid Res 1995; 36:473-484.

48. Jialal I, Fuller $\mathrm{C} J$, Huet BA. The effect of $\alpha$-tocopherol supplementation on LDL oxidation. A dose-response study. Arterioscler Thromb Vasc Biol 1995; 15:190-198.

49. Princen $H M G$, van Duyvenvoorde $W$, Buytenhek $R$, van der Laarse $A$, van Poppel $G$, Gevers Leuven JA, van Hinsbergh VWM. Supplementation with low doses of vitamin $E$ protects LDL from lipid peroxidation in men and women. Arterioscler Thromb Vasc Biol 1995; 15:325-333. 


\title{
Maternal and neonatal plasma lipid-soluble antioxidant levels in normal pregnancy, and the relationship with fatty acid unsaturation
}

\author{
Gerard S. Oostenbrug, Ronald P. Mensink, Monique D.M. Al, Adriana C. v. \\ Houwelingen \& Gerard Homstra
}

\section{ABSTRACT}

During pregnancy, maternal plasma levels of lipids, phospholipids, cholesterol, and lipoproteins increase. Since the amount of the peroxidation-susceptible polyunsaturated fatty acids also increases, antioxidant protection may become compromised during pregnancy. In addition, the high degree of unsaturation of fatty acids in neonatal plasma may affect neonatal antioxidant defences. Therefore, we studied maternal and neonatal plasma levels of various antioxidants during normal pregnancy, and the relation with phospholipid fatty acid levels and fatty acid unsaturation.

From the 1 st to the 3rd trimester, plasma levels of tocopherols $(\delta, \beta+\gamma$, and $\alpha)$ and lutein increased by more than $40 \%(P<0.0001)$, $B$-carotene levels decreased by $19 \%(P<0.0001)$, and levels of other carotenoids remained unchanged. Retinol levels decreased from the 2 nd to the 3rd trimester, but returned to $1 \mathrm{st}$ and 2 nd trimester levels after delivery. After delivery, plasma levels of $\delta$-tocopherol $(P=0.006)$ and $B+\gamma$-tocopherol $(P<0.0001)$ were lower than 3rd trimester levels, whereas postpartum $\alpha$-tocopherol and carotenoid levels were similar. As the phospholipid polyunsaturated fatty acid unsaturation index $\left(\mathrm{Ul}_{\mathrm{abs}}\right)$ also increased during pregnancy, antioxidant levels were adjusted for the change in $\mathrm{UI}_{\text {abs. }}$. However, the $\alpha$-tocopherol $/ \mathrm{l}_{\text {abs }}$ ratio still increased from the 1st to the 3rd trimester. Ratios for several carotenoids and retinol, on the other hand, decreased during pregnancy. After delivery, $B+\gamma$-tocopherol $/ \mathrm{UI}_{\mathrm{abs}}$ ratios were lower than 3 rd trimester levels, whereas retinol/UI $\mathrm{abs}_{\text {ratios }}$ were higher.

Umbilical cord plasma antioxidant levels and antioxidant $/ \mathrm{U}_{\mathrm{abs}}$ ratios, except retinol/UI $\mathrm{abs}_{\mathrm{ab}}$, were lower than maternal values $(P<0.01)$. Significant and consistent cord vs matemal correlations were noticed for plasma levels of $B+\gamma+$ tocopherol and several carotenoids, but not for $\delta$-tocopherol, $\alpha$-tocopherol, lycopene, and retinol.

In conclusion, although during pregnancy tocopherol levels increased concurrently with, or more than, fatty acid unsaturation in phospholipids, the decrease in carotenoid/Ul ${ }_{a b s}$ ratios during gestation, the decrease in $B+\gamma$-tocopherol after delivery, and the low neonatal tocopherol and carotenoid levels may affect lipid peroxidation in vivo, and further research is indicated. 


\section{INTRODUCTION}

Results from a large prospective survey have shown that the total amount of fatty acids in plasma phospholipid increase by $51 \%$ from week 10 to week 40 of gestation?. The largest increases were noticed in the concentrations of saturated $(+57 \%)$ and monounsaturated $(+65 \%)$ fatty acids, but $n-6(+44 \%)$ and $n-3(+41 \%)$ polyunsaturated fatty acids levels were also significantly increased. Since polyunsaturated fatty acids are very susceptible to oxidation, the antioxidant status may become compromised during pregnancy.

Several studies, however, have shown that plasma levels of the lipid-soluble antioxidant vitamin $E$ also increase during pregnancy ${ }^{2-6}$. Increases in plasma vitamin $E$ levels during pregnancy coincide with increased amounts of various plasma lipid classes $^{3}$, and plasma vitamin $E$ levels standardized for plasma total lipid ${ }^{3}$, cholesterol ${ }^{4,7}$, or low density lipoprotein ${ }^{2}$ levels appear to remain constant during pregnancy. However, declining levels of triglyceride-standardized plasma vitamin $E$ levels during pregnancy have been reported as well ${ }^{7}$. Reports on vitamin A (retinol) and total carotenoid levels during pregnancy are inconclusive $e^{6,8,9}$, and information about separate carotenoids during pregnancy is scarce. In addition, the degree of unsaturation of the plasma lipids has not been taken into account.

At birth, umbilical vein plasma levels of vitamin $E^{2,10-12}$, carotenoids ${ }^{13-15}$ and retinol $^{12-16}$ have been found to be very low as compared with matemal levels, but levels of total lipid, lipoproteins, cholesterol, and triglycerides were low as well ${ }^{2,10-12}$. The previous prospective survey ${ }^{1}$ also found low levels of phospholipid fatty acids in cord plasma, but showed that the relative amounts of highly unsaturated fatty acids, like arachidonic acid and docosahexaenoic acid, were higher in umbilical vein plasma phospholipids as compared with matemal plasma phospholipids.

Therefore, we have investigated plasma levels of tocopherol isomers, several carotenoids, and retinol during the course of pregnancy and postpartum, and have compared these levels with the degree of fatty acid unsaturation. In addition, plasma lipid-soluble antioxidant levels and fatty acid unsaturation were measured in umbilical cord plasma. 


\section{METHODS}

The samples from the large prospective survey ${ }^{1}$ had been stored for 2 to 4 years at $-80^{\circ} \mathrm{C}$. Therefore, a pilot study was carried out first to check whether storage had affected antioxidant concentrations.

\section{Subjects}

Pilot study: Effect of storage on plasma lipid-soluble antioxidant concentrations

Lipid-soluble antioxidant levels were studied in EDTA-anticoagulated plasma samples, which had been stored for 5 years at $-80^{\circ} \mathrm{C}$. Fifteen samples had been obtained from women after 6 to 17 weeks of pregnancy. From 8 of these women, a second sample was available, collected 4 to 13 weeks after the first sample, but before 27 weeks of gestation. These 23 samples were compared with samples recently collected from 11 women during the first two trimesters of pregnancy, to check whether antioxidant concentrations in samples stored for 5 years are still within the normal range for antioxidant levels during early and mid gestation. Before analysis, the recently collected samples had been stored for 5 months at $-80^{\circ} \mathrm{C}$.

Main study: Plasma lipid-soluble antioxidant levels during normal pregnancy

EDTA-anticoagulated plasma samples were obtained from a large study in which 140 pregnant women originally entered ${ }^{1}$. After analysis of the phospholipid fatty acid patterns performed for that study, 38 complete sets of $1 \mathrm{st}, 2 \mathrm{nd}$, and 3rd trimester plasma samples, 33 postpartum samples and 28 concurrent umbilical vein plasma samples were available for antioxidant analysis. The women were of Caucasian race, had singleton pregnancies, were normotensive, had no metabolic, cardiovascular, neurological or renal disorder, and did not develop gestational diabetes. The 1st, 2nd, and 3rd trimester plasma samples were collected at 14, 22, and 32 weeks of gestation, respectively. Postpartum and umbilical vein (cord) plasma samples were taken immediately after delivery ${ }^{1}$. Before antioxidant analysis, plasma samples had been stored at $-80^{\circ} \mathrm{C}$ for 2 to 4 years. 


\section{Plasma antioxidants}

Plasma was analyzed for tocopherol isomers, carotenoids and retinol by HPLC on a Inertsil ODS-2, C-18 reversed phase column (GL Sciences, Tokyo, Japan), with $68 \%$ acetonitrile/22\% tetrahydrofuran $7 \%$ methanol $3 \%$ distilled water $(\mathrm{v} / \mathrm{v} / \mathrm{v} / \mathrm{v})$ as mobile phase, as described by Hess et al. ${ }^{17}$ with slight modifications (Chapter 3 ). Fluorescence detection was used for the tocopherol isomers, $\delta$-tocopherol, $\beta+\gamma$-tocopherol, and $\alpha$-tocopherol, for the carotenoid phytofluene, and for retinol. Simultaneously, the carotenoids, lutein, $\beta$-cryptoxanthin, lycopene, $\alpha$-carotene and $\beta$-carotene, were detected by absorbance. In the main study, all samples from one subject and her neonate were analyzed in the same analytical run. Chromatogram peak areas were calculated with the Gynkosoft Chromatography Data System (Gynkotek, Germany), and calibrated against a mixture of the various standard substances dissolved in ethanol/dioxane/acetonitrile (1:1:3). In postpartum plasma samples, matemal as well as cord, an unknown component was detected with a retention time slightly less than that of $\delta$-tocopherol, and, therefore, partly overlapping the $\delta$-tocopherol peak. As this sometimes did not allow good calculation of peak areas, all $\delta$-tocopherol concentrations in the main study were based on peak heights instead. Since pure phytofluene was not available, quantitative determination of this compound was not possible. On a reversed phase column, B-tocopherol co-elutes with $\gamma$-tocopherol, and they are therefore reported together. The canthaxanthin standard eluted separately from the lutein standard, but in plasma samples canthaxanthin levels were too low to be chromatographically separated from the lutein peak. Therefore, concentrations of lutein reported here may also include small traces of canthaxanthin. In 4 samples from the pilot study, an unknown peak prevented correct quantitative determination of lutein, and lutein values of these samples are not reported. Twelve analyses of the stock mixture of antioxidants in 6 runs showed coefficients of variation ranging from $2.4 \%$ to $3.6 \%$ for the various antioxidants. The mean ( \pm SEM) recovery of the intemal standard retinyl acetate added to plasmas was $101 \pm 0.6 \%$, and the detection limit in plasma samples was $0.003 \mu \mathrm{mol} / \mathrm{l}$ for all antioxidants.

$\alpha$-Tocopherol (all-rac), $\gamma$-tocopherol, B-cryptoxanthin, lycopene, and B-carotene standards were a generous gift from F. Hoffmann-La Roche (Basel, Switzerland). $\delta$-Tocopherol, lutein, $\alpha$-carotene and retinol (all-trans) standards were obtained from Sigma Chemical Company, and canthaxanthin standard from Fluka Chemie (Bornem, Belgium). All other chemicals were purchased from Merck (Darmstadt, Germany). 


\section{Plasma phospholipid fatty acids}

Details of the plasma phospholipid fatty acid analysis have been reported before ${ }^{1}$. Briefly, lipids were extracted from plasma using a modified Folch method. Then, the phospholipids were separated on aminopropyl bonded silica columns, and saponified. The fatty acids were transmethylated with boron trifluoride methanol, and quantified on a non-polar capillary column. Fatty acid unsaturation was estimated by calculating the absolute polyene unsaturation index $\left(\mathrm{UI}_{\mathrm{abs}}\right)$ as the sum of the micromolar concentration of each polyunsaturated fatty acid (polyene) in the phospholipids multiplied with its number of double bonds. The relative degree of unsaturation was estimated by the relative polyene unsaturation index. This index was calculated as the sum of the percentage of each polyene (moles/moles total fatty acids * $100 \%$ ) multiplied with its number of double bonds.

\section{Statistics}

In the pilot study, lipid-soluble antioxidant levels in all plasma samples were plotted together by gestational age, as previous studies have shown that plasma vitamin $\mathrm{E}$ levels increase linearly during gestation ${ }^{2,3,6}$. Trends during gestation were evaluated by computing linear regression coefficients and Pearson correlation coefficients ( $r$ ) between gestational age and antioxidant levels. Samples from the same subject collected at different gestational ages were considered as independent samples. Mean antioxidant levels in plasma samples stored for 5 years and in recently collected samples, were compared by Student's t-tests.

In the main study, the four serial matemal samples collected during the 1st, 2nd and 3rd trimester and postpartum, were compared by repeated measures analysis of variance (ANOVA) using least-squares estimates of marginal means for unbalanced designs. Levels were evaluated as compared with levels in the 1st trimester, and as compared with levels in the previous trimester. As this involved five simultaneous comparisons, the level of significance between moments of sampling was set to $P<0.01$. Postpartum matemal and cord samples were compared by paired Student's $t$-test and Speaman correlation coefficients $\left(R_{s}\right)$. To study whether the neonatal antioxidant status was related to maternal antioxidant levels before delivery, Spearman 
correlation coefficients were also computed between neonatal values and matemal values during each trimester. Spearman correlation coefficients were preferred since in one or two sets of matemal and neonatal plasma samples, antioxidant levels were considerably higher than in the other sets, which largely affected Pearson correlation coefficients. Values are reported as means \pm SEM. All statistical analyses were performed using the Statistical Analysis System (SAS Institute Inc., 1989).

\section{RESULTS}

Pilot study: Effect of storage on plasma lipid-soluble antioxidant concentrations

Most lipid-soluble plasma antioxidant levels in recently collected samples were not significantly different from levels in samples stored for 5 years (Figure 4.1 and 4.2).

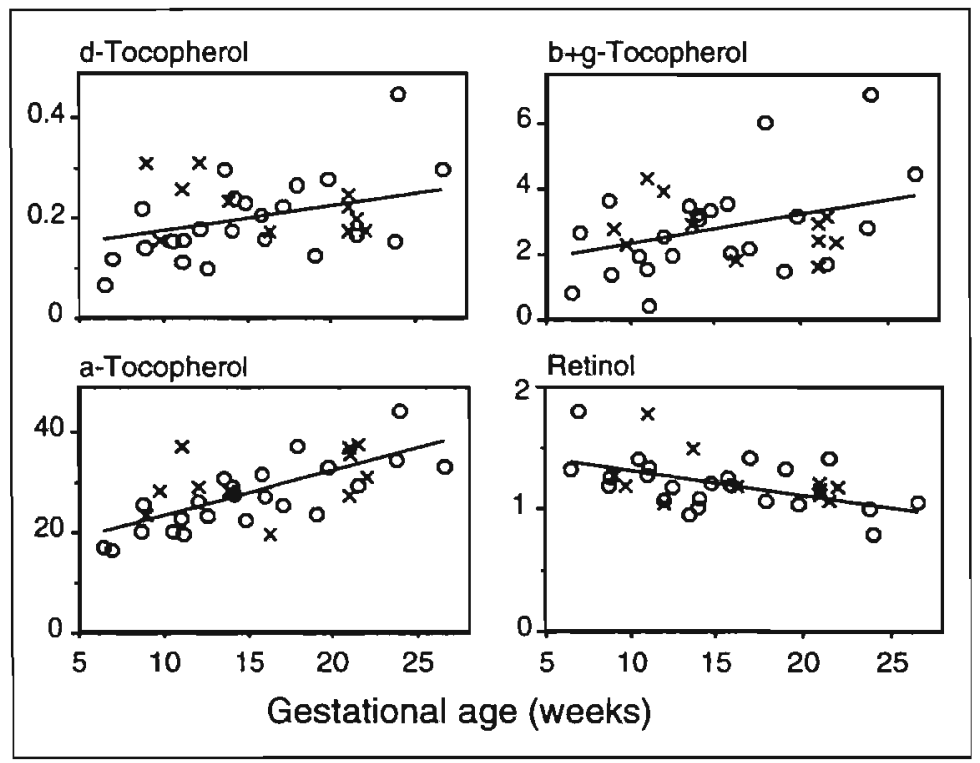

Figure 4.1. Matemal plasma $\delta$-tocopherol, $\beta+\gamma$-tocopherol, $\alpha$-tocopherol, and retinol levels $(\mu \mathrm{mol} / /)$ in samples stored for five years (circles) and in recently collected samples $(X)$. Results are depicted by gestational age, and trends during gestation are shown by solid regression lines, when statistically significant (see text). 
However, mean plasma cryptoxanthin levels in samples stored for 5 years $(0.26 \pm 0.03$ $\mu \mathrm{mol} / \mathrm{l})$ were significantly $(\mathrm{P}=0.035)$ lower than levels in recently collected samples $(0.51 \pm 0.10 \mu \mathrm{mol} / \mathrm{l})$. Consistent with previous reports, plasma levels of tocopherols were higher in samples of later gestational age. Regression coefficients for antioxidant levels between 6 and 27 weeks of gestation, indicated a weekly increase of 0.005 $\mu \mathrm{mol} \delta$-tocopherol $(r=0.359, P=0.034), 0.089 \mu \mathrm{mol} B+\gamma$-tocopherol $(r=0.360, P=0.033)$, $0.900 \mu \mathrm{mol} \alpha$-tocopherol $(r=0.698, P<0.0001)$, and a weekly decrease of $0.020 \mu \mathrm{mol}$ retinol $(r=-0.498, P=0.002)$. Lutein levels also tended to increase by $0.009 \mu \mathrm{mol} /$ week $(r=0.349, P=0.054)$. For plasma levels of the other carotenoids no significant inclining or declining trends during the first 6 months of gestation were noticed (Figure 4.2).

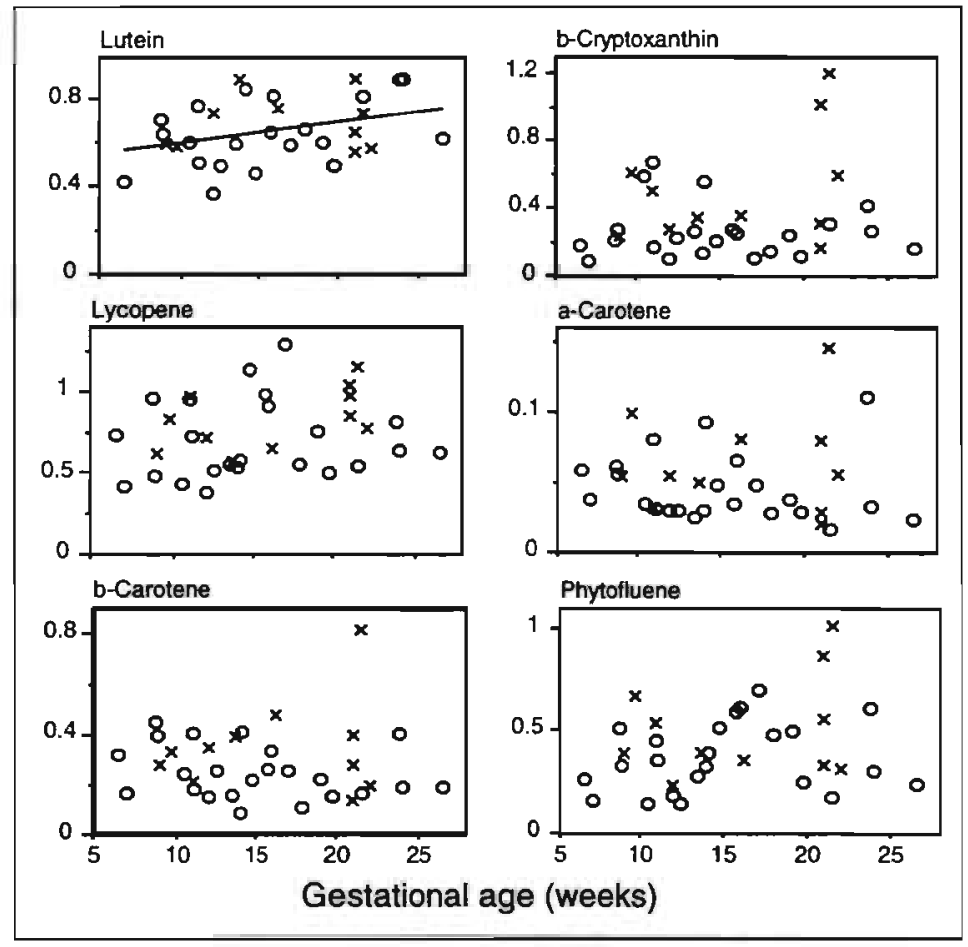

Figure 4.2. Maternal plasma phytofluene, lutein, cryptoxanthin, lycopene, $\alpha$-carotene, and $\beta$-carotene levels in samples stored for five years (circles) and in recently collected samples (X). Antioxidant levels are given in $\mu \mathrm{moV} /$, except for phytofluene levels which is given in arbitrary units. Results are depicted by gestational age, and trends during gestation are shown by solid regression lines, when statistically significant (see text). 


\section{Main study: Plasma lipid-soluble antioxidant levels during normal pregnancy}

All women delivered full term neonates between 37 and 42 weeks of gestation. Birth weights ranged from 2655 to $4310 \mathrm{~kg}$, and of the neonates studied 13 were females and 15 were males.

Plasma levels of tocopherols and lutein increased from the 1st trimester to the 2nd trimester, as was also found in the pilot study, and from the 2nd trimester to the 3rd trimester (Table 4.1). From the 1st to the 3rd trimester, $\delta$-tocopherol levels increased by $74 \%, \beta+\gamma$-tocopherol by $42 \%, \alpha$-tocopherol by $45 \%$, and lutein levels by $42 \%$ $(P<0.0001)$. The intermediate levels in the 2nd trimester were also significantly different from the 1 st trimester for $B+\gamma$-tocopherol $(P=0.006)$ and lutein $(P=0.0006)$, and different from trimester 1 and 3 for $\alpha$-tocopherol $(P<0.0001)$. B-Carotene levels decreased during pregnancy, and levels in the 3rd trimester were $19 \%$ lower than levels in the 1st trimester $(P=0.002)$. Plasma levels of the other carotenoids did not significantly change during pregnancy (Table 4.1). Plasma retinol levels were similar during the 1st and 2nd trimester, but were significantly reduced during the 3rd trimester ( $P<0.0001$ vs other moments of sampling; Table 4.1).

Immediately after delivery, plasma $\alpha$-tocopherol and lutein levels remained increased, but $\delta$-tocopherol $(P=0.006$ vs 3rd trimester) and $B+\gamma$-tocopherol $(P<0.0001$ vs 3rd trimester) levels were lower than levels in the 3rd trimester, and similar to 1st trimester levels. Postpartum plasma levels of other carotenoids were similar to prenatal levels, except for lower lycopene levels as compared with the 1st trimester $(P=0.0004$; Table 4.1). Postpartum retinol levels were similar to levels during the 1 st and 2 nd trimester, and were significantly higher than levels during the 3 rd trimester $(P<0.0001)$.

All fatty acids in the phospholipid fraction increased during pregnancy as well. For saturated and monounsaturated fatty acids, the increase became less strong towards the 3rd trimester. Phospholipid linoleic acid levels, however, increased constantly between each trimester, whereas the increases in n-3 fatty acids appeared to be confined to the first part of gestation. The increase in polyunsaturated fatty acids in phospholipids resulted in an increase in the absolute polyene unsaturation index $\left(\mathrm{UI}_{\mathrm{abs}}\right)$ during pregnancy, but the relative polyene unsaturation index decreased, since the increase in polyenes was less than the increase in other fatty acids (Table 4.2). 
Table 4.1. Maternal plasma antioxidant levels during normal pregnancy, and concurrent neonatal plasma antioxidant levels

\begin{tabular}{|c|c|c|c|c|c|}
\hline $\begin{array}{c}\text { 1st trimester } \\
n=38\end{array}$ & $\begin{array}{l}\text { 2nd trimester } \\
\qquad n=38\end{array}$ & $\begin{array}{c}\text { 3rd trimester } \\
n=38\end{array}$ & $\begin{array}{c}\text { postpartum } \\
n=33\end{array}$ & $\begin{array}{l}\text { cord } \\
n=28\end{array}$ & $\begin{array}{c}\text { C/PP } \\
(\%)\end{array}$ \\
\hline
\end{tabular}

\begin{tabular}{|c|c|c|c|c|c|c|}
\hline $\begin{array}{l}\text { Tocopherols } \\
\delta \text {-tocopherol } \\
\beta+\gamma \text {-tocopherol } \\
\alpha \text {-tocopherol }\end{array}$ & $\begin{aligned} 30.10 & \pm 1.05 \\
0.17 & \pm 0.01 \\
2.70 & \pm 0.16 \\
27.23 & \pm 0.97\end{aligned}$ & $\begin{array}{c}36.62 \pm 1.10^{b} \\
0.21 \pm 0.02 \\
3.20 \pm 0.20^{a} \\
33.20 \pm 1.02^{b}\end{array}$ & $\begin{aligned} 42.59 & \pm 1.29^{b, d} \\
0.24 & \pm 0.02^{b} \\
3.59 & \pm 0.25^{b} \\
38.77 & \pm 1.19^{b, d}\end{aligned}$ & $\begin{array}{r}41.30 \pm 1.78^{b} \\
0.17 \pm 0.01^{c} \\
2.74 \pm 0.19^{d} \\
38.40 \pm 1.73^{b}\end{array}$ & $\begin{array}{l}7.98 \pm 0.274^{e} \\
0.05 \pm 0.007^{e} \\
0.49 \pm 0.043^{e} \\
7.71 \pm 0.346^{e}\end{array}$ & $\begin{array}{l}21 \% \\
30 \% \\
20 \% \\
22 \%\end{array}$ \\
\hline $\begin{array}{l}\text { Carotenoids } \\
\text { lutein } \\
\text { B-cryptoxanthin } \\
\text { lycopene } \\
\alpha \text {-carotene } \\
\text { B-carotene }\end{array}$ & $\begin{array}{l}1.70 \pm 0.09 \\
0.49 \pm 0.03 \\
0.24 \pm 0.03 \\
0.63 \pm 0.04 \\
0.06 \pm 0.01 \\
0.29 \pm 0.02\end{array}$ & $\begin{array}{l}1.74 \pm 0.08 \\
0.59 \pm 0.04 b \\
0.28 \pm 0.03 \\
0.56 \pm 0.03 \\
0.06 \pm 0.00 \\
0.26 \pm 0.02\end{array}$ & $\begin{array}{l}1.78 \pm 0.08 \\
0.67 \pm 0.04 b \\
0.26 \pm 0.02 \\
0.58 \pm 0.04 \\
0.05 \pm 0.00 \\
0.23 \pm 0.02 a\end{array}$ & $\begin{array}{l}1.71 \pm 0.12 \\
0.64 \pm 0.04^{b} \\
0.27 \pm 0.03 \\
0.48 \pm 0.03^{b} \\
0.06 \pm 0.01 \\
0.27 \pm 0.05\end{array}$ & $\begin{array}{l}0.28 \pm 0.029^{\ominus} \\
0.15 \pm 0.017^{\circ} \\
0.04 \pm 0.005^{\theta} \\
0.04 \pm 0.005^{\theta} \\
0.01 \pm 0.001^{\ominus} \\
0.03 \pm 0.004^{\ominus}\end{array}$ & $\begin{array}{r}18 \% \\
26 \% \\
19 \% \\
9 \% \\
19 \% \\
16 \%\end{array}$ \\
\hline phytofluene & $0.62 \pm 0.05$ & $0.59 \pm 0.04$ & $0.68 \pm 0.05$ & $0.61 \pm 0.05$ & $0.00-0.04^{\star}, e$ & \\
\hline Retinol & $1.37 \pm 0.04$ & $1.34 \pm 0.04$ & $1.11 \pm 0.04^{b, d}$ & $1.33 \pm 0.07^{d}$ & $0.67 \pm 0.036^{e}$ & $54 \%$ \\
\hline
\end{tabular}

Plasma collected from pregnant women during each trimester of pregnancy and immediately postpartum, were analyzed for lipid-soluble antioxidant levels. Concurrent umbilical vein plasma samples (cord) were analyzed as well. C/PP expresses the average ratio of cord vs postpartum maternal levels. Values are means \pm SEM. Plasma antioxidant levels are expressed in $\mu$ mol/, except for phytofluene levels which chromatogram peak areas are expressed in $\mathrm{mV}^{*} \mathrm{~min} / \mu \mathrm{l}$ plasma (amplification 100).
$a, b$ : significantly different from 1st trimester:
c. d: significantly different from previous in line:
$a, P<0.01 ; b, P<0.001$ (repeated measures ANOVA).
c, $P<0.01 ; d, P<0.001$ (repeated measures ANOVA).

- significantly different from maternal postpartum levels:

* only 5 cord samples contained detectable concentrations of phytofluene (range is given). 
Table 4.2. Fatty acid composition of matemal plasma phospholipids during normal pregnancy, and neonatal fatty acid composition

$\begin{array}{cccccc}1 \text { st trimester } & \text { 2nd trimester } & \text { 3rd trimester } & \text { postpartum } & \text { cord } & \text { CPP } \\ n=38 & n=38 & n=38 & n=33 & n=28 & (\%)\end{array}$

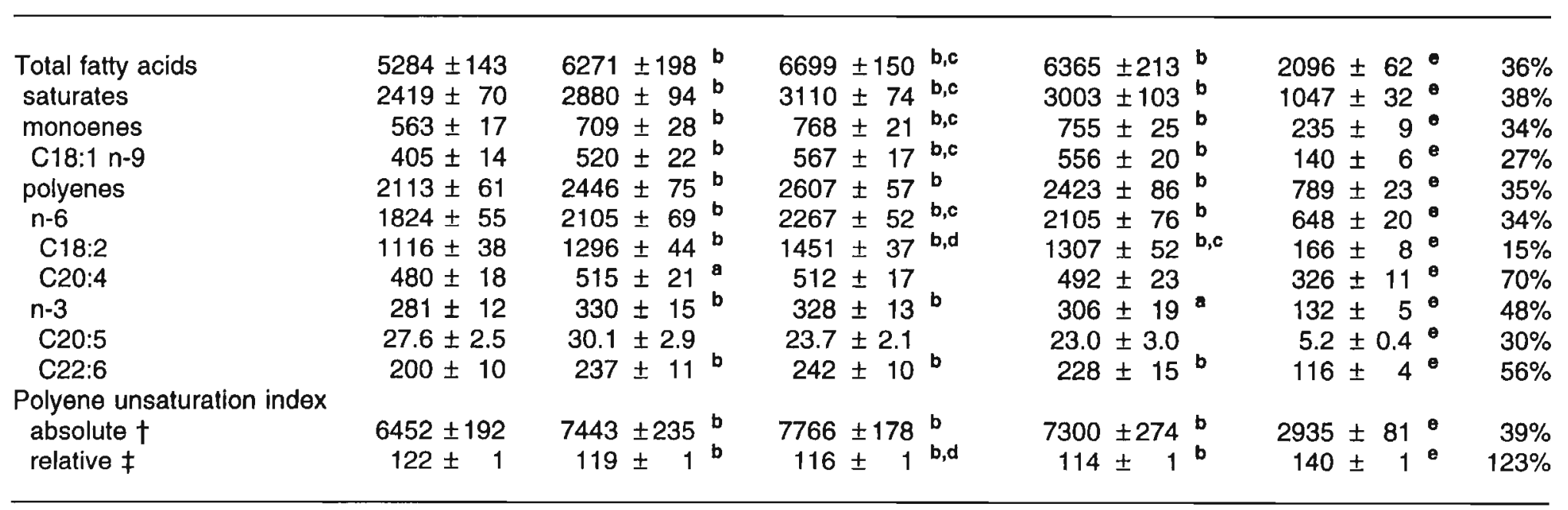

Plasma collected trom pregnant women during each trimester of pregnancy and immediately postpartum, were analyzed for phospholipid fatty acids levels. Concurrent umbilical vein plasma samples (cord) were analyzed as well. C/PP expresses the average ratio of cord vs postpartum maternal levels. Values are means \pm SEM. Plasma phospholipid fatty acid levels are expressed in $\mu$ mol/l. Maternal levels were compared by repeated measures analysis of variance (ANOVA) using least-squares estimates of marginal means for unbalanced designs:

$\begin{array}{ll}\text { a, b: significantly different from 1st trimester: } & \text { a, } P<0.01 ; b, P<0.001 \text { (repeated measures ANOVA). } \\ \text { c, d: significantly different from previous in line: } & \text { c, } P<0.01 ; d, P<0.001 \text { (repeated measures ANOVA). }\end{array}$

- significantly different from maternal postpartum levels: e, $P<0.001$ (Student's paired $t$-test).

+ Calculated as the sum of the micromolar concentration of each polyene multiplied with its number of double bonds.

$¥$ Calculated as the sum of the percentage of each polyene (moles/moles total fatty acids * $100 \%$ ) multiplied with its number of double bonds. 
Table 4.3. Ratio of plasma lipid-soluble antioxidant levels to phospholipid absolute polyunsaturated fatty acid index in normal pregnancy

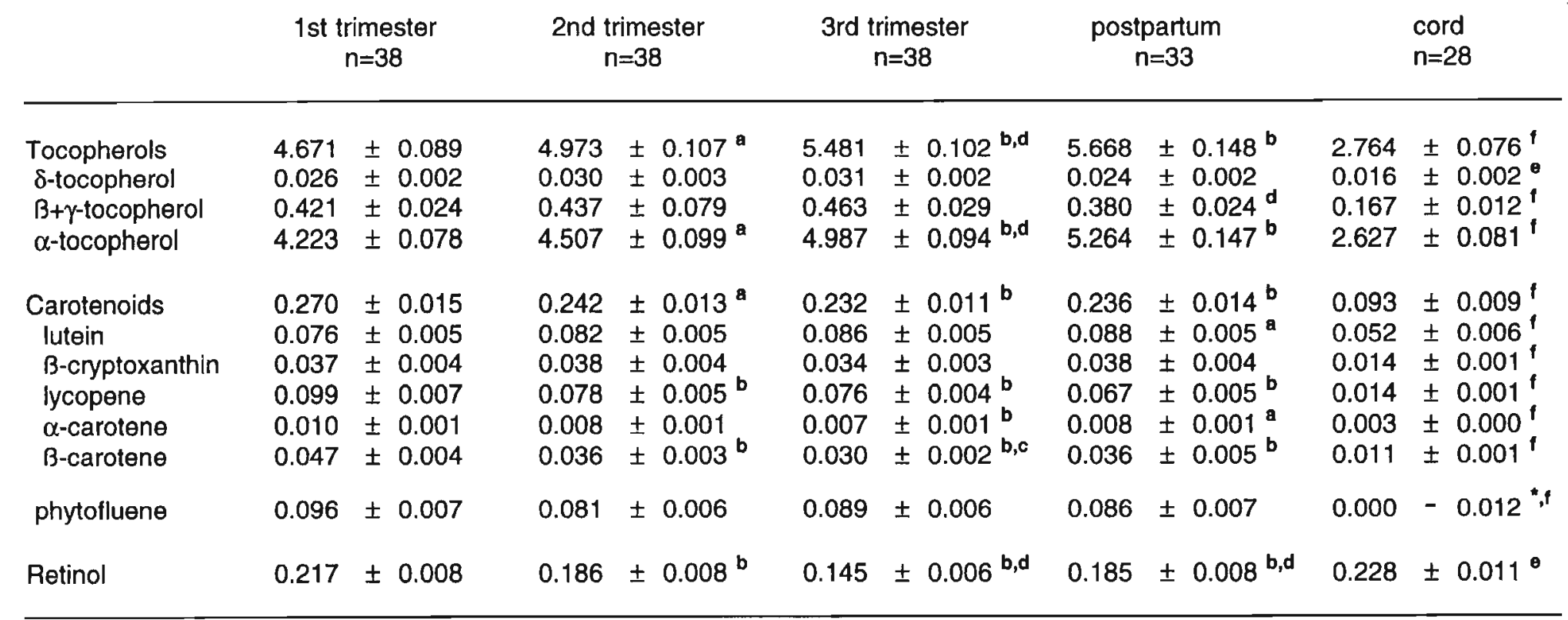

Ratios of plasma lipid-soluble antioxidant to phospholipid absolute polyunsaturated fatty acid index were studied in plasmas from pregnant women during each trimester of pregnancy and immediately postpartum. Ratios in concurrent umbilical vein plasma samples (cord) were calculated as well. Values are means \pm SEM.

a. bignificantly different from 1st trimester:

c. d: significantly different from previous in line:

a, $P<0.01 ; b, P<0.001$ (repeated measures ANOVA).

- significantly different from maternal postpartum levels: $\quad$, $P<0.001$ (Student's paired $t$-test).

${ }^{\star}$, only 5 cord samples contained detectable concentrations of phytofluene (range is given). 
The changes in plasma $\alpha$-tocopherol and lutein levels during pregnancy appeared to coincide with these changes in phospholipid fatty acid levels, but no such relation was evident for the other antioxidants. Nonetheless, plasma $\alpha$-tocopherol to $\mathrm{UI}_{\mathrm{abs}}$ ratios still increased by $20 \%(\mathrm{P}<0.0001)$ during pregnancy (Table 4.3). On the other hand, ratios for lycopene $(-16 \%, P<0.0001), \alpha$-carotene $(-21 \%, P=0.0002), B$-carotene $(-33 \%, \mathrm{P}<0.0001)$ and retinol $(-33 \%, \mathrm{P}<0.0001)$ decreased during pregnancy.

After delivery, $\delta$-tocopherol/UI $I_{\text {abs }}(P=0.023), \alpha$-tocopherol/UI $I_{a b s}(P=0.036)$ and the ratios of the carotenoids/UI $\mathrm{abs}(P>0.017)$ were similar to ratios in the 3rd trimester, but $B+\gamma$-tocophero/ $/ U I_{a b s}$ ratios were significantly decreased $(P=0.0005$; Table 4.3). Postpartum, retinoVUI $\mathrm{abs}_{\text {s }}$ ratios were significantly increased as compared with 3rd trimester ratios $(P<0.0001)$, but were still lower than ratios in the 1 st trimester $(P<0.0001)$.

All cord samples had significantly lower levels of antioxidants than concurrent matemal samples $(P<0.0001)$. Average cord levels of tocopherols and carotenoids were $9 \%$ to $30 \%$ of matemal levels (Table 4.1). Although postpartum $\beta+\gamma$-tocopherol levels in matemal plasma significantly correlated with levels in cord plasma (Table $4.4)$, this was not the case for $\delta$-tocopherol and $\alpha$-tocopherol. Consequently, total tocopherol levels also did not correlate. With the exception of lycopene, carotenoid levels in matemal and cord plasma correlated significantly. In only 5 of 28 cord samples small traces of phytofluene could be detected, and correlation analysis was therefore not performed. Cord retinol levels were on average $54 \%$ of matemal levels, but cord and matemal postpartum levels did not correlate. For $\beta+\gamma-$ tocopherol, lutein, B-cryptoxanthin, and B-carotene neonatal levels also consistently correlated with matemal levels before delivery (Table 4.4). The highest correlation coefficients were found between neonatal and 3rd trimester matemal levels.

Total, saturated, monounsaturated, and n-6 polyunsaturated phospholipid fatty acid levels in cord plasma were on average 32 to $36 \%$ of matemal plasma levels. For $n-3$ fatty acids, the mean cord/matemal ratio was $47 \%$. However, large differences between the various fatty acids were present (Table 4.2). The relative phospholipid polyene unsaturation index was $23 \%$ higher in cord plasma than in matemal plasma, due to a higher proportion of polyunsaturated fatty acids. However, the concentration of total phospholipid fatty acids was lower in cord plasma. Consequently, the absolute polyene unsaturation index was on average $39 \%$ of matemal postpartum values. Nonetheless, ratios of the tocopherols to $\mathrm{UI}_{\mathrm{abs}}$ and carotenoids to $\mathrm{UI}_{\mathrm{abs}}$ were 
significantly lower in cord samples than in postpartum matemal samples (Table 4.3). Retinol/UI $\left.\right|_{a b s}$ ratios, however, were significantly higher than postpartum maternal ratios.

Table 4.4. Spearman correlation coefficients between neonatal and maternal plasma antioxidant levels during pregnancy and postpartum

\begin{tabular}{|c|c|c|c|c|}
\hline \multirow[b]{2}{*}{ Cord antioxidant } & \multicolumn{4}{|c|}{ Maternal levels during gestation } \\
\hline & $\begin{array}{l}\text { 1st } \\
\text { trimester }\end{array}$ & $\begin{array}{c}\text { 2nd } \\
\text { trimester }\end{array}$ & $\begin{array}{c}\text { 3rd } \\
\text { trimester }\end{array}$ & $\begin{array}{c}\text { post- } \\
\text { partum }\end{array}$ \\
\hline Tocopherols & -0.004 & -0.078 & -0.097 & -0.286 \\
\hline $\begin{array}{l}\delta \text {-tocopherol } \\
\text { B+y-tocopherol }\end{array}$ & $\begin{array}{l}0.219 \\
0.592^{b}\end{array}$ & $\begin{array}{l}0.153 \\
0.518^{b}\end{array}$ & $\begin{array}{l}0.407^{a} \\
0.663^{c}\end{array}$ & $\begin{array}{l}0.369 \\
0.585\end{array}$ \\
\hline$\alpha$-tocopherol & 0.079 & 0.011 & -0.069 & -0.152 \\
\hline Carotenoids & $0.430^{a}$ & $0.604^{b}$ & $0.795^{c}$ & $0.461^{8}$ \\
\hline lutein & $0.686^{c}$ & $0.555^{b}$ & $0.812^{c}$ & $0.528^{t}$ \\
\hline B-cryptoxanthin & $0.411^{a}$ & $0.489^{a}$ & $0.764^{c}$ & $0.569^{t}$ \\
\hline lycopene & 0.346 & 0.295 & 0.328 & 0.287 \\
\hline$\alpha$-carotene & 0.274 & 0.141 & 0.381 & $0.515^{\circ}$ \\
\hline B-carotene & $0.500^{a}$ & $0.644^{c}$ & $0.718^{c}$ & $0.510^{2}$ \\
\hline Retinol & -0.054 & -0.132 & 0.128 & 0.183 \\
\hline
\end{tabular}

\section{DISCUSSION}

This study investigated matemal plasma antioxidant levels during pregnancy and its relationship with neonatal antioxidant levels. In addition, antioxidant levels were analyzed in relation to fatty acid unsaturation of plasma phospholipids. A pilot study was performed first to check whether storage of plasma affected lipid-soluble antioxidant concentrations. Only B-cryptoxanthin concentrations appeared to be affected by storage for several years, since concentrations in samples stored for 5 years (pilot study) as well as samples stored for 2-4 years (main study) were considerably lower than concentrations found in recently collected samples (pilot study). Since two recently collected samples contained very high levels of B-cryptoxanthin, comparison between samples stored for 5 years and recently 
collected samples was repeated with a non-parametric Kruskal-Wallis test (not shown). However, the difference between median B-cryptoxanthin levels in the two groups of samples was also statistically significant. Therefore results with respect to this antioxidant should be viewed with caution.

Plasma total tocopherol levels increased significantly during pregnancy, as has been reported for vitamin $E^{2-5}$. Vitamin $E$ is a generic descriptor for tocopherol and tocotrienol isomers, and usually reflects $\alpha$-tocopherol concentrations, the isomer predominantly present. This increase was still present when tocopherol levels were adjusted for the increase in plasma unsaturation as estimated by the phospholipid polyene unsaturation index.

Others have reported constant plasma vitamin $E$ levels during pregnancy, when standardized for total lipid ${ }^{3}$, cholesterol ${ }^{4,7}$, or low density lipoprotein ${ }^{2}$ concentrations, while triglyceride-standardized vitamin $E$ levels decreased during pregnancy ${ }^{7}$. The larger increase in triglycerides, however, may be less important in view of potential oxidative stress, since the degree of unsaturation in triglycerides is relatively low ${ }^{18,19}$. The course of tocopherol levels during pregnancy found in the present study indicates that the increase in phospholipid fatty acids during pregnancy, with emphasis on polyenes, did not compromise the vitamin $E$ status.

Al et al. ${ }^{1}$ suggested that the increase in plasma phospholipid fatty acids levels likely was a consequence of increased mobilization from matemal stores. Plasma vitamin $\mathrm{E}$ levels may have been affected by a similar mechanism, although the increases were larger than the increases in phospholipid fatty acid levels. However, most carotenoid and retinol levels did not increase with phospholipid lipid levels during pregnancy, and the antioxidant/UI $\mathrm{I}_{\text {abs }}$ ratio for these antioxidants decreased. Although this may have affected the antioxidant status, Davidge et al. ${ }^{20}$ reported that the antioxidant capacity of serum to inhibit autoxidation of brain homogenates in vitro, steadily increased during pregnancy. Other studies, in which only total carotenoid levels were measured, have shown an increase ${ }^{6,9}$ or no change ${ }^{8}$ during pregnancy. This discrepancy may be due to the predominance of certain carotenoids in the populations studied, since in our study lutein levels increased and B-carotene levels decreased during pregnancy (Table 4.1). In the present study, retinol levels were only decreased during the 3rd trimester as compared with the 1st and 2nd trimester and postpartum, results comparable to those of Darby et $a l^{6}{ }^{6}$. Morse et al. ${ }^{8}$, however, found no change in plasma vitamin A during pregnancy, whereas Panth et al. ${ }^{9}$ 
reported higher vitamin A levels at mid-gestation, as compared with early and late gestation, in low socio-economic class Indian women. Panth et al. ${ }^{9}$ suggested that the decline during late gestation was due to the poor nutritional status of the women, or increased transfer of retinol across the placenta. In our study, the women were wellnourished, but increased placental transfer may explain the sudden decrease during the 3rd trimester. However, it is unclear why this decrease was then no longer present after delivery.

Several studies have investigated whether the changes in antioxidant levels during pregnancy were sufficient to prevent peroxidation of the increased levels of polyunsaturated fatty acids, but results are conflicting. Although all found an increase in vitamin $\mathrm{E}$ during pregnancy, Wang et al. ${ }^{21}$ found no increase in thiobarbituric acid reactive substances (TBArs), whereas lioka et al. ${ }^{22}$ reported a significant increase in lipid hydroperoxides. Uotila et $a l^{4}$ found increased levels of serum conjugated dienes, only during the 2nd trimester, while serum levels of TBArs decreased nonsignificantly during pregnancy and fluorescent chromolipids did not appear to change.

Postpartum, $\alpha$-tocopherol and carotenoid levels were similar to levels after 32 weeks of gestation. This was previously also found for vitamin $E^{2,5}$, whereas others have reported that the increase in matemal $\alpha$-tocopherol levels during gestation continued until birth ${ }^{3,23}$. Our study, however, showed that $\delta$ - and $B+\gamma$-tocopherol levels were lower immediately after delivery as compared with levels in the 3rd trimester. In a previous study in which oxidative stress was studied in patients undergoing percutaneous transluminal coronary angioplasty, we also noticed a decrease in only $\delta$ - and $B+\gamma$-tocopherol levels in low density lipoproteins 2 days after the procedure, but no decrease in $\alpha$-tocopherol levels ${ }^{24}$. However, in the present study the period between 3rd trimester sampling and partus is too long to substantiate conclusions about possible (oxidative) stress during labor.

Cord plasma of newboms contained lower antioxidant levels than matemal plasma, as has already been reported for vitamin E, B-carotene, total carotenoids, and retinol ${ }^{2,5,9-16,23,25}$. Cord levels were also lower than maternal levels during early gestation, and levels found in men (Chapter 3). Although it has been suggested that the lower vitamin $E$ levels in newborns are due to low total lipid ${ }^{11}$ or low density lipoprotein ${ }^{2}$ levels, in the present study cord total phospholipid fatty acid-standardized tocopherol and carotenoid levels (results not shown) and $\mathrm{UI}_{\text {abs }}$ adjusted ratios were still significantly lower than matemal values. Consistent with our results, Jagadeesan 
et al. ${ }^{10}$ found a lower vitamin E/cholesterol ratio in cord plasma as compared with matemal plasma, whereas the lower neonatal vitamin E/cholesterol ratio in another study ${ }^{12}$ was not statistically significant. Retinol levels in cord plasma were about half of matemal levels, and retinol/LI $\left.\right|_{\text {abs }}$ ratios were significantly higher. It is unknown whether increased placental transfer during late gestation, as suggested by Panth et al. ${ }^{9}$, may explain the relative high cord levels of retinol as compared with the other antioxidants and phospholipid fatty acids.

Lipid-soluble antioxidants act as important radical scavengers in lipophilic compartments, like membranes, but several other compounds may also contribute to antioxidant defences ${ }^{26,27}$. Vitamin C (ascorbic acid) in plasma is an effective scavenger of aqueous peroxyl radicals ${ }^{28}$. Although vitamin $E$ belongs to the first line of antioxidant defence against lipid-soluble peroxyl radicals ${ }^{29}$, vitamin $C$ is capable of regenerating oxidized vitamin $E^{26,27}$, while being consumed. Contrary to lipid-soluble antioxidant levels, however, vitamin $\mathrm{C}$ levels in umbilical vein plasma are higher than levels in maternal plasma ${ }^{30}$. Nonetheless, the total antioxidant capacity of serum to inhibit autoxidation of brain homogenates is lower in neonates than in adults ${ }^{31}$. On the other hand, the total free radical trapping ability of plasma is similar in term neonates and adults ${ }^{32,33}$, and a $\operatorname{low}^{34}$ as well as high $^{35}$ degree of lipid peroxidation has been reported in newborns.

For total tocopherol (vitamin E), cord levels did not correlate with maternal levels, in agreement with other reports ${ }^{10,12}$. Shah et al ${ }^{36}$, however, reported a significant correlation between cord and matemal levels in low-income and high-income Indian subjects. Although matemal levels in this study were only $60 \%$ of levels in the present study, average cord levels were similar or higher. In fact, in groups with high (40-50 $\mu \mathrm{mol} / /$; refs 2,5 , the present study) or low $(23-27 \mu \mathrm{mol} / /$; refs $23,25,36)$ matemal levels at delivery, cord levels appear to be similar (ranging from 8 to 9 , and 6 to 10.5 $\mu \mathrm{mol} / /$, respectively). This may indicate that vitamin $E$ levels in cord blood are only influenced by matemal levels when these maternal levels are low. This is also supported by the higher correlation coefficient found in the low-income group with lower vitamin $E$ levels, as compared with the high-income group ${ }^{36}$. Interestingly, $B+\gamma$-tocopherol levels in cord and matemal plasma were well-correlated. The reason for the differences in neonatal/matemal correlation between the tocopherol isomers remains unclear however.

For several carotenoids, cord plasma levels correlated with matemal levels, and correlation coefficients were highest with 3rd trimester levels. Although this may 
indicate that these carotenoids are concentration-dependently transferred from the mother to the child, this does not appear to be the case for lycopene, since correlation coefficients for this carotenoid were not significant. The low child/mother ratio for lycopene ( $9 \%$ ) as compared with the other carotenoids (16 to $26 \%$ ), also suggests a different transfer or metabolism of lycopene. Furthermore, the apparent absence of phytofluene in cord samples indicates that this carotenoid is not transferred from matemal to cord plasma at all. The absence of phytofluene in cord samples was not due to lack of sensitivity, since we are able to detect phytofluene levels which are 1000 times as low as average matemal levels. This, together with the observed differences in responses of some carotenoids during pregnancy, indicates that measurements of total carotenoid ${ }^{9,14,15}$ or only $B$-carotene ${ }^{13}$ levels may not correctly reflect responses of all carotenoids.

Retinol levels in cord and maternal plasma did not correlate, as has been reported in Scandinavian ${ }^{12}$, US Navajo ${ }^{15}$, and Brazilian ${ }^{16}$ populations. Studies from India ${ }^{37}$ and Egypt ${ }^{14}$, however, reported a significant correlation between cord and maternal levels. Although average matemal retinol levels were lower in the latter studies (0.8$1.1 \mu \mathrm{mol} / \mathrm{l})$ as compared with our study and refs 12,15 , and $16(1.3-1.7 \mu \mathrm{mol} / \mathrm{l})$, Shah et al. ${ }^{37}$ found a higher correlation coefficient in a high-income group with higher retinol levels, as compared with the low-income group.

Although the pregnancies were essentially uncomplicated, two women developed mild pregnancy-induced hypertension. Re-analysis of the data excluding these two women did not significantly affect trends during pregnancy, or matemal/neonatal comparisons. A comprehensive comparison between plasma lipid-soluble antioxidant levels in pregnancies complicated with pregnancy-induced hypertension and levels in uncomplicated pregnancies is described in Chapter 5.

In summary, this study showed that during pregnancy the increase in plasma phospholipid fatty acid levels, and thus in peroxidation-susceptible polyunsaturated fatty acids and unsaturation index, was accompanied by an equal or even larger increase in tocopherol levels, but that carotenoid levels did not increase concurrently. Furthermore, $\beta+\gamma$-tocopherol levels decreased after delivery, and umbilical cord levels of tocopherols and carotenoids were lower than matemal levels, even after correction for the lower fatty acid unsaturation found in neonates. Additional studies are necessary to demonstrate whether these changes in antioxidant status affect lipid peroxidation in vivo. 


\section{REFERENCES}

1. AI MDM, Houwelingen AC v, Kester ADM, Hasaart THM, de Jong AEP, Homstra G. Matemal essential fatty acid patterns during normal pregnancy and their relationship to the neonatal essential fatty acid status. Br J Nutr 1995; 74:55-68.

2. Hágå $P, E k J$, Kran S. Plasma tocopherol levels and vitamin E/B-lipoprotein relationships during pregnancy and in cord blood. Am J Clin Nutr 1982; 36:1200-1204.

3. Mino $M$, Nagamatu $M$. An evaluation of nutritional status of vitamin $E$ in pregnant women with respect to red blood cell tocopherol level. Int J Vitam Nutr Res 1986; 56:149-153.

4. Uotila J, Tuimala R, Aamio T, Pyykkō K, Ahotupa M. Lipid peroxidation products, selenium-dependent glutathione peroxidase and vitamin $\mathrm{E}$ in normal pregnancy. Eur $\mathrm{J}$ Obstet Gynecol Reprod Biol 1991; 42:95-100.

5. von Mandach U, Huch R, Huch A. Matemal and cord serum vitamin E levels in nomal and abnormal pregnancy. Int J Vitam Nutr Res 1993; 63:26-32.

6. Darby WJ, McGanity WJ, Martin MP, Bridgforth E, Densen PM, Kaser MM, Ogle PJ, Newbill JA, Stockell A, Ferguson ME, Touster O, McClellan GS, Williams C, Cannon RO. The Vanderbilt cooperative study of maternal and infant nutrition. IV. Dietary, laboratory and physical findings in 2,129 delivered pregnancies. J Nutr 1953; 51:565-597.

7. Jagadeesan V. Prema K. Plasma tocopherol and lipid levels in pregnancy and oral contraceptive users. Br J Obstet Gynaecol 1980; 87:903-907.

8. Morse EH, Clarke RP, Keyser DE, Merrow SB, Bee DE. Comparison of the nutritional status of pregnant adolescents with adult pregnant women. I. Biochemical findings. Am $\mathrm{J}$ Clin Nutr 1975; 28:1000-1013.

9. Panth $M$, Shatrugna V, Yasodhara $P$, Sivakumar B. Effect of vitamin A supplementation on haemoglobin and vitamin A levels during pregnancy. Br J Nutr 1990; 64:351-358.

10. Jagadeesan V, Prema K. Plasma tocopherol and lipid levels in mother and umbilical cord; influence on birth weight. Br J Obstet Gynaecol 1980; 87:908-910.

11. Martinez FE, Goncalves AL, Jorge SM, Desai ID. Brief clinical and laboratory observations. Vitamin $E$ in placental blood and its interrelationship to maternal and newbom levels of vitamin E. J Pediatr 1981; 99:298-300.

12. Uotila J, Tuimala R, Pyykkō K, Ahotupa M. Pregnancy-induced hypertension is associated with changes in matemal and umbilical blood antioxidants. Gynecol Obstet Invest 1993; 36:153-157.

13. Baker $H$, Thind IS, Frank $O$, DeAngelis B, Caterini $H$, Louria DB. Vitamin levels in low-birth-weight newbom intants and their mothers. Am J Obstet Gynecol 1977; 129:521-524.

14. Hussein L, el Shawarby O, Elnaggar B, Abdelmegid A. Serum vitamin A and carotene concentrations among Egyptian fullterm neonates in relation to matemal status. Int $\mathrm{J}$ Vitam Nutr Res 1988; 58:139-145.

15. Butte NF, Calloway DH. Proteins, vitamin A, carotene, folacin, ferritin and zinc in Navajo matemal and cord blood. Biol Neonate 1982; 41:273-278.

16. Rondo PHC, Abbott R, Rodrigues LC, Tomkins AM. Vitamin A, folate, and iron concentrations in cord and matemal blood of intra-uterine growth retarded and appropriate birth weight babies. Eur J Clin Nutr 1995; 49:391-399.

17. Hess D, Keller HE, Oberlin B, Bonfanti R, Schùep W. Simultaneous determination of retinol, tocopherols, carotenes and lycopene in plasma by means of high-performance liquid chromatography on reversed phase. Int J Vitam Nutr Res 1991; 61:232-238. 
18. van der Schouw YT, AI MD, Homstra G, Bulstra-Ramakers MT, Huisjes HJ. Fatty acid composition of serum lipids of mothers and their babies after nomal and hypertensive pregnancies. Prostaglandins Leukot Essent Fatty Acids 1991; 44:247-252.

19. Lorentzen B, Drevon CA, Endresen MJ, Henriksen T. Fatty acid pattern of esterified and free fatty acids in sera of women with normal and pre-eclamptic pregnancy. Br J Obstet Gynaecol 1995; 102:530-537.

20. Davidge ST, Hubel CA, Brayden RD, Capeless EC, McLaughlin MK. Sera antioxidant activity in uncomplicated and preeclamptic pregnancies. Obstet Gynecol 1992; 79:897-901.

21. Wang YP, Walsh SW, Guo J, Zhang J. Maternal levels of prostacyclin, thromboxane, vitamin $E$, and lipid peroxides throughout normal pregnancy. Am J Obstet Gynecol 1991; 165:1690-1694.

22. lioka $H$, Akada $S$, Hisanaga $H$, Shimamoto $T$, Yamada $Y$, Moriyama IS, Ichijo $M$. Changes in plasma levels of lipid peroxide and vitamin $E$ during pregnancy. Asia-Oceania $\mathrm{J}$ Obstet Gynaecol 1991; 17:357-361.

23. Vobecky JS, Vobecky J, Shapcott D, Blanchard R, Lafond R, Cloutier D, Munan L. Serum alpha-tocopherol in pregnancies with normal or pathological outcomes. Can J Physiol Pharmacol 1974; 52:384-388.

24. Oostenbrug GS, Mensink RP, Bär FWHM, Hornstra G. Lipid peroxidation-associated oxidative stress during percutaneous transluminal coronary angioplasty in humans. Free Rad Biol Med (in press).

25. Navarro J, Causse MB, Desquilbet N, Herve F, Lallemand D. The vitamin status of low birth weight infants and their mothers. J Pediatr Gastroenterol Nutr 1984; 3:744-748.

26. Niki E. Antioxidants in relation to lipid peroxidation. Chem Phys Lipids 1987; 44:227-253.

27. Stocker R, Frei B. Endogenous antioxidant defences in human blood plasma. In: Sies $\mathrm{H}$ (ed.). Oxidative Stress. Oxidants and Antioxidants. London: Academic Press, 1991:213-243.

28. Frei B. England L, Ames BN. Ascorbate is an outstanding antioxidant in human blood plasma. Proc Natl Acad Sci USA 1989; 86:6377-6381.

29. Frei B, Stocker R, Ames BN. Small molecule antioxidant defences in human extracellular fluids. In: Scandalios JG (ed.). Molecular Biology of Free Radical Scavenging Systems. Cold Spring Harbor Laboratory Press: New York, 1992:23-45.

30. Guajardo L, Beharry KDA, Modanlou HD, Aranda JV. Ascorbic acid concentrations in umbilical cord veins and arteries of preterm and term newborns. Biol Neonate 1995; 68:1-9.

31. Sullivan JL, Newton RB. Serum antioxidant activity in neonates. Arch Dis Child 1988;63:748757.

32. Lindeman JHN, van Zoeren-Grobben D, Schrijver J, Speek AJ, Poorthuis BJHM, Berger HM. The total free radical trapping ability of cord blood plasma in preterm and term babies. Pediatr Res 1989; 26:20-24.

33. Gopinathan V, Miller NJ, Milner AD, Rice-Evans CA. Bilirubin and ascorbate antioxidant activity in neonatal plasma. FEBS Lett 1994; 349:197-200.

34. Deligné P, Bonnardot JP, Couderc R, Kerisit S, Périer JF, Laruelle P. Lipid peroxidation levels and antioxidant activities of blood plasma in parturients and new-bom infants immediatly after normal delivery. Adv Exp Med Biol 1990; 264:573-576.

35. Takehara $Y$, Yoshioka T, Sasaki J. Changes in the levels of lipoperoxide and antioxidant factors in human placenta during gestation. Acta Med Okayama 1990; 44:103-111.

36. Shah RS, Rajalakshmi R, Bhatt RV, Hazra MN, Patel BC, Swamy NB, Patel TV. Vitamin E status of the newborn in relation to gestational age, birth weight and matemal vitamin $E$ status. Br J Nutr 1987; 58:191-198. 



\section{Pregnancy complicated with pregnancy-induced hypertension: maternal and neonatal plasma lipid-soluble antioxidant levels and its relationship with fatty acid unsaturation}

Gerard S. Oostenbrug, Ronald P. Mensink, Monique D.M. Al, Adriana C. v. Houwelingen \& Gerard Homstra

\section{ABSTRACT}

It has been suggested that the plasma antioxidant status may be decreased in women with pregnancy-induced hypertension (PIH). However, results are inconclusive, and only a limited number of antioxidants have been studied. Therefore, we have compared plasma levels of vitamin $E$ isomers, several carotenoids, and vitamin $A$ during the 3rd trimester and postpartum in 23 women with PIH and their neonates, with levels in 23 matched controls with uncomplicated pregnancies. Most (19) women with PIH, however, had diastolic blood pressures under $110 \mathrm{~mm} \mathrm{Hg}$ throughout pregnancy, and can be classified as mild cases.

In the 3rd trimester of pregnancy, lipid-soluble antioxidant levels were similar in women with $\mathrm{PIH}$ and controls. In the control group, $B+\gamma$-tocopherol levels decreased on average by $5 \%(P=0.038)$ from the 3rd trimester to postpartum. In the PIH group, however, plasma levels of $\delta$-tocopherol, $\beta+\gamma$-tocopherol, lutein, $\alpha$-carotene, and $\beta$-carotene decreased by $15 \%$ to $26 \%$ $(P<0.02)$. As compared with changes in the control group, $B+\gamma$-tocopherol, lutein, and $\alpha$-tocopherol levels in the PIH group decreased significantly $(P<0.03)$. At the same time, the absolute phospholipid polyunsaturated fatty acid unsaturation index $\left(\mathrm{UI}_{\mathrm{abs}}\right.$ ) decreased in the $\mathrm{PIH}$ group, as compared with the control group.

The ratios of $B+\gamma$ tocopherol and lutein to $\mathrm{Ul}_{\text {abs }}$ decreased in the control group from the 3rd trimester to postpartum, but ratios for several other antioxidants decreased in the PIH group as well. As compared with controls, however, these decreases did not reach statistical significance. Umbilical vein plasma antioxidant levels were similar after complicated and uncomplicated pregnancies.

In conclusion, these findings do not support the hypothesis that plasma lipid-soluble antioxidant levels in the mother and the child are affected by mild PIH. However, this study does not exclude the possibility that antioxidant levels may be more affected in women with severe PIH. 


\section{INTRODUCTION}

Recently, it has been suggested that many of the clinical and pathophysiological features of pregnancy-induced hypertension $(\mathrm{PIH})$ might be explained by an imbalance between lipid peroxidation and antioxidant status (review by $\mathrm{Walsh}^{1}$ ). Especially the role of the lipid-soluble antioxidants, like vitamin $E$, is emphasized ${ }^{1}$. They stabilize biological membranes and protect them from oxidative damage. Indeed, during the 3rd trimester of pregnancy, the antioxidant capacity of serum to inhibit autoxidation of brain homogenates ${ }^{2}$, and plasma vitamin $E$ levels ${ }^{3}$, were significantly lower in women with severe $\mathrm{PIH}$ as compared with uncomplicated pregnancies. However, Uotila et al. ${ }^{4}$ reported increased rather than decreased vitamin $E$ levels during severe $\mathrm{PIH}$. In women with mild PIH, plasma vitamin E levels during the 3rd trimester were not increased ${ }^{3,4}$. On the other hand, the total peroxyl radical-trapping capacity (TRAP) of plasma shortly before delivery was higher in women with mild or severe $\mathrm{PIH}^{5}$. Therefore, these studies are inconclusive with respect to the plasma antioxidant status in PIH.

After delivery, plasma vitamin $\mathrm{E}$ levels were similar in women with mild or severe $\mathrm{PIH}$, as compared with uncomplicated pregnancies ${ }^{6}$. The antioxidant capacity of serum to inhibit autoxidation of brain homogenates, however, was decreased in women with severe $\mathrm{PIH}^{2}$. Umbilical cord plasma and serum vitamin $E$ levels were atso similar after complicated and uncomplicated pregnancies ${ }^{6.7}$. However, cord plasma vitamin A levels were lower after pregnancies complicated with $\mathrm{PIH}^{6}$.

Reports on other lipid-soluble antioxidants during $\mathrm{PIH}$ are scarce and also inconclusive ${ }^{6,8,9}$. In addition, we have previously shown that plasma levels of the separate isomers of vitamin $E$ and various carotenoids, do not change uniformly during pregnancy (Chapter 4), and further analysis of these isomers is indicated. Therefore, we have studied levels of three vitamin E isomers, several carotenoids, and vitamin A in women with PIH and their neonates. In addition, we have compared these levels with the degree of fatty acid unsaturation of phospholipids. There is considerable evidence that endothelial cell injury is involved in the pathogenesis of $\mathrm{PIH}^{10}$, and phospholipids form important components of cell membranes. Furthermore, the high degree of fatty acids with three or more double bonds in phospholipids ${ }^{11}$ makes them highly susceptible to peroxidation, and fatty acid peroxidation may have consequences for membrane integrity ${ }^{12}$. 


\section{METHODS}

\section{Study population}

This prospective study was initially set up to examine the essential fatty acid status of mother and child ${ }^{13}$. Informed consent was received from each participant, and prior approval for the study was obtained from the Medical Ethics Committee of the University of Limburg. The cohort consisted of pregnant women with a diastolic pressure (DBP) lower than $90 \mathrm{~mm}$ $\mathrm{Hg}$, and who did not suffer from cardiovascular, neurological, renal or metabolic disorders at entry for the study, which was before week 16 of gestation. Only women who intended to deliver at the hospital and had singleton pregnancies were included. After delivery, medical records were checked, and women with a DBP of $90 \mathrm{~mm} \mathrm{Hg}$ or more on two consecutive measurements with an interval of at least of 4 hours, one measurement of $110 \mathrm{~mm} \mathrm{Hg}$ or more after 20 weeks of gestation, or an increase of at least $25 \mathrm{~mm} \mathrm{Hg}$ during pregnancy, were classified as $\mathrm{PIH}^{14}$. These women were matched for parity and hospital with three women with uncomplicated pregnancies (controls). Venous plasma samples were collected during the 1st, 2nd, 3rd trimester, and immediately after delivery. Postpartum, umbilical cord (vein) plasma was collected as well.

After phospholipid tatty acid analysis for that study, 23 sets of samples from women with $\mathrm{PIH}$ and their concurrent neonates were available for antioxidant analysis. Only maternal samples from the 3rd trimester (approximately 32 weeks of gestation) and maternal and neonatal postpartum samples were analyzed, because of the reported differences between $\mathrm{PIH}$ and uncomplicated pregnancies with regard to plasma antioxidant levels and plasma antioxidant capacities during this period of pregnancy (see introduction). For each PIH patient, 1 matched control was randomly selected from the 3 available controls per patient. Until analysis, plasma samples had been stored for 2 to 5 years at $-80^{\circ} \mathrm{C}$. For further details see ref. 13. 


\section{Plasma antioxidants}

EDTA-anticoagulated plasma was analyzed by HPLC for the vitamin E isomers, $\delta$-tocopherol, $\beta+\gamma$-tocopherol and $\alpha$-tocopherol, for the carotenoids, lutein, $\beta$-cryptoxanthin, lycopene, $\alpha$-carotene and B-carotene, and for retinol (vitamin A), as described in Chapter 4. All $\delta$-tocopherol concentrations were based on chromatogram peak heights, as an unknown peak in postpartum samples sometimes prevented good calculation of peak areas. Since pure phytofluene was not available, quantitative determination of this compound was not possible (for further details, see Chapter 4). All samples from one subject, her neonate, and the samples from the matched control were analyzed in the same analytical run. Storage of plasma at $-80^{\circ} \mathrm{C}$ for 5 years was shown not to affect lipid-soluble antioxidant concentrations (Chapter 4). Between-run coefficients of variation for a mixture of standards ranged from $1.1 \%$ to $2.2 \%$ for the various antioxidants. The mean ( \pm SEM) recovery of the internal standard retinyl acetate added to plasmas was $97 \pm 0.5 \%$, and the detection limit in plasma samples was $0.003 \mu \mathrm{mo} / / \mathrm{l}$ for all antioxidants.

\section{Plasma phospholipid fatty acids}

Details of the plasma phospholipid fatty acid analysis have been reported before ${ }^{13}$. Briefly, lipids were extracted from plasma using a modified Folch method. Then, the phospholipids, separated on aminopropyl bonded silica columns, were saponified, and their fatty acids were transmethylated with boron trifluoride methanol. Fatty acids were separated by gas-liquid chromatography on a non-polar capillary column, and identified by flame ionization detection and authentic standards ${ }^{13}$. To quantify peak areas, a standard solution of $1-\alpha$-dinonadecanoyl lecithin was added to each plasma sample before extraction. The absolute phospholipid polyene unsaturation index ( $\left.\mathrm{Ul}_{\mathrm{abs}}\right)$ was calculated as the sum of the micromolar concentration of each polyunsaturated fatty acid in the phospholipids multiplied with its number of double bonds. The relative polyene unsaturation index was calculated as the sum of the percentage of each polyene (moles/moles total fatty acids * $100 \%$ ) multiplied with its number of double bonds. 


\section{Statistics}

Paired Student's $t$-tests were used to compare clinical parameters and 3rd trimester plasma values in the $\mathrm{PIH}$ group with those in the control group. Since the changes in phospholipid polyunsaturated fatty acids from the 3rd trimester to postpartum were significantly different between PIH and uncomplicated pregnancies ${ }^{13}$, paired $t$-tests were also used to evaluate the changes from 3rd trimester to postpartum within each group, and between the two groups. Furthermore, calculation of these changes made it possible to correct data for intrinsic differences. Paired $t$-statistics were also computed to compare umbilical cord plasma levels in the PIH group with those in the control group, and to compare cord levels with maternal postpartum levels. Pearson correlation coefficients were calculated between DBP and antioxidant levels for all subjects grouped together, and between changes in these parameters from the 3rd trimester to postpartum. The level of significance was set to $P<0.05$. Values are reported as means $\pm S E M$. All statistical analyses were performed using the Statistical Analysis System (SAS Institute Inc., 1989).

\section{RESULTS}

In the control group, aged $29.2 \pm 0.9$ years, 7 smoked, and their mean DBP at entry was $67.0 \pm 1.6 \mathrm{~mm} \mathrm{Hg}$. In the $\mathrm{PIH}$ group, aged $28.0 \pm 0.6$ years $(P=0.257$ vs controls), 3 smoked, and their DBP at entry was $72.0 \pm 1.7 \mathrm{~mm} \mathrm{Hg}$ ( $P=0.026$ vs controls). In both groups, 19 women were nulliparae at entry, and 4 women delivered their second baby. During pregnancy, the mean maximum DBP in the control group was $75.3 \pm 0.8 \mathrm{~mm} \mathrm{Hg}$, and in the PIH group $99.8 \pm 1.7 \mathrm{~mm} \mathrm{Hg}$ ( $P<0.0001$ vs controls). In the control group, 10 male and 13 female infants were born after $278.7 \pm 1.5$ days of gestation, with an average birthweight of $3285 \pm 99 \mathrm{~g}$, and in the PIH group, 14 male and 9 female infants after $274.8 \pm 3.3$ days of gestation $(P=0.243$ vs controls), who weighed on average $2969 \pm 153 \mathrm{~g}$ ( $P=0.046$ vs controls). In both groups, one woman gave birth by cesarean section. Although plasma antioxidant (Chapter 4) and phospholipid fatty acid ${ }^{15}$ levels change during pregnancy, values were not adjusted for gestational age, since the average duration of pregnancy was similar in both groups. 
Table 5.1. Plasma lipid-soluble antioxidant levels in women with uncomplicated pregnancies and women with pregnancies complicated with pregnancy-induced hypertension

control group

\begin{tabular}{|c|c|c|c|c|c|c|}
\hline & & & & \\
\hline & 3rd trimester & postpartum & change & 3rd trimester & postpartum & change \\
\hline Tocopherols & $42.42 \pm 1.24$ & $43.53 \pm 1.55$ & $+1.109 \pm 1.106$ & $43.04 \pm 2.38$ & $39.38 \pm 2.64$ & $-3.660 \pm 1.643^{\mathrm{a}, t}$ \\
\hline$\delta$-tocopherol & $0.19 \pm 0.02$ & $0.17 \pm 0.01$ & $-0.022 \pm 0.016$ & $0.22 \pm 0.03$ & $0.15 \pm 0.01$ & $-0.067 \pm 0.025^{a}$ \\
\hline B+ $\gamma$-tocopherol & $3.16 \pm 0.25$ & $2.78 \pm 0.22$ & $-0.380 \pm 0.172^{\mathrm{a}}$ & $3.44 \pm 0.38$ & $2.36 \pm 0.33$ & $-1.083 \pm 0.266^{b, t}$ \\
\hline$\alpha$-tocopherol & $39.06 \pm 1.31$ & $40.57 \pm 1.48$ & $+1.510 \pm 1.097$ & $39.38 \pm 2.26$ & $36.87 \pm 2.41$ & $-2.510 \pm 1.578^{\dagger}$ \\
\hline Carotenoids & $1.89 \pm 0.10$ & $1.85 \pm 0.11$ & $-0.040 \pm 0.073$ & $1.95 \pm 0.13$ & $1.59 \pm 0.14$ & $-0.356 \pm 0.113 \mathrm{~h}, t$ \\
\hline lutein & $0.64 \pm 0.04$ & $0.65 \pm 0.04$ & $+0.003 \pm 0.030$ & $0.66 \pm 0.05$ & $0.54 \pm 0.04$ & $-0.129 \pm 0.040^{b, t}$ \\
\hline B-cryptoxanthin & $0.31 \pm 0.03$ & $0.29 \pm 0.05$ & $-0.012 \pm 0.041$ & $0.34 \pm 0.05$ & $0.29 \pm 0.05$ & $-0.047 \pm 0.036$ \\
\hline lycopene & $0.62 \pm 0.05$ & $0.60 \pm 0.06$ & $-0.025 \pm 0.034$ & $0.65 \pm 0.05$ & $0.52 \pm 0.07$ & $-0.124 \pm 0.061^{\mathrm{a}}$ \\
\hline$\alpha$-carotene & $0.07 \pm 0.01$ & $0.07 \pm 0.01$ & $-0.001 \pm 0.008$ & $0.06 \pm 0.01$ & $0.05 \pm 0.01$ & $-0.010 \pm 0.003^{b}$ \\
\hline B-carotene & $0.25 \pm 0.03$ & $0.25 \pm 0.03$ & $-0.006 \pm 0.020$ & $0.24 \pm 0.02$ & $0.19 \pm 0.02$ & $-0.046 \pm 0.014^{b}$ \\
\hline phytofluene & $0.70 \pm 0.06$ & $0.66 \pm 0.05$ & $-0.045 \pm 0.041$ & $0.76 \pm 0.08$ & $0.60 \pm 0.10$ & $-0.160 \pm 0.101$ \\
\hline Retinol & $1.08 \pm 0.05$ & $1.06 \pm 0.07$ & $-0.023 \pm 0.073$ & $1.09 \pm 0.05$ & $0.99 \pm 0.07$ & $-0.099 \pm 0.073$ \\
\hline
\end{tabular}

Plasma lipid-soluble antioxidant levels $(\mu \mathrm{mol} / \mathrm{l})$ were studied in the 3rd trimester of pregnancy, and immediately postpartum, in women with uncomplicated pregnancies (control group, $n=23$ ), and women with pregnancies complicated with pregnancy-induced hypertension (PIH group, $n=23$ ). Phytofluene levels are reported as chromatogram peak areas (mV*min/ $\mu$ l plasma, amplification 100). Values are means $\pm S E M$.

$a, b$ Changes from the 3rd trimester to postpartum were examined by paired Student's t-tests: $a, P \leq 0.05 ; b, P<0.01$.

${ }^{\dagger}$ Differences between the control and PIH group for mean levels in the 3rd trimester, as well as for changes from the 3rd trimester to postpartum, were examined by paired Student's $\underline{\text { t-tests: }} P<0.05$. 
Table 5.2. Plasma phospholipid fatty acid composition and polyene unsaturation index in women with uncomplicated pregnancies and in women with pregnancies complicated with pregnancy-induced hypertension

control group

3rd trimester postpartum change

\begin{tabular}{l}
\hline Total fatty acids \\
saturates \\
monoenes \\
C18:1 n-9 \\
polyenes \\
n-6 \\
C18:2 \\
C20:4 \\
n-3
\end{tabular}

$\mathrm{n}-3$

C22:6

Polyene unsaluration index* absolute relative

$6238 \pm 155$
$2994 \pm 79$
$802 \pm 26$
$548 \pm 20$
$2442 \pm 61$
$2126 \pm 50$
$1373 \pm 37$
$478 \pm 21$
$296 \pm 15$
$222 \pm 12$

$6561 \pm 178$
$3192 \pm 86$
$838 \pm 30$
$577 \pm 25$
$2530 \pm 71$
$2199 \pm 68$
$1372 \pm 54$
$525 \pm 24$
$306 \pm 13$
$229 \pm 11$

$7235 \pm 211$

$116 \pm 1$

$\begin{array}{rrr}7591 \pm 219 & +356 \pm 180 \\ 116 \pm 1 & -0.1 \pm 0.8\end{array}$

PIH group

\begin{tabular}{|c|c|c|}
\hline 3rd trimester & postpartum & change \\
\hline $6389 \pm 241$ & $6147 \pm 343$ & $-242 \pm 234^{\dagger}$ \\
\hline $\begin{array}{r}3078 \pm 114 \\
797 \pm 48 \\
547 \pm 37\end{array}$ & $\begin{array}{r}3001 \pm 164 \\
803 \pm 50 \\
554 \pm 40\end{array}$ & $\begin{array}{l}-76 \pm 118 \\
+6 \pm 38 \\
+7 \pm 30\end{array}$ \\
\hline $2514 \pm 91$ & $2342 \pm 135$ & $-172 \pm 84 \neq$ \\
\hline $2175 \pm 80$ & $2015 \pm 116$ & $-160 \pm 74^{a, \neq}$ \\
\hline $1386 \pm 58$ & $1203 \pm 67$ & $-183 \pm 47^{c, f}$ \\
\hline $485 \pm 30$ & $510 \pm 39$ & $+26 \pm 23$ \\
\hline $317 \pm 16$ & $304 \pm 20$ & $-13 \pm 13$ \\
\hline $233 \pm 11$ & $231 \pm 13$ & $-2 \pm 9$ \\
\hline $7492 \pm 287$ & $7181 \pm 430$ & $-311 \pm 260^{\dagger}$ \\
\hline $117 \pm 1$ & $117 \pm 1$ & $-0.8 \pm 0.9$ \\
\hline
\end{tabular}

The fatty acid composition of plasma phospholipids $(\mu \mathrm{mol} / \mathrm{l})$ were studied in women with uncomplicated pregnancies (control), and women with pregnancies complicated with pregnancy-induced hypertension (PIH). Samples were collected as described in table 5.1 Values are means \pm SEM. * Calculated as the sum of the micromolar concentration of each polyene multiplied with its number of double bonds (absolute), and as the sum of the percentage of each polyene (moles/moles total fatty acids ${ }^{*} 100 \%$ ) multiplied with its number of double bonds (relative).

$a, b, c$ Changes from the 3rd trimester to postpartum were examined by paired Student's t-tests: $a, P<0.05 ; b, P<0.01 ; c, P<0.001$.

$t$. Differences between the control and PIH group for mean levels in the 3rd trimester, as well as for changes from the $3 r d$ trimester to postpartum, were examined by paired Student's t-tests: $t, P<0.05 ; \ddagger, P<0.01$. 
In the 3rd trimester of pregnancy, plasma antioxidant levels were similar in the PIH and control group (Table 5.1). In the control group, $B+\gamma$-tocopherol levels decreased on average by $5 \%(P=0.038)$ from the 3rd trimester to postpartum. Changes in levels of the other antioxidants were not statistically significant. In the PIH group, however, plasma levels of total tocopherol decreased on average by $8 \%(P=0.036), \delta$-tocopherol by $21 \%(P=0.013), B+\gamma$-tocopherol by $26 \%(P=0.0005)$, total carotenoid by $16 \%$ $(P=0.005)$, lutein by $15 \%(P=0.004)$, lycopene by $16 \%(P=0.052), \alpha$-carotene by $15 \%$ $(P=0.005)$, and $B$-carotene by $15 \%(P=0.003)$. The decrease in $B+\gamma$ tocopherol levels in the PIH group was significantly more pronounced than the decrease in $B+\gamma$-tocopherol levels in the control group $(P=0.042)$. The decreases in total tocopherol, total carotenoid, and lutein levels in the $\mathrm{PIH}$ group were also significantly different from the changes in the control group $(P=0.015, P=0.021$, and $P=0.013$, respectively). Although the changes in $\alpha$-tocopherol levels within each group did not reach statistical significance, the decrease in the $\mathrm{PIH}$ group was significantly different from the increase in the control group ( $P=0.024)$. However, no significant correlations were found between antioxidant levels and DBP, or between changes in these parameters from the 3rd trimester to postpartum.

Mean phospholipid fatty acid levels during the 3rd trimester were similar in the PIH and control group (Table 5.2). From the 3rd trimester to postpartum, total phospholipid fatty acid levels in the control group increased by $6 \%(P=0.049)$. This was due to an increase in saturated fatty acid levels of $7 \%(\mathrm{P}=0.015)$ and arachidonic acid (C20:4 n-6) levels of $10 \%(P=0.002)$. In the $\mathrm{PIH}$ group, phospholipid $n-6$ polyenes decreased by $8 \%(P=0.042)$, due to a decrease in linoleic acid $(C 18: 2 n-6)$ levels by $13 \%(P=0.0009)$. Significant differences between the change in the control group and change in the $\mathrm{PIH}$ group were noticed for phospholipid total fatty acid levels $(P=0.028)$, saturated fatty acid levels $(P=0.036)$, polyenes levels $(P=0.007), n-6$ polyenes levels $(P=0.009)$, and linoleic acid levels $(P=0.004)$. From the 3rd trimester to postpartum, the absolute polyene unsaturation index ( $\mathrm{Ul}_{\mathrm{abs}}$ ) increased by $5 \%$ in the control group $(P=0.061)$, and decreased by $5 \%$ in the $\mathrm{PIH}$ group $(P=0.245)$. These changes were significantly different between the two groups $(P=0.014)$. The relative polyene unsaturation index, however, did not change significantly from the 3rd trimester to postpartum. 
Table 5.3. Ratio of plasma lipid-soluble antioxidant levels to phospholipid absolute polyunsaturated fatty acid index in women with uncomplicated pregnancies and in women with pregnancies complicated with pregnancy-induced hypertension

control group

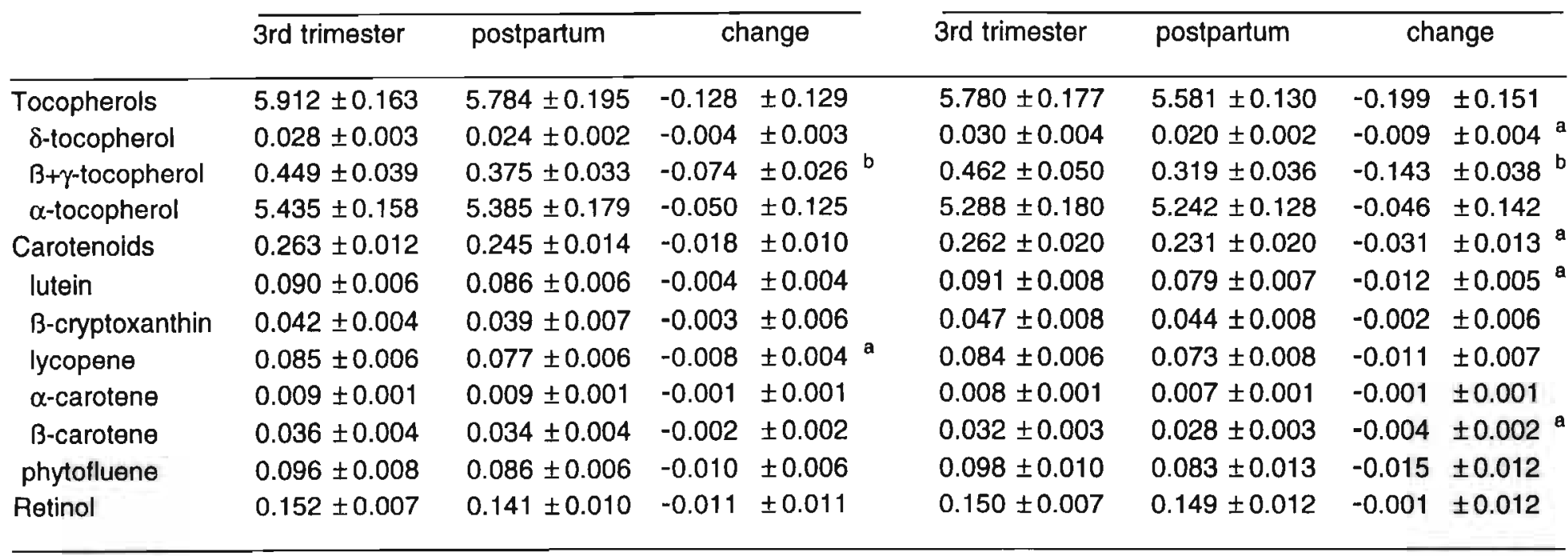

Ratios of plasma lipid-soluble antioxidant to phospholipid absolute polyunsaturated fatty acid index were studied in the 3rd trimester of pregnancy, and immediately postpartum, in women with uncomplicated pregnancies (control), and women with pregnancies complicaled with pregnancyinduced hypertension (PIH). Values are reported as means \pm SEM.

$a, b$ Changes from the 3rd trimester to postpartum were examined by paired Student's t-tests: $a, P<0.05 ; b, P<0.01$.

Differences between the control and PIH group for mean levels in the 3rd trimester, as well as for changes from the 3rd trimester to postpartum, were examined by paired Student's t-tests, but no significant differences were noticed. 
Table 5.4. Matemal and cord plasma lipid-soluble antioxidant levels after uncomplicated pregnancies and pregnancies complicated with pregnancy-induced hypertension

\begin{tabular}{|c|c|c|c|c|c|c|}
\hline & \multicolumn{3}{|c|}{ control group } & \multicolumn{3}{|c|}{ PIH group } \\
\hline & Maternal & Cord $^{\mathrm{a}}$ & $\mathrm{C} / \mathrm{M} \%$ & Maternal & Cord $^{\mathbf{a}}$ & $\mathrm{C} / \mathrm{M} \%$ \\
\hline Tocopherols & $43.53 \pm 1.55$ & $7.374 \pm 0.483$ & $17.2 \pm 1.4$ & $39.38 \pm 2.64$ & $7.860 \pm 0.602$ & $21.6 \pm 2.0$ \\
\hline$\delta$-tocopherol & $0.17 \pm 0.01$ & $0.053 \pm 0.006$ & $30.8 \pm 3.2$ & $0.15 \pm 0.01$ & $0.045 \pm 0.006$ & $30.0 \pm 2.6$ \\
\hline B+y-tocopherol & $2.78 \pm 0.22$ & $0.471 \pm 0.048$ & $18.1 \pm 1.8$ & $2.36 \pm 0.33$ & $0.479 \pm 0.041$ & $24.9 \pm 2.4^{b}$ \\
\hline$\alpha$-tocopherol & $40.57 \pm 1.48$ & $6.662 \pm 0.484$ & $16.7 \pm 1.4$ & $36.87 \pm 2.41$ & $7.477 \pm 0.584$ & $21.7 \pm 1.9$ \\
\hline Carotenoids & $1.85 \pm 0.11$ & $0.250 \pm 0.029$ & $14.0 \pm 1.3$ & $1.59 \pm 0.14$ & $0.259 \pm 0.023$ & $17.1 \pm 1.3$ \\
\hline lutein & $0.65 \pm 0.04$ & $0.131 \pm 0.028$ & $20.2 \pm 3.0$ & $0.54 \pm 0.04$ & $0.126 \pm 0.013$ & $23.7 \pm 1.7$ \\
\hline B-cryptoxanthin & $0.29 \pm 0.05$ & $0.043 \pm 0.004$ & $17.3 \pm 1.6$ & $0.29 \pm 0.05$ & $0.057 \pm 0.009$ & $20.4 \pm 1.3$ \\
\hline lycopene & $0.60 \pm 0.06$ & $0.041 \pm 0.003$ & $8.4 \pm 1.0$ & $0.52 \pm 0.07$ & $0.041 \pm 0.003$ & $9.6 \pm 0.9$ \\
\hline$\alpha$-carotene & $0.07 \pm 0.01$ & $0.008 \pm 0.001$ & $13.3 \pm 1.5$ & $0.05 \pm 0.01$ & $0.007 \pm 0.001$ & $17.0 \pm 1.7$ \\
\hline B-carotene & $0.25 \pm 0.03$ & $0.028 \pm 0.002$ & $13.4 \pm 1.6$ & $0.19 \pm 0.02$ & $0.027 \pm 0.002$ & $16.5 \pm 1.9$ \\
\hline phytofluene & $0.66 \pm 0.05$ & $0.000-0.005^{\star}$ & & $0.60 \pm 0.10$ & $0.000-0.010^{\star}$ & \\
\hline Retinol & $1.06 \pm 0.07$ & $0.617 \pm 0.056$ & $62.6 \pm 5.4$ & $0.99 \pm 0.07$ & $0.616 \pm 0.047$ & $73.1 \pm 9.8$ \\
\hline
\end{tabular}

After uncomplicated (control) pregnancy, and pregnancy complicated with pre(jnancy-induced hypertension (PIH), maternal and concurrent cord plasma were collected immediately after delivery, and analyzed for antioxidani levels $(\mu \mathrm{mol} / \mathrm{l})$. Phytofluene levels are reported as chromatogram peak areas $\left(\mathrm{mV}^{\star} \mathrm{min} / \mu \mathrm{l}\right.$ plasma, amplification 100$)$. C/M\% expresses the average ratio of cord vs maternal levels. Values are reported as means \pm SEM.

- Differences between cord and maternal plasma antioxidant levels within each group were examined by paired Student's t-tests, and all cord plasma antioxidant levels were significantly lower than maternal levels: $P<0.001$.

'Differences between control and PIH were examined by paired Student's t-tests: $P<0.05$.

* Cord plasma levels of phytofluene are given as ranges, since only small traces were detected in 11 control and $7 \mathrm{PIH}$ cord samples 
From the 3rd trimester to postpartum, ratios of $\beta+\gamma$-tocopherol and lycopene to $\mathrm{UI}_{\text {abs }}$ decreased by $9 \%(\mathrm{P}=0.010$ and $\mathrm{P}=0.044$, respectively) in the control group

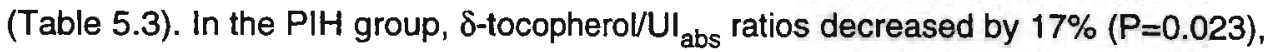
$B+\gamma$-tocopherol/UI $I_{a b s}$ by $23 \% \quad(P=0.001)$, lutein $/ U I_{a b s}$ by $11 \% \quad(P=0.022)$, and $B$-carotene/UI $\mathrm{abs}_{\text {by }} 9 \%(\mathrm{P}=0.046)$. However, these decreases did not significantly differ from the changes in the control group (Table 5.3).

Umbilical vein plasma antioxidant levels were significantly lower than matemal postpartum levels (Table 5.4), but no significant differences between the control group and $\mathrm{PIH}$ group were noticed. Cord/maternal ratios for tocopherols and carotenoids varied from $8 \%$ for lycopene to $31 \%$ for $\delta$-tocopherol levels, and except for $\delta$-tocopherol, these ratios were higher in the PIH group than in the control group. However, only the higher cord/matemal ratio for $B+\gamma$-tocopherol in the PIH group $(25 \%)$ reached statistical significance $(P=0.030)$ as compared with the ratio in the control group (18\%). This significant difference was mainly caused by the nonsignificant lower matemal plasma levels in the PIH group as compared with the control group, and hardly by a difference in cord plasma levels.

Total, saturated, monounsaturated, and n-6 polyunsaturated fatty acid levels in cord plasma phospholipids were similar after PIH and uncomplicated pregnancies (results not shown). Cord plasma n-3 polyunsaturated fatty acid levels in phospholipids were significantly higher after $\mathrm{PIH}(\mathrm{P}=0.005)$, mainly due to higher docosahexaenoic acid $(\mathrm{C} 22: 6 \mathrm{n}-3)$ levels $(\mathrm{P}=0.006)$. The relative polyene unsaturation index was also higher after $\mathrm{PIH}$ (142) than after uncomplicated (135) pregnancies $(\mathrm{P}=0.012)$.

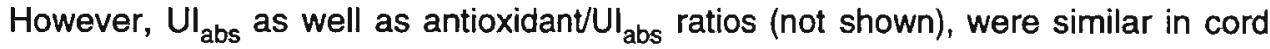
plasma from the PIH and control group.

\section{DISCUSSION}

In this study, we have examined 3rd trimester and postpartum antioxidant levels in plasma from women with pregnancies complicated with $\mathrm{PIH}$, and from women with uncomplicated pregnancies. In addition, umbilical vein levels in concurrent neonates were studied.

Mean 3rd trimester matemal plasma antioxidant levels were not significantly different between the PIH group and controls. Comparable vitamin E (total tocopherol) 
levels in women with mild PIH as compared with controls, were also reported by others $^{3,4}$. In women with severe $\mathrm{PIH}$, decreased ${ }^{3}$ as well as increased plasma vitamin $\mathrm{E}$ levels ${ }^{4}$ have been reported. Power analysis showed that the number of subjects in the present study were sufficient to detect differences as reported in these latter studies ${ }^{3,4}$. However, in our study, only 4 of 23 women with PIH had a maximum DBP of 110 or more, one of the criteria for severe PIH in those studies ${ }^{3,4}$. Therefore, our study cannot be compared with these studies, and the discrepancy with respect to vitamin E levels in women with severe PIH remains to be investigated. Another research group also reported that vitamin E levels in women with PIH were decreased as compared with controls. However, in one study ${ }^{8}$ average plasma vitamin $E$ levels in $\mathrm{PIH}$ and controls were $6.3 \mu \mathrm{mol} / \mathrm{l}$ and $11.4 \mu \mathrm{mol} /$, respectively, whereas in a recent study ${ }^{16}$ levels were 46.5 and $88.0 \mu \mathrm{mol} / \mathrm{l}$, respectively. This large discrepancy between these studies, and with the present study, limits the use of these results for comparison. Uotila et al. ${ }^{4}$ considered the possibility that vitamin $E$ levels may not reflect general antioxidant status, and that a shortage of other antioxidants might prevail in lipid compartments. Our study, however, also showed no difference in plasma levels of various other lipid-soluble antioxidants during the 3rd trimester in complicated and uncomplicated pregnancies. These result are consistent with previous reports on B-carotene levels in $\mathrm{PIH}^{8,9}$. Plasma retinol levels were also similar in the two groups during the 3rd trimester, which does not agree with results from Jendryczko et al. ${ }^{8}$, who reported that retinol levels may be decreased throughout pregnancy in women with PIH.

In the present study, several antioxidant levels decreased from the 3rd trimester to postpartum in the PIH group, whereas only $\beta+\gamma$ tocopherol levels decreased in the control group. The decrease in plasma $\beta+\gamma$-tocopherol levels was also found in another group of women with uncomplicated pregnancies studied at our department (Chapter 4). In agreement with the present study, plasma levels of other lipid-soluble antioxidants also did not change from the 3rd trimester to postpartum during uncomplicated pregnancy in the previous study, except for $\delta$-tocopherol levels which decreased (Chapter 4). Although the decrease in $\beta+\gamma$-tocopherol levels may be a result of (oxidative) stress during labor, the period between 3rd trimester sampling and partus is too long to substantiate this. Furthermore, the reason why only B+ $\psi$-tocopherol levels decrease from the 3rd trimester to postpartum, and levels of other lipid-soluble antioxidants do not, is unclear. However, in patients undergoing 
percutaneous transluminal coronary angioplasty, a medical procedure associated with oxidative stress, we also noticed a decrease in concentrations of the less abundant tocopherol isomers in low density lipoproteins (LDL), 2 days after the procedure, but no decrease in LDL $\alpha$-tocopherol concentrations (Chapter 6).

The decreases in $\beta+\gamma$-tocopherol, total tocopherol, $\alpha$-tocopherol, total carotenoid, and lutein levels from the 3rd trimester to postpartum in the PIH group were significantly different from the changes in the control group. However, at the same time, the peroxidation-susceptible polyunsaturated fatty acids in phospholipids, and thus the $\mathrm{Ul}_{\text {abs, }}$, also decreased, as compared with the control group. Nonetheless,

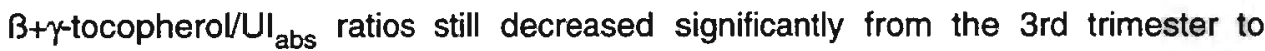
postpartum in both groups, which is consistent with previous results (Chapter 4). In the control group, lycopene/ $\mathrm{Ul}_{\text {abs }}$ ratios also decreased from the 3rd trimester to postpartum. Furthermore, within the PIH group, ratios for several antioxidants decreased significantly, despite the decreased in $\mathrm{UI}_{\text {abs, }}$, although these decreases were not significantly different from changes in the control group. Possibly, a future study with more cases of severe PIH may show whether the decreases in maternal

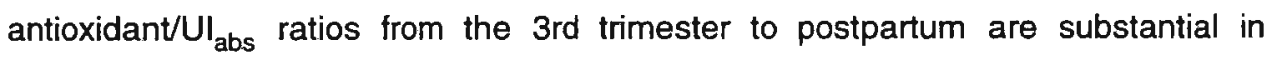
pregnancies complicated with $\mathrm{PIH}$.

Results from this study do not indicate that the status of lipid-soluble antioxidants in the neonate is affected by PIH complications in the mother, since cord plasma antioxidant levels and antioxidant/UI $\mathrm{I}_{\text {abs }}$ ratios in both groups were similar. For vitamin $\mathrm{E}$, these findings agree with previous reports ${ }^{6,7}$, but Uotila et $a l^{6}{ }^{6}$, have found lower cord plasma retinol levels after pregnancies complicated with PIH.

Vitamin $E$ is regarded as one of the most important antioxidants in lipophilic compartments, like membranes. However, the water-soluble antioxidant vitamin $\mathrm{C}$ may have a sparing effect on vitamin $E$ by regenerating the oxidized form ${ }^{17,18}$. Although Mikhail et al. ${ }^{9}$ found decreased plasma levels of vitamin $C$ during the 3rd trimester in women with $\mathrm{PIH}$, Uotila et al..$^{5}$ reported similar levels just before delivery in women with and without $\mathrm{PIH}$. In women with $\mathrm{PIH}$, Uotila et al..$^{5}$ also found increased plasma levels of uric acid and unidentified antioxidants, both contributing to higher TRAP levels. Conversely, Davidge et al. ${ }^{2}$ reported a lower antioxidant capacity of serum to inhibit autoxidation of brain homogenates in women with $\mathrm{PIH}$, and they suggested that the increase in serum antioxidant capacity, as seen during uncomplicated pregnancies, is absent in women with PIH. However, in the present study, 3rd trimester plasma 
tocopherol levels in the PIH group were consistent with increases seen during uncomplicated pregnancies (Chapter 4), and plasma vitamin $C$ levels in women with or without PIH are similarly increased as compared with nonpregnant controls ${ }^{5}$.

In summary, this study showed that matemal plasma lipid-soluble antioxidant levels during the 3rd trimester were similar in pregnancies complicated with mild PIH and uncomplicated pregnancies. From the 3rd trimester to postpartum, however, plasma levels of several antioxidants decreased in the PIH group as compared with controls. At the same time, plasma unsaturation, as estimated by the phospholipid polyene unsaturation index, decreased as well. Nonetheless, the PIH group showed significant

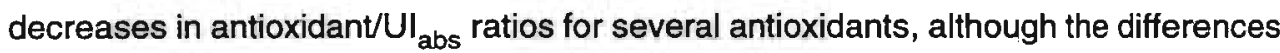
with changes in the control group did not reach statistical significance. Neonatal lipidsoluble antioxidant levels in plasma were unaffected by maternal PIH.

\section{REFERENCES}

1. Walsh SW. Lipid peroxidation in pregnancy. Hypertens Pregn 1994; 13:1-32.

2. Davidge ST, Hubel CA, Brayden RD, Capeless EC, McLaughlin MK. Sera antioxidant activity in uncomplicated and preeclamptic pregnancies. Obstet Gynecol 1992; 79:897-901.

3. Wang YP, Walsh SW, Guo JD, Zhang JY. The imbalance between thromboxane and prostacyclin in preeclampsia is associated with an imbalance between lipid peroxides and vitamin $E$ in matemal blood. Am J Obstet Gynecol 1991; 165:1695-1700.

4. Uotila JT, Tuimala RJ, Aarnio TM, Pyykkō KA, Ahotupa MO. Findings on lipid peroxidation and antioxidant function in hypertensive complications of pregnancy. $\mathrm{Br} \mathrm{J}$ Obstet Gynaecol 1993; 100:270-276.

5. Uotila JT, Kirkkola AL, Rorarius M, Tuimala RJ, Metsä-Ketelà T. The total peroxyl radical-trapping ability of plasma and cerebrospinal fluid in normal and preeclamptic parturients. Free Rad Biol Med 1994; 16:581-590.

6. Uotila J, Tuimala R, Pyykkō K, Ahotupa M. Pregnancy-induced hypertension is associated with changes in matemal and umbilical blood antioxidants. Gynecol Obstet Invest 1993; 36:153-157.

7. von Mandach U, Huch $R$, Huch A. Matemal and cord serum vitamin $E$ levels in normal and abnormal pregnancy. Int J Vitam Nutr Res 1993; 63:26-32.

8. Jendryczko $A$, Drożdż $M$. Plasma retinol, $\beta$-carotene and vitamin $E$ levels in relation to the future risk of pre-eclampsia. Zent BI Gynākol 1989; 111:1121-1123.

9. Mikhail MS, Anyaegbunam A, Garfinkel D, Palan PR, Basu J, Romney SL. Preeclampsia and antioxidant nutrients: decreased plasma levels of reduced ascorbic acid, alpha-tocopherol, and beta-carotene in women with preeclampsia. Am J Obstet Gynecol 1994; 171:150-157.

10. Roberts JM, Taylor RN, Musci JT, Rodgers GM, Hubel CA, McLauglin MK. Preeclampsia: an endothelial cell disorder. Am J Obstet Gynecol 1989; 161:1200-1204. 
11. van der Schouw YT, Al MDM, Hornstra G, Bulstra-Ramakers MTEW, Huisjes HJ. Fatty acid composition of serum lipids of mothers and their babies after normal and hypertensive pregnancies. Prostaglandins Leukot Essent Fatty Acids 1991; 44:247-252.

12. Halliwell $B$, Gutteridge JMC. The importance of free radicals and catalytic metal ions in human diseases. Mol Aspects Med 1985; 8:89-193.

13. AI MD, Houwelingen AC v, Badart-Smook A, Hasaart TH, Roumen FJ, Homstra G. The essential fatty acid status of mother and child in pregnancy-induced hypertension: a prospective longitudinal study. Am J Obstet Gynecol 1995; 172:1605-1614.

14. Davey DA, MacGillivray 1. The classification and definition of the hypertensive disorders of pregnancy. Am J Obstet Gynecol 1988; 158:892-8.

15. Al MDM, Houwelingen AC v, Kester ADM, Hasaart THM, de Jong AEP, Hornstra G. Matemal essential fatty acid pattems during normal pregnancy and their relationship to the neonatal essential fatty acid status. Br J Nutr 1995; 74:55-68.

16. Jendryczko A, Tomala J. The total free radical trapping ability of blood plasma in eclampsia. Zent BI Gynākol 1995; 117:126-129.

17. Niki E. Antioxidants in relation to lipid peroxidation. Chem Phys Lipids 1987; 44:227-253.

18. Stocker R, Frei B. Endogenous antioxidant defences in human blood plasma. In: Sies H (ed.). Oxidative Stress. Oxidants and Antioxidants. London: Academic Press, 1991:213-243. 


\section{Percutaneous transluminal coronary angioplasty as model for lipid peroxidation-associated oxidative stress in humans}

Gerard S. Oostenbrug, Ronald P. Mensink, Frits W.H.M. Bär \& Gerard Hornstra

Based on: Free Radical Biology in Medicine (in press).

\section{ABSTRACT}

Animal studies have suggested that myocardial ischemia/reperfusion causes oxidative stress. We therefore examined whether routinely performed percutaneous transluminal coronary angioplasty (PTCA) might be a human ischemia/reperfusion model for oxidative stress-induced lipid peroxidation. Fasting antecubital venous blood was sampled from 13 patients on the moming of PTCA, and 2 days after PTCA. Venous and coronary arterial blood were sampled just before and 10 minutes after the first balloon inflation. Samples were analyzed for plasma and LDL lipid hydroperoxide levels, in vitro oxidation of LDL, and LDL antioxidant levels. Lipid hydroperoxide levels in plasma and LDL remained unchanged throughout the study. During the first 10 minutes of PTCA, the lagtime during oxidation of LDL in vitro did not change, but the maximum rate of oxidation decreased in venous and arterial samples (Wilcoxon signed rank test: $P<0.002$ ). At the same time, total tocopherol levels in LDL significantly increased by $6.3 \%(P=0.048)$ in arterial, but not in venous samples. Total carotenoid levels increased by $3.8 \%(P=0.127)$ in arterial samples and decreased by $2.9 \%$ $(P=0.040)$ in venous samples. Forty hours after PTCA, LDL oxidation parameters and LDL antioxidant levels were similar to baseline, except for about $17 \%$ lower levels of $\delta$-tocopherol $(P=0.037)$ and $\gamma$-tocopherol $(P=0.014)$. Our results, therefore, do not support that $P T C A$ in humans is associated with oxidative stress-induced lipid peroxidation. 


\section{INTRODUCTION}

Animal studies have suggested that, after a period of ischemia, the reintroduction of oxygen during reperfusion of the heart causes oxidative stress. Oxygen-derived free radicals, for example, were detected during myocardial reperfusion after mechanically induced ischemia in isolated rabbit ${ }^{1}$ and rat $^{2}$ hearts, and open-chest dogs ${ }^{3}$. These hazardous free radicals can interact with polyunsaturated fatty acids (PUFAs) ${ }^{4}$, thereby generating lipid hydroperoxides and aldehydes, which may cause cellular damage ${ }^{5,6}$. Evidence for free radical and lipid peroxidation-associated oxidative stress is supported by studies showing that antioxidants and antioxidant enzymes reduce ischemia/reperfusion injury in ex vivo organ and in vivo animal studies ${ }^{7,8}$. However, measurement of parameters of lipid peroxidation in isolated animal hearts during ischemia/reperfusion are conflicting ${ }^{9,10}$.

Oxidative stress following ischemia/reperfusion in humans has mainly been investigated during surgery under variable conditions ${ }^{11,12}$. Percutaneous transluminal coronary angioplasty (PTCA) is the most routinely used technique for mechanical revascularization of obstructed coronary arteries, and surgical conditions are often well-standardized. Therefore, PTCA may present a usable model for oxidative stress in humans. Indeed, free radicals were detected during PTCA in human plasma samples ${ }^{13,14}$.

Lipid peroxidation-associated oxidative stress during PTCA in humans has usually been demonstrated by measuring thiobarbituric acid reactive substances (TBArs) in plasma ${ }^{15,16}$. The TBArs assay measures malondialdehyde (MDA), one of the many degradation products of lipid hydroperoxides. Although improved in recent years, the low specificity of this assay for MDA, the numerous non-lipid TBA-positive materials, and the fact that part of the MDA is formed in vitro due to the extreme conditions needed for the reaction of MDA with TBA, have limited the value of this assay for quantifying lipid peroxidation (reviewed in Ref. 17). Furthermore, not all studies found increased levels of TBArs after PTCA ${ }^{18,19}$. Therefore, we decided to investigate lipid peroxidation during and after PTCA using an assay directly measuring lipid hydroperoxides in plasma under moderate conditions. In addition, interaction of free radicals with PUFAs in low density lipoproteins (LDLs) may lead to oxidatively modified LDL, and detection of modified LDL may indicate oxidative stress. Therefore, oxidative modification of LDL was investigated as well, by measuring its lipid hydroperoxide and antioxidant content, and its susceptibility to oxidation in vitro. 


\section{PATIENTS AND METHODS}

\section{Patients and procedure}

Thirteen patients ( 1 female, 12 males) with stable angina pectoris enrolled in this study after angiographically detected occlusion of a coronary artery. All regular medications were continued, including acetylsalicylic acid, calcium antagonists, Badrenoceptor antagonists, and nitrates. Informed consent was received from each patient, and prior approval for the study was obtained from the Medical Ethics Committee of the University of Limburg.

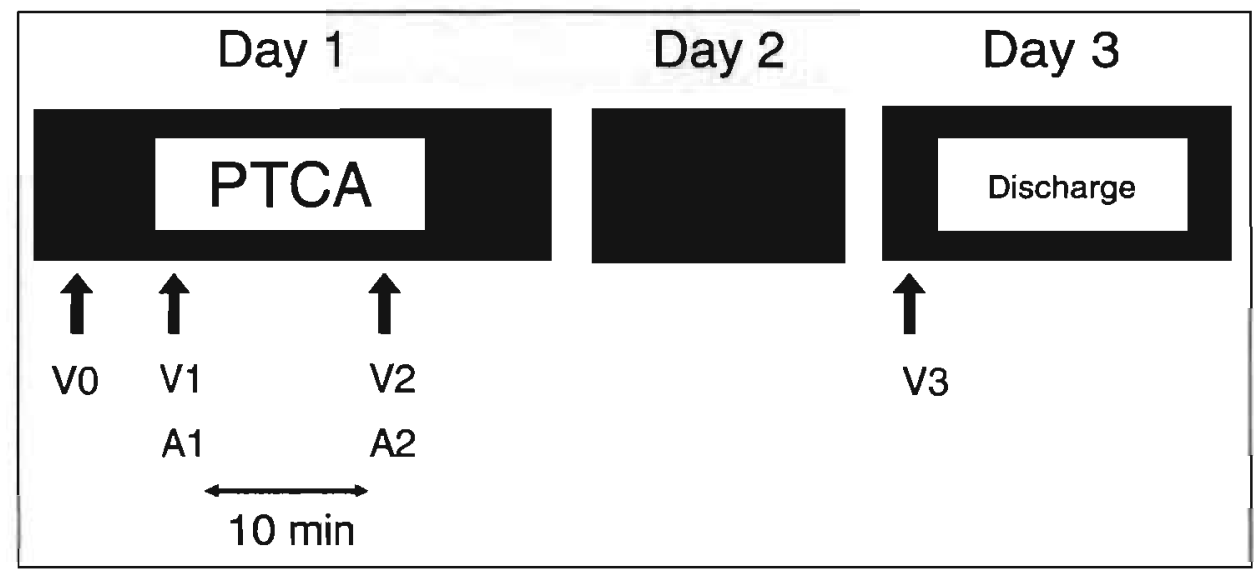

Figure 6.1. Design of the study. Thirteen patients underwent PTCA according to a standard protocol. Venous blood was sampled on the moming of PTCA (VO) and two days thereafter (V3). Just before PTCA, venous (V1) and anterial (A1) blood was sampled, and repeated ten minutes after the first balloon inflation (V2 and A2). During these ten minutes, 1 to 3 dilations were performed, and the procedure was completed for 10 patients. In two patients a fourth angioplasty was performed after these ten minutes, and for one patient a total of 12 dilations was necessary.

On the moming of PTCA, a fasting venous blood sample was taken from a forearm (V0), one hour after oral administration of $80 \mathrm{mg}$ acetylsalicylic acid. Four to seven hours after V0, PTCA was performed according to a standard protocol. Preceding PTCA, local anaesthesia with lidocaine was given. The catheter was 
inserted by the femoral route. After administration of heparin (i.v. 10,000 IU), a venous sample (V1) was taken from a intravascular teflon cannula, which was placed in an antecubital vein during the entire procedure. At the same time, an arterial sample proximal to the lesion (A1) was taken from the coronary guiding catheter. Then, nitroglycerin (i.c. $200 \mu \mathrm{g}$ ) was administered, and the first balloon inflation was started. Additional nitroglycerin was given during the procedure if cardiac instability occurred as indicated by electrocardiography. Ten minutes after the first balloon inflation, again a venous and an arterial sample were taken (V2 and A2). During these ten minutes, the PTCA procedure was continued according to normal routine protocol. In 10 of 13 patients the procedure was completed within these ten minutes. After the procedure, patients were kept under observation, and received standard hospital meals during this period. Approximately 42 hours after PTCA ( 48 hours after V0), a final fasting venous blood sample was drawn from a fore-arm (V3), again one hour after oral administration of $80 \mathrm{mg}$ acetylsalicylic acid. The design is schematically presented in Figure 6.1. Two patients were discharged from the hospital before a V3-sample was taken. One patient died the night before the V3-sampling.

\section{Blood collection}

Seven $\mathrm{ml}$ of blood were collected into decapped Monoject-tubes (Sherwood Medical, Ballymoney, Northem Ireland) containing $15 \mathrm{mg}$ ethylenediaminetetraacetic acid, tripotassium salt $\left(K_{3} E D T A\right)$. Tubes were immediately closed after collection, and blood and EDTA were carefully mixed. Plasma was obtained after 15 minutes of centrifugation at $2000 \mathrm{~g}$ and $4{ }^{\circ} \mathrm{C}$, within one hour of collection. Plasma for lipid hydroperoxide analysis was stored at $-80^{\circ} \mathrm{C}$.

\section{Blood analyses}

Plasma and $L D L$ lipid hydroperoxides

Lipid hydroperoxide levels in plasma and LDL (see below) were analyzed with a commercially available kit, based on the reaction with a methylene blue derivative (MCDP) (Determiner LPO-CC, Kamiya Biomedical Company, Thousand Oaks, CA, 
USA). The reaction is catalyzed by hemoglobin added to the reaction mixture, and the formation of methylene blue is measured spectrophotometrically at $675 \mathrm{~nm}$. The method was modified to measure low concentrations, and analyses were performed using a COBAS BIO centrifugal analyzer ( $F$. Hoffmann-La Roche, Basel, Switzerland). Briefly, $12 \mu \mathrm{l}$ of plasma were mixed with $75 \mu \mathrm{l}$ of reagent 1 , containing ascorbic oxidase and lipoprotein lipase. After incubation for 6 minutes at $30^{\circ} \mathrm{C}, 130 \mu$ of reagent 2 (MCDP and hemoglobin) were added. Absorbance at $675 \mathrm{~nm}$ was measured after another 12 minutes of incubation at $30^{\circ} \mathrm{C}$. The analyzer was completely shielded from light to prevent destabilization of the reagents. A hydroperoxide calibration curve was obtained by diluting the supplied cumene hydroperoxide standard solution (50 $\mu \mathrm{mol} / \mathrm{l})$ to 1,2 and $3 \mu \mathrm{mol} / \mathrm{l}$ with distilled water. These calibration samples were present in each run. All plasma samples were analyzed in duplicate. The between-run coefficient of variation for the 3 calibration samples ranged from $8.6 \%$ to $10.2 \%$ (14 runs). The average difference between duplicate measurements of plasma samples ( $n=104$ ) was $9.0 \pm 9.5 \%$. Levels were lipid-standardized $(\mu \mathrm{mol} / \mathrm{mmol}$ cholesterol) using cholesterol levels.

\section{$L D L$ isolation and oxidation}

LDL was isolated from fresh plasma immediately after collection by single spin density gradient ultracentrifugation ${ }^{20}$. Sudan Black $B$ to prestain the lipoproteins, however, was not added because it interfered with the LDL oxidation analysis. All gradient solutions contained $1.0 \mathrm{~g} / \mathrm{L}$ of $\mathrm{Na}_{2}$ EDTA. $2 \mathrm{H}_{2} \mathrm{O}$ to prevent initiation of oxidation of the LDL particle during isolation. The 5 samples from each patient collected on the same day (VO, A1, V1 , A2, V2) were centrifuged in the same run. LDL ( $\rho=1.019-1.055$ $\mathrm{g} / \mathrm{ml}$ ) was collected by aspiration, and $0.6 \mathrm{ml}$ was stored at $-80^{\circ} \mathrm{C}$ for analysis of antioxidants and lipid hydroperoxides. The remaining $L D L$ was used for oxidation studies: $L D L$ of the first 5 samples ( $V 0, A 1, V 1, A 2, V 2)$ was stored under a nitrogen atmosphere for two days at $4{ }^{\circ} \mathrm{C}$, and then processed together with the sixth sample (V3). EDTA was removed from LDL samples by gel filtration. To this end, $1.3 \mathrm{ml}$ of LDL was diluted with $1.5 \mathrm{ml}$ nitrogen-purged phosphate-buffered saline (PBS: 10 $\mathrm{mmol} / \mathrm{K} \mathrm{KH}_{2} \mathrm{PO}_{4}, 0.15 \mathrm{~mol} / \mathrm{l} \mathrm{NaCl}, \mathrm{pH}$ 7.4). Then, two PD-10 Sephadex G25-M gel filtration columns (Pharmacia, Roosendaal, The Netherlands) were placed on top of each other and equilibrated with nitrogen-purged PBS. Finally, $2.5 \mathrm{ml}$ of the LDLdilution was applied to the upper column and eluted with $5.5 \mathrm{ml}$ nitrogen-purged PBS 
in two volumes of $2.75 \mathrm{ml}$. The last eluent of $2.75 \mathrm{ml}$, containing the EDTA-free LDL, was kept under a nitrogen atmosphere. A previous experiment showed that the percentage removal of EDTA using this method was approximately $99.8 \%$. The concentration of EDTA was determined by mixing $5 \mu \mathrm{l}$ of LDL eluent with $390 \mu \mathrm{l} 0.33$ $\mathrm{mmol} / /$ iron-phenanthroline. Iron-phenanthroline was freshly prepared by adding $\mathrm{FeSO}_{4} .7 \mathrm{H}_{2} \mathrm{O}$ to 1,10-phenanthroline monohydrochloride monohydrate in PBS. After 1 hour of incubation at $25^{\circ} \mathrm{C}$, the decrease in absorbance at $510 \mathrm{~nm}$, due to the competition of EDTA with iron for the phenanthroline molecule, was compared with that of standard solutions of EDTA in PBS. Before and after the gelfiltration, cholesterol concentrations in LDL were determined, using a enzymatic colorimetric test kit (Monotest Cholesterol, Boehringer Mannheim, Mannheim, Germany). Within 15 minutes after gelfiltration, samples were diluted with PBS (not purged with nitrogen) to a final concentration of $0.26 \mathrm{mmol}$ cholesteroll, and oxidation was initiated with $\mathrm{CuCl}_{2}$ (final concentration: $15 \mu \mathrm{mol} / \mathrm{l}$ ). Oxidation of PUFAs was measured spectrophotometrically by monitoring the formation of conjugated fatty acid dienes at $37^{\circ} \mathrm{C}$ in a quartz cuvette. The change in absorbance at $234 \mathrm{~nm}$ was recorded over a 4-hour period, and the amount of dienes was calculated using the molar extinction coefficient for conjugated dienes $\left(\varepsilon_{234}=29,500 \mathrm{Vmol} / \mathrm{cm}\right)^{21}$.

\section{LDL antioxidants}

LDL was analyzed for levels of tocopherols and carotenoids by a method slightly modified from Hess et al. ${ }^{22}$. LDL $(250 \mu \mathrm{l})$ was mixed for 10 seconds with retinol acetate $(25 \mu \mathrm{l}, 4.5 \mathrm{mg} / \mathrm{l})$ as intemal standard, distilled water $(250 \mu \mathrm{l})$, ethanol $(250 \mu \mathrm{l})$ and methanol $(250 \mu \mathrm{l})$. Then $\mathrm{n}$-hexane $(1 \mathrm{ml})$ was added, the samples were shaken for 10 minutes, and centrifuged at $2000 \mathrm{~g}$ for 10 minutes. The organic top phase was removed and the extraction procedure repeated. The two hexane phases were pooled, and evaporated to dryness under nitrogen. Residues were redissolved in $60 \mu \mathrm{l}$ ethanol/dioxane (1:1) by shaking for 10 minutes, and then $90 \mu$ acetonitrile were added. One hundred microliters were injected (Promis II injection system; Spark Holland, Emmen, The Netherlands) on a Inertsil ODS-2, $5 \mu \mathrm{m} \mathrm{C-18,} 50 \mathrm{~mm} * 4.6 \mathrm{~mm}$ I.D. precolumn, followed by a Inertsil ODS-2, $5 \mu \mathrm{m} \mathrm{C-18,150} \mathrm{mm} \mathrm{*} 4.6 \mathrm{~mm}$ I.D. column (GL Sciences, Tokyo, Japan), and eluted with $68 \%$ acetonitrile/22\% tetrahydrofuran $7 \%$ methanol/3\% ammonium acetate $(10 \mathrm{~g} / \mathrm{l})$ at a flow rate of 1.0 mVmin (Gynkotek model 300 pump; Gynkotek, Germering, Germany). The mobile 
phase was passed through a $0.2 \mu \mathrm{m}$ regenerated cellulose filter (Sartorius, Goettingen, Germany) before use, and degassed on-line using a GT-103 metal free degassing device (Separations, H.I. Ambacht, The Netherlands). All samples from each patient were analyzed in the same analytical run. Detection of carotenoids at 450 $\mathrm{nm}$ was carried out with an Applied Biosystems 785A absorbance detector (Applied Biosystems, Maarssen, The Netherlands). Simultaneously, fluorescence detection (Jasco 821-FP; Japan Spectroscopic Company, Hachioji City, Japan) was used for detection of retinol acetate (excitation at $330 \mathrm{~nm}$, emission at $470 \mathrm{~nm}$ : 0-6.5 min), tocopherol isomers (excitation at $298 \mathrm{~nm}$, emission at $328 \mathrm{~nm}: 6.5-13 \mathrm{~min}$ ) and phytofluene (excitation at $349 \mathrm{~nm}$, emission at $480 \mathrm{~nm}$ : $13-23 \mathrm{~min})^{22}$. Peak areas were calculated with the Gynkosoft Chromatography Data System (Gynkotek, Germany), and calibrated against a mixture of the various standard substances in ethanol/dioxane/acetonitrile (1:1:3). The concentration of the separate standard solutions was determined spectrophotometrically using molar extinction coefficients reported in literature $23,24,25$. Molar extinction coefficients used were for: retinol acetate 50.9 (326 nm), $\delta$-tocopherol $3.52(298 \mathrm{~nm}), B$ and $\gamma$-tocopherol $3.81(298 \mathrm{~nm}), \alpha$-tocopherol $3.26(292 \mathrm{~nm})$, lutein $128(445 \mathrm{~nm})$, canthaxanthin $127(470 \mathrm{~nm})$, cryptoxanthin 131 (452 nm), lycopene $185(472 \mathrm{~nm}), \alpha$-carotene $150(440 \mathrm{~nm})$, and B-carotene 137 $\mathrm{V} / \mathrm{mmol} \cdot \mathrm{cm}$ (ethanolic solutions). Since pure phytofluene was not available, quantitative determination of this compound was not possible. B-Tocopherol co-eluted with $\gamma$-tocopherol. Previous reports on B-tocopherol levels in plasma vary between 'negligible ${ }^{, 22}, 9 \%^{26}$ or $20 \%{ }^{27}$ of $\gamma$-tocopherol levels. B-Tocopherol levels in LDL are unknown. The canthaxanthin standard eluted separately from the lutein standard, but in LDL samples canthaxanthin concentrations were too low to appear as a separate peak. Therefore, concentrations of lutein reported here may also include canthaxanthin. Levels of LDL antioxidants were lipid-standardized $(\mathrm{nmol} / \mathrm{mmol}$ cholesterol) using LDL cholesterol levels. Tocopherol ( $\alpha, \beta, \gamma$ and $\delta), B$-cryptoxanthin, lycopene, and $\alpha$ - and $B$-carotene standards were a generous gift from $F$. Hoffmann-La Roche (Basel, Switzerland). Lutein was obtained from Sigma Chemical Company (Saint Louis, USA), and canthaxanthin from Fluka Chemie (Bomem, Belgium). Retinol acetate and all other chemicals were purchased from Merck (Darmstadt, Gemany). Acetonitrile, tetrahydrofuran and methanol used for HPLC were of HPLC grade. 


\section{Statistics}

Short-term (V2 minus V1; A2 minus A1) and longer-term (V3 minus V0) effects of PTCA, as well as differences in short-term effects between arterial and venous samples (V2-V1 minus A2-A1) were examined by Wilcoxon signed rank tests (Statistical Analysis System, SAS Institute Inc., 1989). Spearman correlation (r) coefficients were calculated between values in venous (V1) and arterial (A1) samples, and differences were compared by Wilcoxon signed rank tests. Spearman correlation coefficients were also computed to assess the relationship between lipid hydroperoxide levels in plasma and LDL, and between various parameters when applicable. Nonparametric tests were chosen because of the relatively small number of subjects. Results are given as means \pm SD.

\section{RESULTS}

\section{Basic and clinical characteristics}

The 13 patients were between 43 and 71 years of age (mean: 58 years), and between 155 and $190 \mathrm{~cm}$ tall (mean: $174 \mathrm{~cm}$ ). Body weight ranged from 59 to $95 \mathrm{~kg}$ (mean: $80 \mathrm{~kg}$ ), and body mass index from 20 to $30 \mathrm{~kg} / \mathrm{m}^{2}$ (mean: $26 \mathrm{~kg} / \mathrm{m}^{2}$ ). Five patients did not smoke, two had stopped smoking, and six smoked on average 19 cigarettes per day (range: 7-30 cigarettes). The average length of the coronary artery lesions was $11 \mathrm{~mm}$ (range: 2 to $20 \mathrm{~mm}$ ), causing a $90 \%$ (range: 80 to $99 \%$ ) narrowing of the affected arteries, as determined by qualitative assessment. Eight patients required PTCA in the left anterior descending artery (LAD), one patient in the right coronary artery (RCA), and four in the circumflex branch (RCX). One balloon inflation was performed in 4 patients, two in 4 patients, three in 2 patient, four in 2 patients, and twelve dilations in 1 patient. Each dilation was sustained for on average 1 minute. After PTCA, the average degree of stenosis was reduced to $21 \%$ (range: 5 to $70 \%$ ). There was no apparent relation between the clinical characteristics and lipid hydroperoxide levels, LDL oxidation in vitro, or LDL antioxidant levels. No significant baseline differences were noticed between samples from the 5 non-smokers and the 
6 smokers, except for lower levels of LDL tocopherols in smokers ( $3468 \pm 319$ versus $4357 \pm 445 \mathrm{nmol} / \mathrm{mmol}$ cholesterol in non-smokers, Wilcoxon rank-sum test: $P=0.046$ ). Responses to PTCA as described below, however, were similar in smokers and nonsmokers.

\section{Short-term effects of PTCA}

Plasma and LDL lipid hydroperoxides

Table 6.1. Short-term effect of PTCA on plasma and LDL lipid hydroperoxide levels, and LDL oxidation in vitro in venous and arterial samples.

$\begin{array}{lrrr}\text { Sample } & \begin{array}{r}\text { V1 or } A 1 \\ \left(n=13^{\mathrm{a}}\right)\end{array} & \begin{array}{r}\text { V2 or } A 2 \\ \left(n=13^{\mathrm{a}}\right)\end{array} & \begin{array}{c}\text { Change } \\ (\Delta V, \Delta A)\end{array}\end{array}$

Lipid hydroperoxides ( $\mu \mathrm{mol} / \mathrm{mmol}$ cholesterol)

$\begin{array}{llllr}\text { Plasma } & \text { venous } & 0.24 \pm 0.17 & 0.30 \pm 0.55 & 0.07 \pm 0.40 \\ & \text { arterial } & 0.32 \pm 0.52 & 0.25 \pm 0.21 & -0.07 \pm 0.38 \\ \text { LDL } & \text { venous } & 0.95 \pm 0.23 & 1.02 \pm 0.25 & 0.07 \pm 0.19 \\ & \text { arterial } & 1.03 \pm 0.25 & 0.98 \pm 0.23 & -0.05 \pm 0.17\end{array}$

LDL oxidation

$\begin{array}{llrrr}\text { Lagtime } & \text { venous } & 62.1 \pm 11.1 & 63.0 \pm 9.9 & 0.91 \pm 3.83 \\ \text { (min) } & \text { arterial } & 61.7 \pm 10.6 & 61.0 \pm 12.4 & -0.77 \pm 6.12 \\ \text { Dienes } & \text { venous } & 137.3 \pm 9.2 & 136.9 \pm 8.8 & -0.35 \pm 2.50 \\ \text { ( } \mu \mathrm{mol} / \mathrm{mmol} \text { LDL-C) } & \text { arterial } & 137.4 \pm 8.1 & 137.0 \pm 7.6 & -0.42 \pm 4.05 \\ \text { Rate } & & & & \\ \text { (dienes/min) } & \text { venous } & 3.81 \pm 0.63 & 3.54 \pm 0.47 & -0.28 \pm 0.29 \dagger \\ & \text { arterial } & 3.88 \pm 0.53 & 3.57 \pm 0.54 & -0.31 \pm 0.22 \dagger\end{array}$

Just before PTCA, venous (V1) and arterial (A1) blood samples were taken, and analyzed as described in 'Methods'. Ten minutes after the first balloon inflation, again a venous (V2) and arterial (A2) sample were taken, and the changes $(\Delta \mathrm{V}, \Delta \mathrm{A})$ as compared with respectively $\mathrm{V} 1$ and $\mathrm{A} 1$ were calculated. Values are means \pm SD. ${ }^{*} n=12$ for LDL lipid hydroperoxides. Short-term changes were analyzed by signed rank test, $\dagger: P<0.002$. No significant differences between venous and arterial samples were noticed. $L D L-C=$ LDL cholesterol. 
Just prior to PTCA, the mean plasma lipid hydroperoxide level was $0.24 \pm 0.17$ $\mu \mathrm{mol} / \mathrm{mol}$ cholesterol in venous samples, and $0.32 \pm 0.52 \mu \mathrm{mol} / \mathrm{mol}$ in arterial samples. Ten minutes after the first balloon inflation, levels remained essentially similar, both in venous and arterial plasma (Table 6.1). Lipid hydroperoxide levels in LDL also showed no effect of ballooning (Table 6.1). However, levels in LDL were much higher (irrespective of standardization for cholesterol), and did not correlate with levels in plasma at any point of sampling $(r=-0.32$ to $+0.51, p \geq 0.09, n=9-12)$.

\section{$L D L$ oxidation in vitro}

The lagtime before onset of rapid oxidation ${ }^{21}$, and the amount of dienes formed during copper-catalyzed oxidation of LDL in vitro, were similar just before PTCA and 10 minutes after the first balloon inflation, in both venous and arterial samples (Table 6.1). During this period, however, the maximum rate of diene formation had decreased by $0.28 \pm 0.29 \mu \mathrm{mol} / \mathrm{mmol} \mathrm{LDL}$ cholesterol $/ \mathrm{min}$ or $6.7 \pm 6.2 \%(P=0.002)$ in venous samples, and by $0.31 \pm 0.22 \mu \mathrm{mol} / \mathrm{mmol} / \mathrm{min}$ or $7.9 \pm 5.1 \%(P=0.0002)$ in arterial samples (Table 6.1). The maximum rate of oxidation did not correlate with antioxidant levels. Although there was a significant correlation between the maximum rate of oxidation and the (preformed) lipid hydroperoxide levels in the original LDL solution obtained before PTCA (V1: $r=0.70, P=0.017$; $A 1$ : $r=0.87, P=0.0005$ ), this correlation was not found between the short-term changes of both parameters.

\section{$L D L$ antioxidants}

During the ten minutes after the first balloon infiation, cholesterol-standardized $\alpha$-tocopherol levels in LDL increased significantly in arterial samples by $192.2 \pm 291.5$ $\mathrm{nmol} / \mathrm{mmol}$ cholesterol or $6.3 \pm 9.4 \%(P=0.033)$, but not in venous samples (Table 6.2). A similar trend was seen for $\beta+\gamma$-tocopherol (arterial increase: $15.2 \pm 27.0$ $\mathrm{nmol} / \mathrm{mol}$ or $6.2 \pm 10.2 \%, P=0.068$ ). Arterial $\delta$-tocopherol increased non-significantly by $1.0 \pm 2.2 \mathrm{nmol} / \mathrm{mol}$ or $6.8 \pm 18.7 \%(P=0.146)$.

Cholesterol-standardized total carotenoid levels (without phytofluene) in LDL decreased in venous samples $(5.8 \pm 10.1 \mathrm{nmol} / \mathrm{mmol}$ cholesterol or $2.9 \pm 4.4 \%, P=$ $0.040)$, but only the small change in the $B$-carotene level $(-1.8 \pm 3.4 \mathrm{nmol} / \mathrm{mmol}$ cholesterol or $-2.7 \pm 3.8 \%, P=0.033$ ) reached statistical significance. The decreases in venous $B$-cryptoxanthin $(1.0 \pm 1.9 \mathrm{nmol} / \mathrm{mmol}$ cholesterol, $\mathrm{P}=0.057)$ and lycopene $(1.6 \pm 2.7 \mathrm{nmol} / \mathrm{mmol}$ cholesterol, $\mathrm{P}=0.057)$ levels were of borderline significance. As 
with tocopherol levels, all carotenoid levels in arterial samples increased, but these increases did not reach statistical significance. The increase in arterial $\alpha$-carotene $(1.1 \pm 2.8 \mathrm{nmol} / \mathrm{mmol}$ cholesterol) levels was of borderline significance $(P=0.057)$. For most carotenoids, the short-term responses in venous samples were in opposite direction from those in arterial samples (Table 6.2). These differences in change reach statistical significance for all carotenoids except for lutein + canthaxanthin.

Table 6.2. Short-term effect of PTCA on cholesterol-standardized LDL antioxidant levels in venous and arterial samples.

\begin{tabular}{|c|c|c|c|c|}
\hline & Sample & $\begin{array}{r}V 1 \text { or } A 1 \\
(n=13)\end{array}$ & $\begin{array}{r}\text { V2 or A2 } \\
(n=13)\end{array}$ & $\begin{array}{l}\text { Change } \\
(\Delta V, \Delta A)\end{array}$ \\
\hline \multicolumn{5}{|c|}{ Cholesterol-standardized LDL antioxidants ( $\mathrm{nmol} / \mathrm{mmol}$ cholesterol) } \\
\hline \multirow[t]{2}{*}{ Total tocopherols ${ }^{a}$} & venous & $3865 \pm 598$ & $3836 \pm 641$ & $-28.6 \pm 173.1$ \\
\hline & arterial & $3755 \pm 682$ & $3963 \pm 640$ * & $208.4 \pm 316.3+$ \\
\hline$\delta$-Tocopherol & $\begin{array}{l}\text { venous } \\
\text { arterial }\end{array}$ & $\begin{array}{l}20 \pm 8 \\
20 \pm 8\end{array}$ & $\begin{array}{l}21 \pm 8 \\
21 \pm 8\end{array}$ & $\begin{array}{l}0.3 \pm 1.7 \\
1.0 \pm 2.2\end{array}$ \\
\hline B+ $\gamma$-Tocopherol & $\begin{array}{l}\text { venous } \\
\text { arterial }\end{array}$ & $\begin{array}{l}365 \pm 224 \\
358 \pm 231\end{array}$ & $\begin{array}{l}363 \pm 223 \\
373 \pm 230\end{array}$ & $\begin{array}{l}-2.1 \pm 21.0 \\
15.2 \pm 27.0\end{array}$ \\
\hline \multirow[t]{2}{*}{$\alpha$-Tocopherol } & venous & $3480 \pm 527$ & $3453 \pm 571$ & $-26.8 \pm 152.6$ \\
\hline & arterial & $3376 \pm 579$ & $3569 \pm 579 *$ & $192.2 \pm 291.5 \dagger$ \\
\hline \multirow[t]{2}{*}{ Total carotenoids ${ }^{a}$} & venous & $247 \pm 119$ & $241 \pm 120$ & $-5.8 \pm 10.1 \dagger$ \\
\hline & arterial & $238 \pm 111$ & $249 \pm 127$ & $11.1 \pm 26.3$ \\
\hline \multirow[t]{2}{*}{ Lutein ${ }^{\mathbf{b}}$} & venous & $40 \pm 10$ & $39 \pm 10$ & $-1.0 \pm 2.1$ \\
\hline & arterial & $39 \pm 9$ & $40 \pm 9^{\star}$ & $0.8 \pm 3.5$ \\
\hline \multirow[t]{2}{*}{ B-Cryptoxanthin } & venous & $30 \pm 13$ & $29 \pm 14$ & $-1.0 \pm 1.9$ \\
\hline & arterial & $29 \pm 13$ & $30 \pm 14$ & $1.2 \pm 4.1$ \\
\hline \multirow[t]{2}{*}{ Lycopene } & venous & $75 \pm 43$ & $73 \pm 43$ & $-1.6 \pm 2.7$ \\
\hline & arterial & $72 \pm 41$ & $75 \pm 43$ & $2.9 \pm 7.2$ \\
\hline \multirow[t]{2}{*}{$\alpha$-Carotene } & venous & $18 \pm 16$ & $17 \pm 16$ & $-0.4 \pm 1.0$ \\
\hline & arterial & $17 \pm 16$ & $18 \pm 18$ * & $1.1 \pm 2.8$ \\
\hline \multirow[t]{2}{*}{ B-Carotene } & venous & $85 \pm 62$ & $83 \pm 62$ & $-1.8 \pm 3.4 \dagger$ \\
\hline & arterial & $81 \pm 59$ & $86 \pm 67$ * & $5.0 \pm 11.7$ \\
\hline \multirow[t]{2}{*}{ Phytofluene $^{c}$} & venous & $98 \pm 95$ & $98 \pm 96$ & $0.0 \pm 4.4$ \\
\hline & arterial & $93 \pm 92$ & $101 \pm 106$ & $7.9 \pm 16.9$ \\
\hline
\end{tabular}

$a$ a, sum of specified tocopherols or carotenoids (phytofluene not included); b. lutein levels may include small amounts of canthaxanthin; $c$, phytofluene levels are presented in peak area/ $\mu \mathrm{mol}$ LDL cholesterol. Values are means \pm SD.

$t$, short-term changes were analyzed by signed rank test: $P<0.05$.

${ }^{\star}$ and ${ }^{\star *}$, significant differences between venous and arterial values, ${ }^{*}, P<0.05 ;{ }^{* *}, P<0.01$. 


\section{Longer-term effects of PTCA}

On the second day after PTCA (V3), lipid hydroperoxide levels and LDL oxidation parameters were similar to values at the start of the study (VO) (Table 6.3). Most cholesterol-standardized antioxidant levels in LDL were also comparable to levels at Vo, except that $\delta$-tocopherol and $B+\gamma$-tocopherol levels had decreased by $4.5 \pm 5.0$ $\mathrm{nmol} / \mathrm{mmol}$ cholesterol $(16.4 \pm 20.9 \%, P=0.037)$ and $96.2 \pm 109.7 \mathrm{nmol} / \mathrm{mmol}$ cholesterol ( $17.5 \pm 23.1 \%, \mathrm{P}=0.014$ ), respectively (Table 6.3).

Table 6.3. Longer-term effect of PTCA on plasma and LDL lipid hydroperoxide levels, LDL oxidation in vitro, and LDL antioxidant levels.

\begin{tabular}{|c|c|c|c|c|}
\hline & Vo $\left(n=10^{a}\right)$ & {$[n=13]$} & V3 $\left(n=10^{a}\right)$ & Change $(\Delta \mathrm{V})$ \\
\hline \multicolumn{5}{|c|}{ Plasma and LDL lipid hydroperoxides } \\
\hline Plasma & $0.16 \pm 0.10$ & [0.17] & $0.15 \pm 0.08$ & $-0.01 \pm 0.07$ \\
\hline LDL & $1.03 \pm 0.31$ & [1.05] & $1.12 \pm 0.38$ & $0.09 \pm 0.43$ \\
\hline \multicolumn{5}{|l|}{$L D L$ oxidation } \\
\hline Lagtime & $62.3 \pm 13.2$ & [63.5] & $62.5 \pm 13.0$ & $0.20 \pm 6.05$ \\
\hline Dienes & $137.0 \pm 10.3$ & [138.0] & $136.5 \pm 9.1$ & $-0.56 \pm 4.50$ \\
\hline Rate & $3.16 \pm 0.46$ & [3.35] & $3.21 \pm 0.36$ & $0.05 \pm 0.32$ \\
\hline \multicolumn{5}{|l|}{ LDL antioxidants } \\
\hline Total tocopherols ${ }^{b}$ & $3882 \pm 654$ & [3798] & $3757 \pm 681$ & $-125.4 \pm 317.2$ \\
\hline$\delta$-Tocopherol & $21 \pm 7$ & [20] & $16 \pm 4$ & $-4.5 \pm 5.0 \dagger$ \\
\hline$B+\gamma$-Tocopherol & $367 \pm 226$ & [363] & $271 \pm 126$ & $-96.2 \pm 109.7 \dagger$ \\
\hline$\alpha$-Tocopherol & $3495 \pm 557$ & [3414] & $3470 \pm 634$ & $-25.1 \pm 279.6$ \\
\hline Total carotenoids ${ }^{b}$ & $262 \pm 133$ & [250] & $264 \pm 130$ & $1.6 \pm 18.6$ \\
\hline Lutein ${ }^{b}$ & $41 \pm 11$ & [40] & $40 \pm 10$ & $-1.4 \pm \quad 3.9$ \\
\hline B-Cryptoxanthin & $31 \pm 17$ & [30] & $32 \pm 16$ & $0.5 \pm 2.4$ \\
\hline Lycopene & $81 \pm 42$ & [76] & $81 \pm 41$ & $0.4 \pm 7.5$ \\
\hline$\alpha$-Carotene & $19 \pm 18$ & [18] & $18 \pm 18$ & $-0.6 \pm 0.6$ \\
\hline B-Carotene & $90 \pm 72$ & [87] & $93 \pm 70$ & $2.4 \pm 13.6$ \\
\hline Phytofluene $^{b}$ & $106 \pm 104$ & [99] & $108 \pm 105$ & $1.7 \pm 10.6$ \\
\hline
\end{tabular}

On the moming of PTCA (V0) and two days thereafter (V3) a venous blood sample was taken, and the changes $(\Delta V)$ in lipid hydroperoxide levels, LDL oxidation and antioxidant levels were calculated (for units, see Table 6.1 and 6.2). Values are means \pm SD. Values in brackets are means of all 13 LDL samples.

a $n=9$ [12] for LDL lipid hydroperoxides, and $n=9$ [13] for LDL oxidation parameters

bee superscripts a-c in Table 6.2.

$t$, longer-term changes were analyzed by signed rank test, $P<0.05$. 


\section{Comparison between venous and arterial samples}

Spearman correlation coefficients between venous (V1) and arterial (A1) samples taken just before PTCA were 0.76 for plasma lipid hydroperoxides $(P=0.003, n=13)$, and 0.55 for lipid hydroperoxides in $L D L(P=0.067, n=12)$. For $L D L$ oxidation parameters, Spearman correlations ranged from 0.86 to $0.93(P \leq 0.0001, n=13$ ), and for LDL antioxidants from 0.90 to 1.00 ( $P \leq 0.0001, n=13$ ). Before PTCA, there were also no significant differences between the mean venous and arterial levels for any of the parameters studied (Tables 6.1 and 6.2).

\section{DISCUSSION}

Several studies have shown the formation of free radicals in animal models for ischemia and reperfusion ${ }^{1-3}$, and after PTCA in humans ${ }^{13,14}$. Formation of lipid hydroperoxides after PTCA due to free radical attack of polyunsaturated fatty acids, however, could not be demonstrated in the present study. No increase in plasma lipid hydroperoxide levels was noticed ten minutes after the first balloon inflation, nor 2 days thereafter.

The absence of increased levels of lipid hydroperoxides after PTCA is supported by measurements of TBArs in coronary sinus plasma, two and ten minutes after the last balloon inflation ${ }^{18}$. Other studies ${ }^{15,16}$, however, have suggested a transient release of TBArs in coronary venous plasma immediately after PTCA, but this was not confirmed by Steg et al. ${ }^{19}$. A release of TBArs, only detectable during the first minute after PTCA, is further not supported by Blann et al. ${ }^{28}$, who found no increased levels in coronary artery plasma at the time of balloon inflation, but levels were increased after 10 minutes. These longer-lasting effects are in agreement with the reported presence of free radicals in coronary sinus plasma for at least 10 minutes following balloon inflations ${ }^{13}$. However, not all increases in TBArs reflect increased lipid peroxidation ${ }^{17}$.

In the present study no increases in lipid hydroperoxides in LDL following PTCA were noticed either. Levels were lower than those reported by El-Saadani et al. ${ }^{29}$, who used an iodometric method to detect lipid hydroperoxides. They reported mean 
lipid hydroperoxide levels in native LDL of $5.4 \pm 0.3 \mathrm{nmol} / \mathrm{mg}$ LDL (approx. 6 $\mu \mathrm{mol} / \mathrm{mmol} \mathrm{LDL}$ cholesterol). In the present study, however, the amount of lipid hydroperoxides in LDL was higher than the amount that could be detected in plasma, and most lipid hydroperoxides in LDL were, therefore, probably formed during the isolation of LDL, despite the protective presence of EDTA. This may also explain the lack of correlation between lipid hydroperoxide levels in plasma and LDL.

To investigate whether free radicals formed during or after PTCA had modified LDLs, copper-catalyzed oxidation of isolated LDL in vitro and LDL antioxidant concentrations were investigated. The lagtime and the maximum amount of dienes during oxidation of LDL in vitro, were not affected by PTCA. The unchanged lagtimes indicate that the oxidation resistance of LDL was not affected, despite the changes in antioxidant levels during the first ten minutes of PTCA. The remarkable longer-term decreases in $\delta$ - and $B+\gamma$-tocopherol also were not associated with diminished lagtimes.

Recently, it has been reported that the copper-catalyzed LDL oxidizability was reduced in patients with coronary artery disease taking the B-blockers Atenolol or Metoprolol, as compared with patients not taking any B-blockers ${ }^{30}$. LDL oxidizability was not affected by intake of acetylsalicylic acid, nitrates or calcium antagonists. In the present study, however, average lipid peroxide levels and LDL oxidation parameters, as well as responses during the study, were similar in the 6 patients receiving B-blockers (1 patient used Atenolol, 4 patients Metoprolol, and 1 patient Bisoprolol) and the 7 patients not taking B-blockers (results not shown). It is, therefore, unlikely that the intake of B-blockers has prevented detection of PTCA-induced lipid peroxidation.

The maximum rate of oxidation decreased by $7-8 \%$ during the ten minutes after the first balloon inflation, an effect observed in both venous and arterial samples. Although it is apparent that the lagtime and amount of dienes reflect the LDL antioxidant and PUFA concentration, factors affecting the maximum rate of oxidation are unclear. Cominacini et $a l_{.}^{31}$, reported that the maximum rate of oxidation decreased after $\alpha$-tocopherol supplementation. It is unlikely, however, that changes in LDL tocopherol or carotenoid levels affected the maximum rate of oxidation in this study, since antioxidant responses in venous and arterial samples were opposite, whereas the maximum rate of oxidation decreased in both. $A$ change in (preformed) lipid hydroperoxide levels in LDL could also not explain the decreased maximum rate of oxidation, since there was no correlation between the short-term responses of these parameters. Surprisingly, the maximum rate of oxidation just before PTCA (V1: 3.81 
dienes/min) was higher than the rate at baseline (V0: 3.35 dienes/min), indicating an effect on the rate of oxidation not related to the ischemia or reperfusion during angioplasty (Figure 6.2). Although it is possible that factors like the administered heparin or lidocaine may have caused this initial increase in maximum rate of oxidation, it is inconceivable that the effect of these factors diminished suddenly during the short period of 10 minutes between V1, A1 and V2, A2. LDL from patients receiving nitrates demonstrated similar copper-catalyzed oxidizability as compared with LDL from patients not taking nitrates ${ }^{30}$, but the effects of nitroglycerine and contrast fluid, administered i.c. after $\mathrm{V} 1$ and $\mathrm{A} 1$, on the maximum rate of oxidation is unknown.

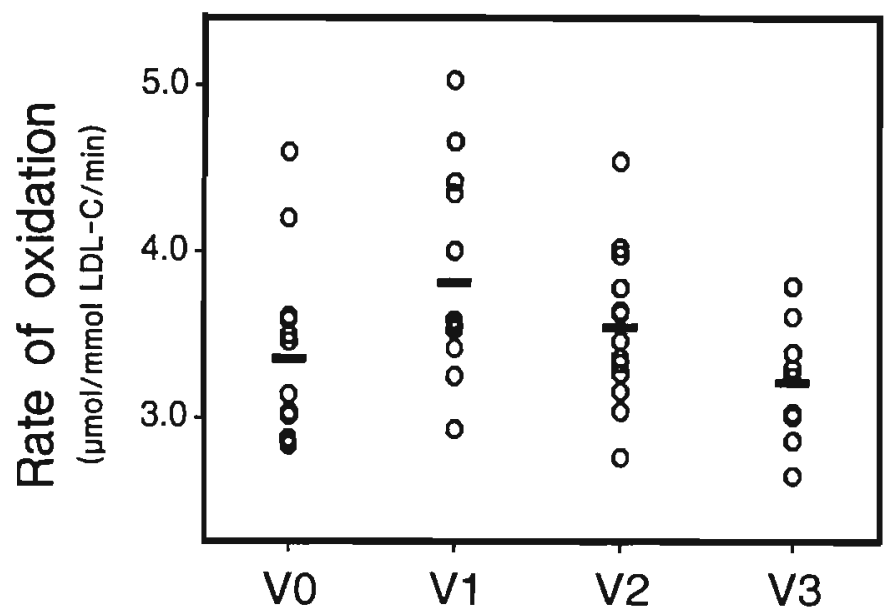

Figure 6.2. Maximum rate of in vitro oxidation of LDL from venous samples (for further explanation see methods and Figure 6.1). Circles indicate individual values, and horizontal bars indicate mean values. $L D L-C=L D L$ cholesterol.

Apart from the change in maximum rate of oxidation of LDL in vitro, LDL carotenoid levels decreased slightly in venous samples during the first ten minutes of PTCA, and, unexpectedly, LDL antioxidant levels increased in arterial samples. This latter observation does not agree with other studies, which showed decreased $\alpha$ tocopherol levels in heart tissue $e^{32,33}$ and pulmonary artery plasma ${ }^{34}$ after other procedures associated with ischemia and reperfusion. However, the periods of 
ischemia in these studies were much longer than those used during PTCA in the present study, and results may therefore not be comparable. The origin of the extra antioxidants in LDL of arterial samples after ballooning in the present study is unknown, however. In theory, it is possible that the transfer of $\alpha$-tocopherol from LDL to cells is inhibited due to heparin-induced release of lipoprotein lipase (LPL) from the cells ${ }^{35}$, thereby causing a retainment of $\alpha$-tocopherol in LDL. However, the comparable decreases in plasma triglycerides levels in venous and arterial samples (data not shown) indicate that the heparin-induced release of LPL was similar in venous and arterial blood, whereas the $\alpha$-tocopherol responses were not. Although LDL $\delta$ - and $\beta+\gamma$-tocopherol levels are low as compared with the large pool of $\alpha$ tocopherol, the decrease of almost $20 \%$ two days after PTCA indicates a selective loss of these isomers. We cannot rule out, however, that the standard hospital meals may have contained little $\delta$ - and $\beta+\gamma$-tocopherol.

In summary, we found no clear evidence for increased lipid peroxidation 10 minutes after the first balloon inflation during PTCA as measured by lipid hydroperoxides levels, and the lagtime and maximum amount of dienes formed during oxidation of LDL in vitro. There was a significant decrease of carotenoid levels in venous LDL samples, but it was very small. Furthermore, the decrease in maximum rate of diene formation during oxidation of LDL in vitro and the increased coronary arterial levels of especially LDL- $\alpha$-tocopherol rather indicate decreased lipid peroxidation. It is unclear whether the almost $20 \%$ decrease in LDL $\delta$ - and $B+\gamma$ tocopherol levels in venous samples two days after PTCA has any biological significance.

In conclusion, our results do not support that PTCA in humans is associated with oxidative stress-induced lipid peroxidation.

\section{Acknowledgments}

We are indebted to the health professionals of the Department of Cardiology of the Academic Hospital Maastricht whose cooperation made this study possible, and to Mrs Carin Wensing who assisted in collecting blood samples. 


\section{REFERENCES}

1. Zweier JL, Kuppusamy P, Williams R, Raybum BK, Smith D, Weisfeldt ML, Flaherty JT. Measurement and characterization of postischemic free radical generation in the isolated perfused heart. J Biol Chem 1989; 264:18890-18895.

2. Tosaki A, Blasig IE, Pali T, Ebert B. Heart protection and radical trapping by DMPO during reperfusion in isolated working rat hearts. Free Rad Biol Med 1990; B:363-372.

3. Bolli R, Patel BS, Jeroudi MO, Lai EK, MCCay PB. Demonstration of free radical generation in "stunned" myocardium of intact dogs with the use of the spin trap alpha-phenyl $\mathrm{N}$-tert-butyl nitrone. $\mathrm{J}$ Clin Invest 1988; 82:476-485.

4. Gardner HW. Oxygen radical chemistry of polyunsaturated fatty acids. Free Rad Biol Med 1989; 7:65-86.

5. Halliwell B, Gutteridge JMC. Role of free radicals and catalytic metal ions in human disease: An overview. Meth Enzymol 1990; 186:1-85.

6. Rice-Evans $C$, Burdon R. Free radical-lipid interactions and their pathological consequences. Prog Lipid Res 1993; 32:71-110.

7. Myers ML, Bolli R, Lekich RF, Hartley CJ, Roberts R. Enhancement of recovery of myocardial function by oxygen free-radical scavengers after reversible regional ischemia. Circulation 1985; 72:915-921.

8. Opie LH. Reperfusion injury and its pharmacologic modification. Circulation 1989; 80:1049-1062.

9. Ambrosio G, Flaherty JT, Duilio C, Tritto I, Santoro G, Elia PP, Condorelli M, Chiariello M. Oxygen radicals generated at reflow induce peroxidation of membrane lipids in reperfused hearts. $J$ Clin Invest 1991; 87:2056-2066.

10. Barrington PL, Lai E, McCay PB. Lack of myocardial lipid peroxidation during acute reperfusion injury in perfused guinea pig hearts. Cardiovasc Res 1993; 27:1339-1345.

11. Rabl H, Khoschsonur G, Colombo T, Tatzber F, Esterbauer $H$. Human plasma lipid peroxide levels show a strong transient increase after successful revascularization operations. Free Rad Biol Med 1992; 13:281-288.

12. Tihan $T$, Chiba $P$, Krupicka $O$, Fritzer $M$, Seitelberger $R$, Müller MM. Serum lipid peroxide levels in the course of coronary by-pass surgery. Eur J Clin Chem Clin Biochem 1992; 30:205-208.

13. Coghlan JG, Flitters WD, Holley AE, Norell M, Mitchell AG, Ilsley CD, Slaters TF. Detection of free radicals and cholesterol hydroperoxides in blood taken from the coronary sinus of man during percutaneous transluminal coronary angioplasty. Free Rad Res Commun 1991; 14:409-417.

14. Grech ED, Dodd NJF, Bellamy CM, Perry RA, Morrison WL, Ramsdale DR. Free-radical generation during angioplasty reperfusion for acute myocardial infarction. Lancet 1993; 341:990-991.

15. Roberts MJD, Young IS, Trouton TG, Trimble ER, Khan MM, Webb SW, Wilson CM, Patterson GC, Adgey AAJ. Transient release of lipid peroxides after coronary artery balloon angioplasty. Lancet 1990; 336:143-145.

16. Oldroyd KG, Paterson JR, Rumley AG, Eteiba H, Rae AP, Shepherd J, Cobbe SM, Hutton I. Coronary venous lipid peroxide concentrations after coronary angioplasty: correlation with biochemical and electrocardiographic evidence of myocardial ischaemia. Br Heart $\mathrm{J}$ 1992; 68:43-47.

17. Janero DR. Malondialdehyde and thiobarbituric acid-reactivity as diagnostic indices of lipid peroxidation and peroxidative tissue injury. Free Rad Biol Med 1990; 9:515-540. 
18. Paterson JR, Oldroyd KG, Rumley AG, Eteiba H, Rae AP, Hutton I, Cobbe SM. Free radical activity during percutaneous trans-luminal coronary angioplasty. Biochem Soc Trans 1990; 18:1183-1184.

19. Steg PG, Pasquier C, Pham T, Martin S, Juliard JM, Himbert D, Pociadalo MA, Gourgon R, Hakim J. Evidence for neutrophil and complement activation early after PTCA. J Am Coll Cardiol 1990; 15:164A (abstract).

20. Terpstra AHM, Woodward CJH, Sanchez-Muniz FJ. Improved techniques for the separation of serum lipoproteins by density gradient ultracentrifugation: Visualization by prestaining and rapid separation of serum lipoproteins from small volumes of serum. Anal Biochem 1981; 111:149-157.

21. Esterbauer $\mathrm{H}$, Striegl G, Puhl $\mathrm{H}$, Rotheneder $\mathrm{M}$. Continuous monitoring of in vitro oxidation of human low density lipoprotein. Free Rad Res Commun 1989; 6:67-75.

22. Hess D, Keller HE, Oberlin B, Bonfanti R, Schūep W. Simultaneous determination of retinol, tocopherols, carotenes and lycopene in plasma by means of high-performance liquid chromatography on reversed phase. Int J Vitam Nutr Res 1991; 61:232-238.

23. Schudel P, Mayer H, Isler O. Tocopherols. II. Chemistry. In: Sebrell WH jr, Harris RS, eds. The Vitamins. Vol. 5. New York: Academic Press, 1972:168-218.

24. The Merck Index. 11th ed. Rahway, N.J.: Merck \& Co., 1989:884,1495,1579.

25. Nierenberg DW, Nann SL. A method for determining concentrations of retinol, tocopherol, and five carotenoids in human plasma and tissue samples. Am J Clin Nutr 1992; 56:417-426.

26. Handelman GJ, Machlin LJ, Fitch K, Weiter JJ, Dratz EA. Oral $\alpha$-tocopherol supplements decrease plasma $\gamma$-tocopherol levels in humans. J Nutr 1985; 115:807-813.

27. De Leenheer AP, De Bevere VORC, Claeys AE. Measurement of $\alpha$-, $\beta$-, and $\gamma$-tocopherol in serum by liquid chromatography. Clin Chem 1979; 25:425-428.

28. Blann A, Midgley $H$, Burrows $G$, Maxwell $S$, Utting $S$, Davies $M$, Waite $M$, McCollum $C$. Free radicals, antioxidants, and endothelial cell damage after percutaneous transluminal coronary angioplasty. Cor Art Dis 1993; 4:905-910.

29. El-Saadani M, Esterbauer H, El-Sayed M, Goher M, Nassar AY, Jūrgens GA. spectrophotometric assay for lipid peroxides in serum lipoproteins using a commercially available reagent. J Lipid Res 1989; 30:627-630.

30. Croft KD, Dimmitt SB, Moulton C, Beilin LJ. Low density lipoprotein composition and oxidizability in coronary disease; apparent favourable effect of beta blockers. Atherosclerosis 1992; 97:123-130.

31. Cominacini L, Garbin U, Cenci B, Davoli A, Pasini C, Ratti E, Gaviraghi G, Lo Cascio V, Pastorino AM. Predisposition to $L D L$ oxidation during copper-catalyzed oxidative modification and its relation to $\alpha$-tocopherol content in humans. Clin Chim Acta 1991; 204:57-68.

32. Barsacchi R, Pelosi G, Maffei S, Baroni M, Salvatore L, Ursini F, Verunelli F, Biagini A. Myocardial vitamin $\mathrm{E}$ is consumed during cardiopulmonary bypass: indirect evidence of free radical generation in human ischemic heart. Int J Cardiol 1992; 37:339-343.

33. Weisel RD, Mickle DAG, Finkle CD, Tumiati LC, Madonik MM, Ivanov J, Burton GW, Ingold KU. Myocardial free-radical injury after cardioplegia. Circulation 1989; 80(suppl III):III-14-III-18.

34. Murphy ME, Kolvenbach R, Aleksis M, Hansen R, Sies $H$. Antioxidant depletion in aortic crossclamping ischemia: Increase of the plasma $\alpha$-tocopheryl quinone/ $\alpha$-tocopherol ratio. Free Rad Biol Med 1992; 13:95-100.

35. Kayden HJ, Traber MG. Absorption, lipoprotein transport, and regulation of plasma concentrations of vitamin $E$ in humans. J Lipid Res 1993; 34:343-358. 


\section{GENERAL DISCUSSION}

The aim of the studies described in this thesis was to investigate parameters of lipid peroxidation and antioxidant status in situations in which the degree of oxidative stress and/or antioxidative protection is altered. Firstly, we have increased the availability of a major substrate for lipid peroxidation, polyunsaturated fatty acids (PUFA), by supplementing the diet with highly unsaturated fish oil concentrates. At the same time we have studied whether increasing the dietary intake of vitamin $E$, a chainbreaking antioxidant, would be necessary to compensate for the increased availability of substrate. In a second study this dietary manipulation was combined with exercise, during which increased production of oxygen free radicals may exacerbate lipid peroxidation. Thereafter, the antioxidant status was studied in pregnant women. During the course of pregnancy, plasma (polyunsaturated) fatty acids levels increase. The increased availability of substrate for lipid peroxidation may compromise antioxidant defences. The antioxidant status was also studied in women with pregnancy-induced hypertension (PIH), since it has been suggested that many of the clinical and pathophysiological features of PIH might be explained by an inadequate antioxidant status. Finally, lipid peroxidation and antioxidant status were studied during revascularization of obstructed coronary arteries, a medical procedure associated with increased production of oxygen free radicals.

The major parameters studied were oxidation of low density lipoproteins (LDL) in vitro and lipid soluble antioxidant concentrations in plasma. In subjects studied during exercise, also various parameters linked to exercise or exercise performance were examined. 


\section{MAIN FINDINGS}

\section{Oxidation of $L D L$ in vitro}

\section{Fish oil supplementation}

In the first study (Chapter 2), fish oil concentrates, with or without vitamin $E$, were given to sedentary men for 3 weeks, and oxidation of LDL in vitro was compared with a control group receiving no supplement. Already after the first week, an increase in the in vitro formation of conjugated dienes during oxidation of LDL (oxidizability) was noticed in both groups receiving fish oil, as compared with controls. After three weeks, these increases were significantly different from controls. These results support the hypotheses that an increase in the average number of double bonds in fatty acids in the diet increases the in vitro oxidizability of LDL, as was also found when the proportion of dietary n-6 PUFA was increased at the expense of monounsaturated fatty acids ${ }^{1,2,3,4}$. Furthermore, our results agree with studies showing an increase in the formation of thiobarbituric acid reactive substance during cell- or copper-catalyzed oxidation of LDL in vitro ${ }^{5,6.7}$ after fish oil supplementation. However, in the second study (Chapter 3 ), fish oil supplementation did not increase the oxidizability of LDL.

In both our studies a parallel design was used, and a control group was present to correct for unknown drifts with time. Furthermore, samples from each of the three experimental groups were present in each analytical run, and between-run artefacts were therefore excluded. However, three apparent differences existed between the two studies: (1) subjects were sedentary in the first study, and well-trained in the second study, (2) atthough fish oil concentrates used in both studies were derived from natural sources and gelatin covered, they were supplied by different manufacturers, (3) and LDL for oxidation experiments were prepared by different methods.

With respect to the first two differences, both fish oil supplements increased the degree of fatty acid unsaturation in the sedentary as well as the trained subjects. In the sedentary subjects receiving fish oil (Chapter 2), the proportion of n-3 fatty acids in plasma phospholipids increased from $4.7 \%$ to $9.7 \%$. In the trained subjects (Chapter 3), n-3 fatty acids in red blood cell phospholipids increased from $5.2 \%$ to $7.3 \%$, slightly less than half the increase observed in plasma phospholipids in the first study. However, it is known that n-3 fatty acid responses in red blood cells are less 
pronounced than responses in plasma $a^{5,8,9}$. Since dietary supplementations of $n-3$ fatty acids were comparable in both studies (study 1: $2.4 \mathrm{~g} /$ day; study 2: $2.1 \mathrm{~g} /$ day), we expect that the LDL fatty acid composition also changed similarty. Unfortunately, fatty acid compositions of LDL were not analyzed, but based on data from others studies $^{7,10}$ increases in the proportion of $n-3$ fatty acids in LDL of $3-5 \%$ might be expected. Comparison of the two methods of LDL preparation (difference no. 3) revealed no significant differences in LDL oxidation parameters in samples from not supplemented sedentary subjects (results not shown). To investigate whether fish oil supplementation may have had different effects on the two methods, a second study was carried out in which 6 male sedentary subjects received Fish-EPA capsules (6 g/day, Orthica, Weesp, The Netherlands) and 6 received placebo capsules, for 3 weeks (Plat et al., results not yet published). The fish oil capsules were the same as used in the second study (Chapter 3 ). For both methods, fish oil supplementation did not increase the maximum amount of dienes, consistent with results shown in Chapter 3. In the first study (Chapter 2), the number of subjects was limited. Nonetheless, the amount of dienes was consistently increased after 1, 2, and 3 weeks of supplementation. Furthermore, dienes were increased by approximately $20 \%$ in both groups receiving fish oil supplementation. Although currently we cannot explain why, the discrepancy between the results reported in Chapter 2 and 3 may be related to the kind of fish oil capsules. Since the fish oil capsules used in the first study were not available anymore, we have not been able to verify this however.

In theory, an increase in fatty acid unsaturation of LDL, and thus of substrate for lipid peroxidation, may decrease the lagtime before rapid onset of oxidation in vitro due to accelerated depletion of antioxidants, and may increase the rate of oxidation due to an increased number of lipid peroxidation chain-reactions. Indeed, in the first study (Chapter 2), fish oil supplementation tended to decrease the lagtime in agreement with recent studies in elderly hypertensive subjects ${ }^{7}$ and postmenopausal women ${ }^{11}$, but this was not confirmed in our second study (Chapter 3). Similar inconsistent result were also found when the effects of dietary linoleic acid-rich diets were compared with oleic acid-rich diets ${ }^{2,4}$.

In the first study, fish oil supplementation did not significantly affect the rate of oxidation. In contrast with our expectations, however, fish oil supplementation 
decreased the rate of oxidation in the second study, especially in combination with vitamin $E$ supplementation. A decrease in rate of oxidation after fish oil supplementation was recently also found in elderly hypertensive subjects ${ }^{7}$ and postmenopausal women ${ }^{11}$. This appears to be in disagreement with studies in which a linoleic acid-rich diet increased the rate of oxidation, as compared with an oleic acidrich diet ${ }^{2,4}$. Recently, Kleinveld et al. ${ }^{12}$ found that the rate of oxidation standardized for LDL protein, correlated positively with the proportion of linoleic acid, and inversely with the proportion of oleic acid and the oleic acid/linoleic acid ratio. However, correlations with n-3 fatty acids were not studied. Furthermore, phospholipid fatty acid analysis in plasma (Chapter 2) and erythrocytes (Chapter 3 ) indicated that fish oil supplementation decreased the proportion of linoleic acid and increased the oleic acid/linoleic acid ratio in both our studies, whereas a decrease in rate of oxidation was only noticed in the second one. In other fish oil supplementation studies, the rate of oxidation decreased while the oleic acid/linoleic acid ratio in $\mathrm{LDL}^{7}$ and plasma ${ }^{11}$ did not increase. The underlying mechanism for the possible effect of fish oil supplementation on the rate of oxidation remains unexplained.

In conclusion, the first study indicated that fish oil supplementation may promote oxidation of LDL, which is further supported by studies showing an increase in the uptake of native ${ }^{5}$ and copper-oxidized ${ }^{7}$ LDL by macrophages after fish oil supplementation. However, a reducing effect on the rate of copper-catalyzed oxidation of LDL in vitro was found in our second study. These contradictory results, and the inconsistencies and apparent paradoxes reported by others, indicate that further studies are needed before definite conclusions can be drawn about the effect of fish oil supplementation on oxidation of LDL in vitro.

\section{Fish oil plus vitamin E supplementation}

In both studies described in Chapter 2 and 3, supplementation with 300 IU of vitamin $E$, additionally to fish oil supplementation, almost doubled vitamin $E$ levels in LDL, and increased the lagtime, most likely by acting as a chain-breaking antioxidant which terminates lipid peroxidation chain reactions until complete depletion of the antioxidant(s) ${ }^{13}$. It should be noted that vitamin $E$ only delayed oxidation, but that the maximum amount of dienes formed was not affected, in agreement with results from Wander et $a{ }^{11}{ }^{11}$. On the other hand, this delay may also be important in vivo, since 
Regnstrōm et al. ${ }^{14}$ showed an inverse relation between the lagtime for coppercatalyzed LDL oxidation in vitro and quantitative estimates of global coronary atherosclerosis in humans. As compared with fish oil supplementation alone, extra vitamin $E$ supplementation did not significantly affect the rate of oxidation, but recent dose-response studies show that dietary dosages of $400 \mathrm{IU} /$ day $^{15}$ or $800 \mathrm{IU} /$ day $^{16}$ may be necessary to detect significant effects on the rate of oxidation. Nonetheless, it is still unclear how vitamin $E$ in high dietary dosages ${ }^{15,16}$ may affect the rate of oxidation, since all vitamin $E$ is already consumed during the lag phase ${ }^{13,17,18}$.

With respect to LDL oxidation in vitro, our studies (Chapter 2 and 3 ) do not provide clear evidence that the intake of vitamin $E$ should be increased during fish oil supplementation. On the other hand, a fish oil-induced decrease in lagtime (Chapter 2, and refs 7 and 11), which was however not noticed in the study in Chapter 3, may be counteracted by vitamin $E$ supplementation (Chapter 2, and ref. 11). Croft et al. ${ }^{19}$ even reported a prooxidative effect of vitamin $E$, since the cholesterol-standardized vitamin $E$ content of LDL correlated positively with the rate of oxidation and with the amount of dienes formed during LDL oxidation in vitro. Similar correlations were found for the protein-standardized vitamin $E$ content ${ }^{12}$. However, in not supplemented subjects, these correlations may be related to the PUFA content of LDL, since an increase in PUFA is correlated with an increase in vitamin $E^{20}$, and an increase in PUFA may also be correlated with increases in rate of oxidation and the amount of dienes (see above).

\section{Endurance exercise}

Strenuous endurance exercise did not affect the lagtime before onset of rapid oxidation of LDL in vitro or the rate of oxidation, but decreased the amount of conjugated dienes formed (Chapter 3 ). This may indicate that the relative amounts of PUFA in the native LDL had decreased ${ }^{2,12}$. Sumikawa et al. ${ }^{21}$ showed that exercise decreased the proportion PUFA in red blood cell phospholipids of untrained subjects. Although such a decrease may be a result of lipid peroxidation in vivo, perhaps due to decreased antioxidant protection (see below), alternative explanations cannot be excluded. Therefore, additional studies are required for confirmation. Increasing the supply of substrate for lipid peroxidation by fish oil supplementation, did not alter exercise-induced changes in LDL oxidation. 
Percutaneous trans/uminal coronary angioplasty

LDL oxidation in vitro was also studied in patients undergoing percutaneous transluminal coronary angioplasty (PTCA), a medical procedure in which blood flow in an obstructed coronary artery is restored by ballooning (Chapter 6). The reoxygenation, which may be associated with free radical production, did not affect the lagtime and the maximum amount of dienes during oxidation of LDL in vitro. The maximum rate of oxidation decreased during the ten minutes after the first balloon inflation, an effect observed in both venous and coronary arterial samples. In addition, no change in plasma lipid hydroperoxide levels were noticed, ten minutes after the first ballooning. These results do not indicate that PTCA in humans increases LDL oxidation in vitro.

Extrapolation of copper-catalyzed oxidation of LDL in vitro to in vivo processes

Research in animals and humans has provided several supportive lines of evidence for the occurrence of oxidation of LDL in vivo, and for its role in atherosclerosis as postulated by Steinberg et al. (see ref. 22, and refs therein). However, due to ethical and logistical problems, development of in vitro techniques was necessary in human studies. In our studies, the susceptibility of LDL to oxidation was studied in vitro with copper as prooxidant, and under conditions where the LDL are not protected by all interacting (water-soluble) antioxidant systems normally present in the human body. Nevertheless, in vitro copper-oxidized LDL greatly resembles LDL extracted from human and rabbit atherosclerotic lesions ${ }^{23,24}$, IgG isolated from these lesions recognizes copper-oxidized LDL but not native $\operatorname{LDL}^{25}$, and the degree of atherosclerosis in humans is correlated with copper-catalyzed oxidation of $\mathrm{LDL}^{14}$. Although atherosclerosis is a multifactorial process, $L D L$ oxidation in vitro may therefore prove a useful technique for future research in this field. 


\section{Plasma lipid-soluble antioxidant levels}

\section{Fish oil supplementation and endurance exercise}

Fish oil supplementation (Chapter 2 and 3 ) did not affect plasma lipid-soluble antioxidant levels. Although only LDL- $\alpha$-tocopherol levels were reported in the first study (Chapter 2), the whole spectrum of lipid-soluble antioxidants were later analyzed (not reported), in an attempt to find an explanation for the discrepancies between the two fish oil supplementation studies. However, plasma lipid-soluble antioxidant levels and responses were comparable. The increase in the amount of highly unsaturated fatty acids apparently does not reduce lipid-soluble antioxidant levels, that is, at least in plasma. Intense endurance exercise for 1 hour without intake of nutrients, on the other hand, decreased plasma tocopherol and carotenoid levels adjusted for hemoconcentration (Chapter 3 ). Although the decreases in antioxidants only provide indirect supportive evidence for oxidative stress during exercise, we also noticed a decrease in red blood cell deformability. Such a decrease has been associated with oxidative stress as well ${ }^{26}$.

\section{Normal pregnancy}

During pregnancy, plasma levels of various lipids increase, and we therefore studied whether the increase in PUFA contained in these lipids compromised antioxidant defences (Chapter 4). However, plasma tocopherol and lutein levels increased by more than $40 \%$ from the 1 st to the 3rd trimester, which was even more than the increase in plasma unsaturation as estimated by the absolute phospholipid PUFA unsaturation index $\left(\mathrm{Ul}_{\mathrm{abs}}\right)$. Although plasma levels of most carotenoids remained constant during pregnancy, there was a significant decline in B-carotene levels. Furthermore, since the $\mathrm{UI}_{\mathrm{abs}}$ increased during pregnancy, carotenoid/UI $\mathrm{abs}_{\mathrm{ab}}$ ratios decreased. The different responses of the antioxidants during pregnancy indicate that measurements of total tocopherol (vitamin E) or total carotenoid levels may not provide an accurate reflection of lipid-soluble antioxidant responses. Besides for B-carotene, little information is available on the antioxidant capacity of other carotenoids (for reviews, see refs 27, 28 and 29). Although carotenoid are present in much lower concentrations than, for example $\alpha$-tocopherol, Di Mascio et $a l^{30}$ showed that the higher singlet oxygen quenching capacity of carotenoids compensates for this difference. However, the role of singlet oxygen in lipid 
peroxidation is still unclear ${ }^{31}$, and effects observed in in vitro models might not be relevant in vivo.

Remarkably, whereas most plasma antioxidant levels did not change from the 3rd trimester to postpartum, there was a distinct decrease in $\delta$-tocopherol and $B+\gamma$-tocopherol levels (Chapter 4 ). The decrease in $B+\gamma$-tocopherol levels was also noticed in another group of pregnant women (Chapter 5 ). Future studies may indicate whether this decline occurred during the last phase of pregnancy, or whether, for example, oxidative stress during labor diminished plasma levels of the less abundant tocopherol isomers.

In neonates just after birth, plasma antioxidant levels were considerably lower than levels found in adults (Chapter 4 and 5). Although plasma lipid levels were also lower in neonates our data indicated that antioxidant levels adjusted for phospholipid or phospholipid unsaturation are still lower in neonates. However, the plasma levels of the most abundant lipid-soluble antioxidant, $\alpha$-tocopherol, in neonates did not correlate with levels in the mothers, and newboms from mothers with low plasma $\alpha$-tocopherol levels, had similar levels as well-nourished mothers (see discussion Chapter 4). This indicates that supplementation of mothers with $\alpha$-tocopherol will probably not improve the neonatal antioxidant status. For several carotenoids, but certainly not for phytofluene, neonatal levels appeared to be influenced by maternal levels, and this observation may have implications for future studies in pregnant women and their newborns.

\section{Pregnancy complicated with pregnancy-induced hypertension}

In pregnant women suffering from mild PIH, plasma lipid-soluble antioxidant levels during the 3rd trimester of pregnancy were similar to 3rd trimester plasma levels in women with uncomplicated pregnancies (Chapter 5). Others already found similar 3rd trimester vitamin $E$ (mainly $\alpha$-tocopherol) levels in uncomplicated pregnancies and pregnancies complicated with mild $\mathrm{PIH}^{32,33}$, but we showed that plasma levels of the less abundant tocopherols and various carotenoids were also similar. Although plasma antioxidant levels in women with PIH decreased from the 3rd trimester to postpartum, plasma unsaturation, as estimated by the phospholipid PUFA unsaturation index, decreased as well. Furthermore, neonatal plasma lipid-soluble antioxidant levels were unaffected by maternal $\mathrm{PIH}$. We, therefore, did not find a relation between the plasma lipid-soluble antioxidant status and mild PIH. Studies in women with severe 
$\mathrm{PIH}$, have presented inconsistent results with respect to plasma vitamin $E$ levels ${ }^{32,33}$, but the tendency to decreased antioxidant levels from the 3rd trimester to postpartum in women with mild PIH in our study (Chapter 5) merits further investigation of lipidsoluble antioxidant levels in severe PIH. On the other hand, a recent study showed that dietary supplementation with $300 \mathrm{mg} /$ day of vitamin $\mathrm{E}$ in women with PIH did not affect fetal mortality ${ }^{34}$. However, since vitamin $E$ was given during the 3 rd trimester after diagnosis of $\mathrm{PIH}$, this may have been too late to influence fetal outcome.

\section{Percutaneous transluminal coronary angioplasty}

During the first ten minutes of PTCA, LDL tocopherol levels increased in coronary arterial plasma, but not in venous samples (Chapter 6 ). In other procedures associated with ischemia and reperfusion, decreased $\alpha$-tocopherol levels in heart tissue ${ }^{35,36}$ and pulmonary artery plasma ${ }^{37}$ have been noticed. However, the periods of ischemia in these studies were much longer than those used during PTCA in the present study, and results may therefore not be comparable. Carotenoid levels also appeared to increase in arterial samples, but decreased in venous samples. However, this decrease was only small, and the increased coronary arterial antioxidant levels, the decrease in rate of in vitro oxidation of LDL, and absence of increased plasma levels of lipid hydroperoxides do not support that PTCA in humans is associated with lipid peroxidation shortly after the start of the procedure. Although LDL levels of the less abundant tocopherol isomers were decreased two days after PTCA, this did not affect LDL oxidation in vitro or plasma lipid hydroperoxide levels.

\section{Conclusions}

In situations potentially subjected to oxidative stress (i.e. an imbalance between prooxidants and antioxidants), like supplementation with highly unsaturated n-3 fatty acids, exercise, pregnancy, mild PIH and PTCA, we found little or inconsistent evidence for increased systemic lipid peroxidation. Although this may indicate that the human body is sufficiently capable of counteracting (mild) oxidative stress, local effects may not have been detectable in plasma. However, in most situations it is impossible to study local events in humans. In the studies described, we have investigated lipid peroxidation by using different techniques simultaneously, several of which have 
established meaning in lipid peroxidation research.

The method of copper-catalyzed oxidation of LDL in vitro has provided the opportunity to view the effects of moderate interventions on LDL oxidation characteristics, which may be relevant for the in vivo situation. By using the rapid short-run isolation (Chapter 3) and gel filtration (Chapter 3 and 6) techniques, the disadvantage of time-consuming preparation of LDL has been overcome, and a reasonable number of samples can be analyzed within a short period. Furthermore, the possible introduction of analytical artefacts, as during extensive preparation procedures, is reduced. However, lipid peroxidation should not only be based on LDL oxidation in vitro, but should simultaneously be estimated with other techniques as well. The method of analysis of antioxidants in one single run, as developed by Hess et al..$^{38}$, provides plasma concentrations of 10 lipid-soluble antioxidants within a short period. It should be noted, however, that the antioxidant capacity of these molecules is often based on in vitro activity, and, especially with regard to the carotenoids and retinol, needs more proof for their action in vivo. Although lipid-soluble antioxidants may be very important in lipid environments of, for example, membranes, other antioxidant systems may also contribute to antioxidant defences.

\section{Future research}

Several 'indicators' have been developed to estimate the extent of lipid peroxidation in the human body. The very simple assay based on the reaction with thiobarbituric acid may be suitable for rough estimates of lipid peroxidation in patients, but lacks sensitivity and specificity to detect small changes in healthy subjects (see also Chapter 1). Recently introduced commercially available assays to easily and more specifically measure lipid hydroperoxides concentrations in plasma or serum, however, have detection limits of $2 \mu \mathrm{moVl}$ (LPO-CC, Kamiya Biomedical Company, Thousand Oaks, CA, USA) and $2.5 \mu \mathrm{mol} /$ (LPO-586, Bioxytech, Bonneuil sur Mame, France), and are therefore not suitable for plasmas of healthy subjects. $A$ iodometric method, developed for detection of lipid hydroperoxides in $\mathrm{LDL}^{39}$, was recently used for plasma $^{40}$. We (results not shown) and others ${ }^{41,42}$, however, could not detect lipid hydroperoxides in plasma of healthy subjects using iodometry. A more sensitive, method using HPLC and chemiluminescence detection, could detect free fatty acid and 
cholesterol ester hydroperoxides at picomole levels, but could not be used for detection of phospholipid hydroperoxides, and is very laborious ${ }^{43}$. However, to study lipid hydroperoxide concentrations in plasma of healthy subjects it seems inevitable to develop and use very sensitive methods.

To include the influence of water-soluble antioxidants and antioxidant proteins, assays that measure the total antioxidant capacity of serum or plasma in response to a prooxidant in vitro have been developed. However, result obtained with these assays should also be viewed with care. They may be affected by the medium in which the assay is performed, and may depend on the kind of prooxidant the plasma or serum has to inhibit. Furthermore, the antioxidant capacity assay may be affected by pure antioxidant standards, but this does not mean that changes in plasma antioxidant levels will be detected by the assay. For example, Rice-Evans and Miller ${ }^{44}$ showed that antioxidants found in plasma, like $\alpha$-tocopherol and vitamin $C$, when added as pure substances, affected their recently developed assay for the total antioxidant status in plasma and body fluids. However, they also showed that dietary supplementation with $300 \mathrm{mg}$ of $\alpha$-tocopherol, $250 \mathrm{mg}$ of vitamin C, or $15 \mathrm{mg}$ B-carotene per day for 8 weeks, increased plasma levels by $70 \%, 30 \%$, and $250 \%$, respectively, but did not affect the 'total antioxidant status' ${ }^{\mathbf{4 5}}$.

Recent studies have focussed again on LDL oxidation in vivo. Virella et al. ${ }^{46}$ detected antibodies against copper-oxidized LDL in human sera. They found that antibody levels were similar in healthy subjects and subjects with coronary heart disease. Holvoet et al. ${ }^{47}$ also measured similar plasma lgG autoantibody titers for MDA-modified LDL in healthy subjects and subjects with chronic stable angina pectoris, carotid atherosclerosis, or myocardial infarction. Using a murine monoclonal antibody, they also detected MDA-modified LDL in carotid atherosclerotic lesions, confirming previous research ${ }^{23,48}$, but, in addition, demonstrated MDA-modified LDL in plasma. With this new technique, they found similar plasma levels of MDA-modified LDL in patients with chronic stable angina pectoris as compared with controls, but higher levels in patients with carotid atherosclerosis, and in patients early after the onset of myocardial infarction. Although these sophisticated techniques provide important evidence for modification of LDL in vivo, they have only been used in patients. It would be challenging to use these techniques to study effects of dietary manipulations. 


\section{REFERENCES}

1. Parthasarathy S, Khoo JC, Miller E, Barnett J, Witztum JL, Steinberg D. Low density lipoprotein rich in oleic acid is protected against oxidative modification: Implications for dietary prevention of atherosclerosis. Proc Natl Acad Sci USA 1990; 87:3894-3898.

2. Reaven P, Parthasarathy S, Grasse BJ, Miller E, Almazan F, Mattson FH, Khoo JC, Steinberg D, Witztum JL. Feasibility of using an oleate-rich diet to reduce the susceptibility of low-density lipoprotein to oxidative modification in humans. Am J Clin Nutr 1991; 54:701-706.

3. Reaven P, Parthasarathy S, Grasse BJ, Miller E, Steinberg D, Witztum JL. Effects of oleate-rich and linoleate rich diets on the susceptibility of low density lipoprotein to oxidative modification in mildly hypercholesterolemic subjects. J Clin invest 1993; 91:668-676.

4. Abbey M, Belling GB, Noakes M, Hirata F, Nestel PJ. Oxidation of low-density lipoproteins: intraindividual variability and the effect of dietary linoleate supplementation. Am J Clin Nutr 1993; 57:391-398.

5. Harats D, Dabach Y, Hollander G, Ben-Naim M, Schwartz R, Berry EM, Stein O, Stein Y. Fish oil ingestion in smokers and nonsmokers enhances peroxidation of plasma lipoproteins. Atherosclerosis 1991; 90:127-139.

6. Lussier-Cacan S, Dubreuil-Quidoz S, Roederer G, Leboeut N, Boulet L, de Langavant GC, Davignon J, Naruszewicz M. Influence of probucol on enhanced LDL oxidation after fish oil treatment of hypertriglyceridemic patients. Arterioscler Thromb 1993; 13:1790-1797.

7. Suzukawa M, Abbey M, Howe PR, Nestel PJ. Effects of fish oil fatty acids on low density lipoprotein size, oxidizability, and uptake by macrophages. J Lipid Res 1995; 36:473-484.

8. Popp-Snijders C, Schouten JA, de Jong AP, van der Veen EA. Effect of cod-liver oil on the lipid composition of human erythrocyte membranes. Scand J Clin Lab Invest 1984; 44:39-46.

9. Sanders TAB, Hinds $A$. The influence of a fish oil high in docosahexaenoic acid on plasma lipoprotein and vitamin $\mathrm{E}$ concentrations and haemostatic function in healthy male volunteers. $\mathrm{Br}$ J Nutr 1992; 68:163-173.

10. Nenseter MS, Rustan AC, Lund-Katz S, Søyland E, Mælandsmo G, Phillips MC, Drevon CA. Effect of dietary supplementation with n-3 polyunsaturated fatty acids on physical properties and metabolism of low density lipoprotein in humans. Arterioscler Thromb 1992; 12:369-379.

11. Wander RC, Du S-H, Ketchum SO, Rowe KE. Effects of interaction of RRR- $\alpha$-tocopheryl acetate and fish oil on low-density-lipoprotein oxidation in postmenopausal women with and without hormone-replacement therapy. Am J Clin Nutr 1996; 63:184-193.

12. Kleinveld HA, Naber AHJ, Stalenhoef AFH, Demacker PNM. Oxidation resistance, oxidation rate, and extent of oxidation of human low-density lipoprotein depend on the ratio of oleic acid content to linoleic acid content: studies in vitamin E deficient subjects. Free Rad Biol Med 1993; 15:273-280.

13. Esterbauer $H$, Striegl $G$, Puhl $H$, Rotheneder $M$. Continuous monitoring of in vitro oxidation of human low density lipoprotein. Free Rad Res Commun 1989; 6:67-75.

14. Regnstrōm J, Nilsson J, Tomvall P, Landou C, Hamsten A. Susceptibility to low-density lipoprotein oxidation and coronary atherosclerosis in man. Lancet 1992; 339:1183-1186.

15. Princen HMG, van Duyvenvoorde W, Buytenhek R, van der Laarse A, van Poppel G, Gevers Leuven JA, van Hinsbergh WWM. Supplementation with low doses of vitamin E protects LDL from lipid peroxidation in men and women. Arterioscler Thromb Vasc Biol 1995; 15:325-333.

16. Jialal I, Fuller CJ, Huet BA. The effect of $\alpha$-tocopherol supplementation on LDL oxidation. A dose-response study. Arterioscler Thromb Vasc Biol 1995; 15:190-198. 
17. Steinbrecher UP, Zhang $\mathrm{H}$, Lougheed $M$. Role of oxidative modified LDL in atherosclerosis. Free Rad Biol Med 1990; 9:155-168.

18. Jessup W, Rankin SM, De Whalley CV, Hoult JRS, Scott J, Leake DS. $\alpha$-Tocopherol consumption during low-density-lipoprotein oxidation. Biochem J 1990; 265:399-405.

19. Croft KD, Williams $P$, Dimmitt $S$, Abu-Amsha R, Beilin LJ. Oxidation of low-density lipoproteins: effect of antioxidant content, fatty acid composition and intrinsic phospholipase activity on susceptibility to metal ion-induced oxidation. Biochim Biophys Acta 1995; 1254:250-256.

20. Esterbauer $H$, Dieber-Rotheneder $M$, Striegl $G$, Waeg $G$. Role of vitamin $E$ in preventing the oxidation of low-density lipoprotein. Am J Clin Nutr 1991; 53:314S-321S.

21. Sumikawa $K$, Mu Z, Inoue $T$, Okochi $T$, Yoshida $T$, Adachi $K$. Changes in enthrocyte membrane phospholipid composition induced by physical training and physical exercise. Eur J Appl Physiol 1993; 67:132-137.

22. Steinberg D, Parthasarathy S, Carew TE, Khoo JC, Witztum JL. Beyond cholesterol. Modifications of low-density lipoprotein that increase its atherogenicity. N Engl J Med 1989; 320:915-924.

23. Ylā-Herttuala S, Palinski W, Rosenfeld ME, Parthasarathy S, Carew TE, Butler S, Witztum JL, Steinberg D. Evidence for the presence of oxidatively modified low density lipoprotein in atherosclerotic lesions of rabbit and man. J Clin Invest 1989; 84:1086-1095.

24. Ylä-Herttuala S, Palinski W, Rosenfeld ME, Steinberg D, Witztum JL. Lipoproteins in normal and atherosclerotic aorta. Eur Heart J 1990; 11(suppl E):88-99.

25. Ylä-Herttuala S, Palinski W, Butler SW, Picard S, Steinberg D, Witztum JL. Rabbit and human atherosclerotic lesions contain IgG that recognizes epitopes of oxidized LDL. Arterioscler Thromb 1994; 14:32-40.

26. Pfafferott C, Meiselman HJ, Hochstein P. The effect of malonyldialdehyde on erythrocyte deformability. Blood 1982; 59:12-15.

27. Sies $\mathrm{H}$, Stahl $\mathrm{W}$, Sundquist $A R$. Antioxidant function of vitamins. Vitamins $E$ and $C$, beta-carotene, and other carotenoids. Ann N Y Acad Sci 1992; 669:7-20.

28. Palozza P, Krinsky NI. Antioxidant effects of carotenoids in vivo and in vitro: An overview. Meth Enzymol 1992; 213:403-420.

29. Buettner GR. The pecking order of free radicals and antioxidants: lipid peroxidation, $\alpha$-tocopherol, and ascorbate. Arch Biochem Biophys 1993; 300:535-543.

30. Di Mascio P, Kaiser S, Sies H. Lycopene as the most efficient biological carotenoid singlet oxygen quencher. Arch Biochem Biophys 1989; 274:532-538.

31. Halliwell B, Gutteridge JMC. Role of free radicais and catalytic metal ions in human disease: An overview. Meth Enzymol 1990; 186:1-85.

32. Wang YP, Walsh SW, Guo JD, Zhang JY. The imbalance between thromboxane and prostacyclin in preeclampsia is associated with an imbalance between lipid peroxides and vitamin $E$ in matemal blood. Am J Obstet Gynecol 1991; 165:1695-1700.

33. Uotila JT, Tuimala RJ, Aamio TM, Pyykkō KA, Ahotupa MO. Findings on lipid peroxidation and antioxidant function in hypertensive complications of pregnancy. Br J Obstet Gynaecol 1993; 100:270-276.

34. Stratta $P$, Canavese C, Porcu M, Dogliani M, Todros T, Garbo E, Belliardo F, Maina A, Marozio L, Zonca M. Vitamin E supplementation in preeclampsia. Gynecol Obstet Invest 1994; 37:246-249.

35. Barsacchi R, Pelosi G, Maffei S, Baroni M, Salvatore L, Ursini F, Verunelli F, Biagini A. Myocardial vitamin $E$ is consumed during cardiopulmonary bypass: indirect evidence of free radical generation in human ischemic heart. Int J Cardiol 1992; 37:339-343.

36. Weisel RD, Mickle DAG, Finkle CD, Tumiati LC, Madonik MM, Ivanov J, Burton GW, Ingold KU. Myocardial free-radical injury after cardioplegia. Circulation 1989; 80(suppl III):III-14-III-18. 
37. Murphy ME, Kolvenbach $R$, Aleksis $M$, Hansen $R$, Sies $H$. Antioxidant depletion in aortic crossclamping ischemia: Increase of the plasma $\alpha$-tocopheryl quinone/ $\alpha$-tocopherol ratio. Free Rad Biol Med 1992; 13:95-100.

38. Hess D, Keller HE, Oberlin B, Bonfanti $R$, Schũep W. Simultaneous determination of retinol, tocopherols, carotenes and lycopene in plasma by means of high-pertormance liquid chromatography on reversed phase. Int J Vitam Nutr Res 1991; 61:232-238.

39. E\}-Saadani M, Esterbauer H, El-Sayed M, Goher M, Nassar AY, Jūrgens G. A spectrophotometric assay for lipid peroxides in serum lipoproteins using a commercially available reagent. $J$ Lipid Res 1989; 30:627-630.

40. Görōg PG, Kotak DC, Kovacs IB. Simple and specific test for measuring lipid peroxides in plasma. $J$ Clin Pathol 1991; 44:765-767.

41. Wieland E, Schettler V, Diedrich F, Schutf-Wemer P, Oellerich $M$. Determination of lipid hydroperoxides in serum iodometry and high performance liquid chromatography compared. Eur $\mathrm{J}$ Clin Chem Clin Biochem 1992; 30:363-369.

42. Darrow RA, Organisciak DT. An improved spectrophotometric triiodide assay for lipid hydroperoxides. Lipids 1994; 29:591-594.

43. Yamamoto $\mathrm{Y}$, Brodsky $\mathrm{MH}$, Baker JC, Ames BN. Detection and characterization of lipid hydroperoxides at picomole levels by high-performance liquid chromatography. Anal Biochem 1987; 160:7-13.

44. Rice-Evans C, Miller NJ. Total antioxidant status in plasma and body fluids. Meth Enzymol 1994; 234:279-293.

45. Calzada C, Bizzotto M, Paganga G, Miller NJ, Bruckdorfer KR, Diplock AT, Rice-Evans CA. Levels of antioxidant nutrients in plasma and low density lipoproteins: a human volunteer supplementation study. Free Rad Res 1995; 23:489-503.

46. Virella G, Virella I, Leman RB, Pryor MB, Lopes-Virella MF. Anti-oxidized low-density lipoprotein antibodies in patients with coronary heart disease and normal healthy volunteers. Int $\mathrm{J}$ Clin Lab Res 1993; 23:95-101.

47. Holvoet P, Perez G, Zhao Z, Brouwers E, Bernar H, Collen D. Malondialdehyde-modified low density lipoproteins in patients with atherosclerotic disease. J Clin Invest 1995; 95:2611-2619.

48. Palinski W, Rosenfeld ME, Ylä-Herttuala S, Gurtner GC, Socher SS, Butler SW, Parthasarathy S, Carew TE, Steinberg D, Witztum JL. Low density lipoprotein undergoes oxidative modification in vivo. Proc Natl Acad Sci USA 1989; 86:1372-1376. 


\section{SAMENVATTING}

Vrije zuurstof radicalen spelen een belangrijke rol in vele biologische processen in de mens. Het zijn echter zeer reactieve deeltjes, waardoor schade aan weefsels kan ontstaan. Antioxydantenzymen en andere antioxydantverbindingen in het lichaam kunnen deze reactieve deeltjes weer onschadelijk maken.

Meervoudig onverzadigde vetzuren (MOV) zijn zeer gevoelig voor aanvallen van vrije zuurstof radicalen door de aanwezigheid van twee of meer dubbele bindingen (Hoofdstuk 1). Deze gevoeligheid neemt toe bij een toename van het aantal dubbele bindingen. Dit proces, en de daarop volgende peroxydatie van het vetzuur onder invloed van zuurstof wordt 'lipidenperoxydatie' genoemd. Gedurende lipidenperoxydatie worden lipoperoxyden gevormd die op hun beurt andere vetzuren kunnen aanvallen, en zo een ketting-reactie veroorzaken. Dit kan leiden tot beschadiging van celmembranen, waarin deze MOV zich bevinden. De relatieve hoeveelheid en de soort MOV in membranen wordt onder andere bepaald door de vetzuursamenstelling van de voeding. Antioxydanten, zoals vitamine $E$ uit de voeding, kunnen de ketting-reactie van lipidenperoxydatie stoppen. Lipidenperoxydatie is dus afhankelijk van de vorming van vrije zuurstof radicalen, van de beschikbaarheid van MOV, van de hoeveelheid dubbele bindingen in de MOV, en van de aanwezigheid van antioxydanten.

Lipidenperoxydatie vindt niet alleen plaats in cellulaire membranen, maar ook in bijvoorbeeld lipoproteïnen. Resultaten van veel recente onderzoeken geven aan dat het ontstaan en/of de progressie van atherosclerose mogelijk een oorzaak vinden in lipidenperoxydatie van lage dichtheid lipoproteīnen (LDL) (Hoofdstuk 1).

Aangezien lipidenperoxydatie afhankelijk is van de hoeveelheid beschikbare dubbele bindingen in MOV, werd de invloed van een verhoging van de hoeveelheid sterk onverzadigde $M O V$ in de voeding op de oxydatie-gevoeligheid van LDL onderzocht (Hoofdstuk 2). Gezonde proefpersonen kregen gedurende 3 weken een supplement bij hun voeding, bestaande uit visolie, een olie met veel vetzuren met 5 en 6 dubbele bindingen. Indien men daama LDL isoleerde uit het bloed van die proefpersonen, en het vervolgens oxydeerde met koper-ionen, dan werd er meer oxydatie-produkt (geconjugeerde diēnen) gevormd. In een groep die naast de visolie tevens vitamine $\mathrm{E}$ ontving, duurde het langer voordat de oxydatie in vitro op gang kwam, maar de hoeveelheid diënen was uiteindelijk even sterk verhoogd als bij de 
proefpersonen die alleen visolie kregen. Uit deze proef werd geconcludeerd dat de sterk onverzadigde vetzuren uit visolie de in vitro oxydeerbaarheid van LDL verhogen, maar dat vitamine $E$ in de voeding de weerstand tegen dit proces verbetert.

Er zijn aanwijzingen dat visolie een positieve invioed heeft op het fysieke prestatievermogen. Inbouw van de vetzuren uit visolie in membranen van rode bloedcellen zou de flexibiliteit van deze cellen kunnen bevorderen, en daarmee de voorziening van zuurstof aan de spieren. Echter fysieke inspanning verhoogd de vorming van zuurstof radicalen, en een extra verhoging van de mate van lipidenperoxydatie door visolie, zoals waargenomen in Hoofdstuk 2, zou niet wenselijk zijn.

Om het effect van inspanning per se te bepalen werd eerst het fysieke prestatievermogen, de flexibiliteit van rode bloedcellen en de mate van lipidenperoxydatie gemeten in 24 getrainde atleten voor dat deze een supplement kregen (Hoofdstuk 3). Een tijdrit van 1 uur op een fiets-ergometer resulteerde in een daling van de flexibiliteit van de rode bloedcellen. Gedurende de tijdrit daalde ook de hoeveelheid antioxydanten (tocoferolen en carotenoìden) in plasma, maar onduidelijk is of dit gerelateerd is aan lipidenperoxydatie, aan een verschuiving van antioxydanten van het plasma naar weefsels, of aan een combinatie hiervan. Het meten van meer parameters van lipidenperoxydatie zal daarom noodzakelijk zijn.

Gedurende 3 weken ontvingen vervolgens twee groepen van 8 atleten een dagelijks supplement bestaande uit visolie, en van die twee groepen kreeg één groep van 8 tevens vitamine E. De derde groep van 8 ontving een placebo supplement. Suppletie met visolie veroorzaakte echter geen verandering van de flexibiliteit van de rode bloedcellen. In tegenstelling tot de bevindingen in Hoofdstuk 2 veroorzaakte suppletie met visolie bij de atleten (Hoofdstuk 3) geen stijging van de vorming van geconjugeerde diēnen tijdens oxydatie van LDL in vitro. Voor de tegenstrijdige resultaten van Hoofdstuk 2 en 3, met betrekking tot LDL oxydatie in vitro, is tot op heden echter nog geen verklaring gevonden. Na suppletie met visolie werd er geen verbetering van de prestatie waargenomen, maar waren er ook geen aanwijzingen dat de combinatie van inspanning en visolie-suppletie de mate van lipidenperoxydatie extra verhoogt.

Gedurende de zwangerschap stijgen de plasma concentraties van lipiden. Aangezien de hoeveelheid MOV in plasma dan ook stijgt, werd onderzocht of de 
hoeveelheid antioxydanten in plasma zich aanpast aan de verhoogde concentratie MOV (Hoofdstuk 4). Verder werd ook gekeken naar de plasma antioxydant concentraties van de pasgeborenen, omdat andere onderzoeken hebben aangetoond dat de plasma vitamin E concentratie veel lager is dan die van de moeder. Alhoewel ook de plasma concentratie van veel lipiden lager is dan bij de moeder, is de relatieve bijdrage van sterk onverzadigde en oxydatie-gevoelige MOV in fosfolipiden groter.

Van het eerste naar het derde trimester van de zwangerschap stegen de plasma concentraties van tocoferolen en de carotenoïd luteïne met meer dan 40\%. Dit was meer dan de stijging van de mate van onverzadiging van het plasma, berekend aan de hand van de stijging van MOV in fosfolipiden en het aantal dubbele bindingen in die MOV. De B-caroteen concentratie in plasma daalde echter met $19 \%$, terwijl de concentraties van de andere carotenoïden constant bleven gedurende de zwangerschap. Hierdoor daalde, van het eerste naar het derde trimester, de ratio van deze carotenoïden ten opzichte van de mate van onverzadiging.

$\mathrm{Na}$ de bevalling waren de plasma concentraties van $\alpha$-tocoferol en de carotenoïden vergelijkbaar met die in het derde trimester. De plasma concentraties van $\delta$ - en $B+\gamma$-tocoferol waren echter significant lager na de bevalling ten opzichte van waarden in derde trimester. Het is onduidelijk of dit effect optreedt gedurende de laatste fase van de zwangerschap, of dat de (oxydatieve) stress gedurende de bevalling hierop van invloed is.

In pasgeborenen waren de plasma antioxydant concentraties veel lager dan die van de moeder. Plasma concentraties van tocoferolen en carotenoïden waren ook significant lager indien ze gecorrigeerd werden voor de lagere mate van onverzadiging van het neonatale plasma. Er werd nagenoeg geen fytoflueen aangetroffen in plasma van pasgeborenen. Deze carotenoïd wordt dus tijdens de zwangerschap niet van de moeder op het kind overgebracht. Verder bleek dat $\beta+\gamma$-tocoferol en veel carotenoïd concentraties in neonataal plasma correleerden met die in moederlijk plasma, maar dat dit niet gold voor $\delta$-tocoferol, $\alpha$-tocoferol, lycopeen en retinol.

Deze studie heeft getoond dat de stijging van de plasma concentraties oxydatiegevoelige MOV gedurende de zwangerschap, gepaard gaat met een stijging van de concentraties van tocoferol en luteïne. In hoeverre de stijging van deze antioxydanten een potentiële stijging van lipidenperoxydatie kunnen voorkomen, zal additioneel onderzoek moeten uitwijzen. Alhoewel de rol van carotenoïden als antioxydanten in vivo ter discussie staat, kan de daling van de plasma B-caroteen concentratie 
tijdens de zwangerschap van biologische belang zijn. De daling van bepaalde tocoferol isomeren na de bevalling, en de lage neonatale plasma concentraties van tocoferolen en carotenoïden geven aan dat onderzoek naar parameters van lipidenperoxydatie rond de tijd van bevalling noodzakelijk is.

Resultaten van recente onderzoeken duiden erop dat kenmerken van zwangerschapshypertensie mede verklaard zouden kunnen worden door een verstoorde balans tussen lipidenperoxydatie en antioxydant status. Vooral de rol van vetoplosbare antioxydanten, zoals vitamine $\mathrm{E}$, werd hierbij benadrukt. Echter niet in alle onderzoeken werden verlaagde plasma vitamine $E$ concentraties gevonden bij zwangerschapshypertensie. Verder werd er geen rekening gehouden met veranderingen in de vetzuur-status. Daarom werden de concentraties van verschillende vetoplosbare antioxydanten gemeten in plasma van 23 vrouwen met zwangerschapshypertensie gedurende het derde trimester en postpartum, en vergeleken met 23 zwangere vrouwen zonder zwangerschapshypertensie (Hoofdstuk 5). Tevens werd onderzocht of de zwangerschapshypertensie bij de moeder van invloed was op de antioxydant status van de pasgeborene. Plasma antioxydant concentraties werden gerelateerd aan de mate van onverzadiging van fosfolipiden om te corrigeren voor veranderingen in MOV-status, en omdat de hoge mate van onverzadiging van fosfolipiden, membranen mogelijk gevoelig maakt voor lipidenperoxydatie. Er zijn aanwijzingen dat beschadiging van endotheliale cellen een rol speelt in de pathogenese van zwangerschapshypertensie.

In het derde trimester waren de plasma concentraties van de gemeten tocoferolen, carotenoïden en retinol vergelijkbaar in vrouwen met of zonder zwangerschapshypertensie. $\mathrm{Na}$ de bevalling was de plasma concentratie van $\beta+\gamma$-tocoferol in de controle groep $5 \%$ lager dan die in het derde trimester, een effect dat ook werd waargenomen in de studie beschreven in Hoofdstuk 4. In de groep vrouwen met zwangerschapshypertensie waren de postpartum plasma concentraties van verscheidene antioxydanten 15 tot 26 percent lager dan de concentraties in het derde trimester, hetgeen niet werd waargenomen in de controle groep. Na correctie voor veranderingen in de mate van onverzadiging, die verschillend waren in de twee groepen, was er echter geen onderscheid meer tussen de twee groepen. Uit onze studie bleek ook dat de antioxydant status van de pasgeborenen niet beïnvloed werd door de milde zwangerschapshypertensie van de moeder. Deze resultaten 
onderschrijven daarom niet dat zwangerschapshypertensie gepaard gaat met een verslechterde antioxydant status van moeder of kind. De vrouwen met zwangerschapshypertensie in onze studie hadden een relatief milde vorm van deze aandoening. Het is daarom mogelijk dat bij een zware vorm van zwangerschapshypertensie de antioxydant status sterker afwijkt van een controle groep.

Gedurende veel operatieve ingrepen worden arteriën tijdelijk afgesloten. Resultaten uit proeven met dieren geven aan dat de hernieuwde toevoer van bloed (reperfusie), en dus van zuurstof, naar het voorheen ischemische deel van de arterie een overmaat aan vrije zuurstof radicalen veroorzaakt, en dus mogelijk gevolgen heeft voor de mate van lipidenperoxydatie. In dieren werd aangetoond dat het toedienen van antioxydanten het herstel van de hartfunctie na ischemie/reperfusie bespoedigt. Echter, metingen van verschillende indicatoren voor lipidenperoxydatie leverden tegenstrijdige resultaten op.

In mensen is oxydatieve stress na ischemie/reperfusie voomamelijk onderzocht gedurende operatieve ingrepen onder zeer variabele condities. Percutane transluminale coronaire angioplastiek (PTCA) is een routinematig uitgevoerde medische handeling onder goed-gestandaardiseerde omstandigheden, en zou dus een bruikbaar ischemie/reperfusie model voor oxydatieve stress in de mens kunnen zijn. Bij PTCA wordt een vernauwing in een kransslagader verwijd met behulp van een ballon-catheter. Deze procedure wordt gewoonlijk aangeduid met 'dotteren'.

In eerdere onderzoeken werd lipidenperoxydatie tijdens PTCA in mensen meestal onderzocht door het meten van produkten in plasma die reageren met thiobarbituurzuur (TBArs). Deze indirecte methode is echter kwalitatief en kwantitatief niet zeer betrouwbaar, hetgeen mogelijk de tegenstrijdige resultaten zou kunnen verklaren. Daarom werd in 13 patiēnten, voor en na PTCA, de plasma concentratie lipoperoxyden gemeten met een directe methode. Aangezien zuurstof radicalen kunnen reageren met MOV in LDL, zou modificatie van het LDL-deeltje een indicatie zijn voor het optreden van oxydatieve stress. Daarom werd de oxydatieve modificatie gemeten aan de hand van de lipoperoxyde en antioxydant concentratie van LDL, en de koper-geïnduceerde oxydatie-gevoeligheid van het deeltje (Hoofdstuk 6).

Tien minuten na de eerste verwijding (dilatatie) --soms zijn meer pogingen nodig om een klinisch acceptabel resultaat te krijgen--, waren de lipoperoxyde concentraties in plasma en LDL vergelijkbaar met concentraties voor het dotteren, zowel in veneuze 
monsters als in monsters uit de coronaire arterie. In het arteriële LDL stegen de antioxydant-concentraties (vooral $\alpha$-tocoferol), terwijl in veneus LDL de concentraties onveranderd bleven (tocoferolen) of licht daalden (carotenoïden). Tijdens de oxydatie van $L D L$ in vitro kwam de oxydatie even snel op gang in monster verzameld kort voor PTCA als in monsters verzameld 10 minuten na de eerste dilatatie. Alhoewel deze inloop periode, onder andere, bepaald wordt door de antioxydant concentratie in LDL, hadden de waargenomen veranderingen in de antioxydant concentratie dus geen statistisch significant effect op deze periode. $\mathrm{Na}$ de inloop periode treedt snelle oxydatie van vetzuren op. $\mathrm{Na}$ de eerste 10 minuten van de dotter-procedure verliep deze oxydatie langzamer dan ervoor. De hoeveelheid geconjugeerde diënen die gevormd werd wijzigde echter niet. Twee dagen na PTCA waren de meeste parameters vergelijkbaar met waarden enkele uren voor de PTCA, behalve de ongeveer $17 \%$ lagere $\delta$-tocoferol en $\beta+\gamma$-tocoferol concentraties in LDL. Desalniettemin zijn er weinig aanwijzingen gevonden dat er een verhoogde mate van lipidenperoxydatie optreedt tijdens PTCA.

In de algemene discussie (Hoofdstuk 7) worden de belangrijkste bevindingen van de studies in Hoofdstukken 2-6 kort uiteengezet. Geconcludeerd kan worden dat, ondanks het feit dat meerdere technieken tegelijkertijd werden gehanteerd die tevens reeds eerder succesvol zijn gebruikt voor het meten van lipidenperoxydatie, in de beschreven situaties van potentieel toegenomen oxydatieve stress slechts weinig aanwijzingen voor toegenomen lipidenperoxydatie zijn waargenomen. Interessant zou zijn of nieuwe 'in vivo'-technieken, zoals het meten van plasma antistoffen tegen geoxydeerd LDL dit zouden bevestigen, en of deze technieken in de toekomst bruikbaar zijn voor voedingsinterventie-studies. 


\section{Gaarne wil ik bedanken:}

- FOPPE TEN HOOR, die in 't begin de honneurs van promotor op zich heeft genomen.

- Mijn uiteindelijke promotor, Gerard Hornstra die naast suggesties voor het onderzoek, steeds maar weer de tijd vond om mijn ellenlange manuscripten door te lezen, en te voorzien van commentaar. Aangezien elke suggestie en elk commentaar weer een boel extra werk betekende, hoopte ik dat hij er wel eens genoeg van zou krijgen, maar zijn doorzettingsvermogen was nog groter dan dat van mij. Mede hierdoor is er een groot aantal proeven uitgevoerd dat het verschijnen van dit proefschrift mogelijk heeft gemaakt.

- In het bijzonder wil ik mijn begeleider, en co-promotor, RONALD MENSINK bedanken voor de continue en plezante begeleiding en samenwerking, en voor het corrigeren van de manuscripten. Opvallend was wel dat de frequentie van begeleiding toenam als er koekjes op onze kamer waren, maar een causale relatie hierbij is niet wetenschappelijk onderzocht.

- Ook wil ik alle andere co-auteurs bedanken, niet alleen voor hun bijdragen aan het tot stand komen van de manuscripten, maar ook voor hun hulp en samenwerking bij de proeven:

FRED BROUNS, die het mogelijk heeft gemaakt om financiēle ondersteuning van de Isostar Sport Nutrition Foundation te krijgen voor de studie naar de effecten van inspanning en visolie suppletie (Hoofdstuk 3), en die ruimte en apparatuur ter beschikkking heeft gesteld om de proeven uit te voeren.

TJEERD DE VRIES, voor het begeleiden van alle inspanningsproeven (Hoofdstuk 3) en het uitvoeren van een deel van de analyses;

MAX HARDEMAN EN ZIJN COLLEGA's, voor het bepalen van de flexibiliteit van rode bloedcellen en de viscositeit van plasma-monsters voor die studie;

MONIQUE AL en RIAN VAN HOUWELINGEN voor het beschikbaar stellen van de monsters om de antioxydant status bij zwangeren en vrouwen met zwangerschapshypertensie te onderzoeken (Hoofdstuk 4 en 5).

DR. FRITS BÃR, die verantwoordelijk was voor de PTCA-procedure (Hoofdstuk 6). De samenwerking met hem maakte het mogelijk dat ik lipidenperoxydatie tijdens dotteren kon bestuderen. 
- I have very much appreciated the time and effort PROF. J.C.S. KLEINJANS, PROF. C.E. BLANCO, DR. F. BROUNS, PROF. A.T. DIPLOCK, and PROF. F.A. HUF invested in critically evaluating my thesis.

- Peggy KaKerissa wil ik bedanken voor de hulp bij het opzetten van de versnelde methode voor de isolatie van LDL, en JOGCHUM PLAT voor het additionele onderzoek naar de effecten van visolie-suppletie op de oxydatie van LDL in vitro.

- Natuurlijk wil ik ook alle mEDEWERKERS VAN DE VAKGROEP HUMANE BIOLOGIE bedanken; vooral de nuttige informatie en spontane assistentie van het, door mij zeer gewaardeerde, OBP was onmisbaar. Ook al die medewerkers, en toevallige voorbijgangers, die steeds maar weer verzocht werd om bloed af te staan voor allerlei testjes, wil ik van harte bedanken (NB: onder het mom 'eens gegeven, blijt gegeven', is retour-zending niet mogelijk).

- In het bijzonder wil ik mijn kamergenoten LIESBETH en TANJA bedanken dat ze het zonder al te veel klagen, en mogelijk tegen hun zin want ze zaten altijd met de rug naar mij toe, toch met mij hebben kunnen uithouden.

- Verder wil ik HASIBE en FRANK bedanken voor het uitzoeken van monsters en voor het uitvoeren van analyses.

- Ik wil ook alle vakgroepen bedanken die mij, al dan niet met hun medeweten, chemicaliēn en laboratorium materialen hebben geleend. Vooral zonder de apparatuur die IRENE en ELS (vakgroep Gezondheidsrisico-analyse en Toxicologie) mij ter beschikking hebben gesteld zou het onderzoek beschreven in dit proefschrift niet binnen een decennium gereed zijn geweest. Verder wil ik de medewerkers van de technische dienst, met name MAURICE HUINCK, bedanken voor de snelle hulp die zij boden bij technische problemen.

- Ook was ik zeer verheugd over de spontane medewerking die veel bedrijven wilden verlenen bij het tot stand komen van de onderzoeken beschreven in dit proefschrift. In het bijzonder wil ik de FIRMA ORTHICA uit Weesp bedanken voor het gratis beschikbaar stellen van visolie-capsules.

In addition, I would like to thank F. HOFFMANN-LA ROCHE (Basel, Switzerland) for the free supply of vitamin $E$ capsules, and for their help and support during the development of the assay for antioxidant analysis in plasma.

- MARCEl \& NORA, bedankt voor jullie steun, i.c. koffie, bier, geduld, en gezelligheid.

- Als laatste wil ik JOS, DAPHNE en TruUs (samen met mij ook vaak aangeduid als 'De Feestcommissie') bedanken voor het systematisch (of dwangmatig) aanwezig zijn bij de maandelijkse sociale bijeenkomsten in Charlemagne. Het was alleen vervelend dat we deze bijeenkomsten zo vaak moesten onderbreken om te gaan werken. 


\section{CURRICULUM VITAE}

Gerard Simon Oostenbrug was bom in Deventer (The Netherlands), on February 18th, 1962. After graduating from secondary school in 1981, and military service, he completed a higher education for medical laboratory analyst specialized in clinical chemistry in Nijmegen, The Netherlands, in 1987. He fulfilled his practical period at the Department of Clinical Chemistry and Hematology of the Canisius-Wilhelmina Hospital in the same city. Immediately thereafter, he started his academic education at the University of Limburg in Maastricht (since September 1996: Maastricht University), The Netherlands, where he studied Biological Health Science at the Faculty of Health Sciences. In addition, he completed a statutory course to perform animal studies. After a practical period at the Department of Human Biology of the University of Limburg, during which he studied the effects of dietary fatty acids on serum cholesterol levels in Syrian hamsters, Gerard graduated in 1991, and started the Ph.D. research described in this thesis at the same department.

Gerard Simon Oostenbrug werd geboren op 18 februari 1962 te Deventer. In 1981 behaalde hij het VWO-B diploma aan de Alexander Hegius Scholengemeenschap in die gemeente. Na militaire dienst, studeerde Gerard vanaf 1983 aan de Opleiding Laboratoriumpersoneeel Amhem-Nijmegen te Nijmegen. Gedurende het laatste half jaar liep Gerard stage op de afdeling Klinische-Chemie en Hematologie van het Canisius-Wilhelmina Ziekenhuis te Nijmegen, en hij behaalde in juni 1987 het HBOdiploma voor Klinisch-Chemisch analist. Hiema ging Gerard Gezondheidswetenschappen studeren aan de Rijksuniversiteit Limburg (sinds september 1996: Universiteit Maastricht), met de afstudeerrichting Biologische Gezondheidkunde. Hij behaalde tevens de erkenning als onderzoeker ex artikel 9 van de Wet op de dierproeven, en verrichtte onderzoek naar de effecten van vetzuren in de voeding op het plasma cholesterol gehalte in de hamster gedurende een stage bij de vakgroep Humane Biologie van die universiteit. In augustus 1991 ontving hij zijn doctoraal diploma, en werd aansluitend bij dezelfde vakgroep aangesteld als Assistent In Opleiding, alwaar hij wetenschappelijk onderzoek verrichtte zoals beschreven in dit proefschritt. 
The aim of the studies described in this thesis was to investigate parameters of lipid peroxidation and antioxidant status in situations in which the degree of oxidative stress and/or antioxidative protection is altered.

Increasing the availability of a major substrate for lipid peroxidation, polyunsaturated fatty acids (PUFA), by supplementing the diet with highly unsaturated fish oil concentrates, increased the in vitro oxidation of low density lipoproteins in a first study. However, in a second, larger, study this effect was not confirmed. During exhaustive exercise plasma antioxidant levels and the deformability of red blood cells decreased. Although this may indicate increased oxidative stress, additional parameters of lipid peroxidation have to be measured to substantiate this. A combination of fish oil supplementation and exercise did not augment the effects observed during pre-supplementation exercise. The large increase in fatty acids levels during pregnancy was accompanied by a similar increase in plasma tocopherols levels, which might prevent oxidative stress due to the increase in plasma unsaturation. However, plasma carotenoid and retinol levels did not increase, but their role as antioxidants is sometimes disputed. Mild pregnancy-induced hypertension (PIH) did not affect plasma antioxidant levels, although some levels appear to decrease from the third trimester to postpartum, as compared with normotensive pregnant women. Nonetheless, the plasma antioxidant levels of the neonate were not affected by mild PIH of the mother. Also, in patients undergoing percutaneous transluminal coronary angioplasty (PTCA), there was no clear evidence indicating increased lipid peroxidation after this medical procedure associated with the production of oxygen free radicals.

It is concluded that under the investigated conditions of potentially increased oxidative stress, little or inconsistent evidence for increased systemic lipid peroxidation was present. 